\title{
Paths, Crystals and Fermionic Formulae
}

\author{
G. Hatayama* A. Kuniba*, M. Okadoł T. Takagi ${ }^{\ddagger}$ and Z. Tsuboi*
}

Dedicated to Professor Barry McCoy on the occasion of his sixtieth birthday

\begin{abstract}
We introduce a fermionic formula associated with any quantum affine algebra $U_{q}\left(X_{N}^{(r)}\right)$. Guided by the interplay between corner transfer matrix and Bethe ansatz in solvable lattice models, we study several aspects related to representation theory, most crucially, the crystal basis theory. They include one dimensional sums over both finite and semi-infinite paths, spinon character formulae, Lepowski-Primc type conjectural formula for vacuum string functions, dilogarithm identities, $Q$-systems and their solution by characters of various classical subalgebras and so forth. The results expand [HKOTY1] including the twisted cases and more details on inhomogeneous paths consisting of non-perfect crystals. As a most intriguing example, certain inhomogeneous one dimensional sums conjecturally give rise to branching functions of an integrable $G_{2}^{(1)}$-module related to the embedding $G_{2}^{(1)} \hookrightarrow B_{3}^{(1)} \hookrightarrow D_{4}^{(1)}$.
\end{abstract}

\section{Introduction}

Fermionic formulae are certain $q$-polynomials or $q$-series expressed as a specific sum of products of $q$-binomial coefficients. In this paper, which is an extended version of [HKOTY1, we introduce the fermionic formulae associated with any quantum affine algebra and discuss several aspects related to integrable systems and representation theory. For twisted cases, they are new even at $q=1$. Before going into the details, let us venture a brief historical account of the results scattering over immense literatures.

The first fermionic formula $(q=1)$ appeared in eq.(45) of Bethe's paper $\mathrm{Be}$ in 1931. It was given as the number of solutions to the Bethe equation for the Heisenberg spin chain based on the string hypothesis. Subsequent developments took place mainly from the 80 's. Here we divide them into the three main streams as arranged in Sections 1.1 1.3 below.

\footnotetext{
*Institute of Physics, University of Tokyo, Komaba, Tokyo 153-8902, Japan

${ }^{\dagger}$ Department of Informatics and Mathematical Science, Graduate School of Engineering Science, Osaka University, Toyonaka, Osaka 560-8531, Japan

${ }_{\ddagger}$ Department of Applied Physics, National Defense Academy, Yokosuka 239-8686, Japan
} 


\subsection{Quasi-particle picture}

Probably it was the Stony Brook group who first used the physics terminology fermionic for affine Lie algebra characters. Based on the Bethe ansatz analysis of integrable 3-state Potts chain [KM], Kedem and McCoy discovered a novel fermionic expression of the branching functions for the coset pair of the affine

Lie algebras $\left(A_{3}^{(1)}\right)_{1} \oplus\left(A_{3}^{(1)}\right)_{1} /\left(A_{3}^{(1)}\right)_{2}$. There was no minus sign in the sum as opposed to the well known Feigin-Fuchs-Rocha-Caridi bosonic formula for Virasoro characters. This outstanding feature was due to the fermionic nature of the lowlying excitations characterized by the roots of the Bethe type equation. Based on an elaborate analysis of the fermionic selection rule on the roots, they actually went beyond the characters of chiral blocks, and succeeded in constructing the modular invariant partition functions, i.e., bilinear combinations thereof, as a fermionic $q$-series.

Focusing on the constituent chiral blocks, Kedem et al. subsequently proposed the fermionic formulae for a variety of conformal field theory (CFT) models including the non-unitary Virasoro minimal ones KKMM1, KKMM2. Through these studies, the quasi-particle picture was put forward as a general concept of lowlying excitations leading to fermionic formulae [DKKMM], the Bailey pair technique was explored [BMSW] and the Rogers-Ramanujan type identities were interpreted as an equivalence of bosonic and fermionic descriptions KMM. We refer to [M] for the original survey.

The progress by the Stony Brook group inspired further developments from the viewpoint of Yangian symmetry and the "spinon basis" in integrable $A_{1}^{(1)}$ modules BPS, BLS1, BLS2. These ideas led to a proof of fermionic formulae by means of the chiral vertex operators and brought a new description of the CFT Hilbert spaces. We remark that most of the formulae in these works are level truncated (or restricted) in the sense explained in Section 1.3. See also [ANOT, NY2, NY3 for more aspects concerning the spinon interpretations.

In the early stage of these developments, main interests were directed to $q$-series rather than $q$-polynomials. The idea of "finitizing" the former to the latter came with [Me] as an ultraviolet cutoff in the quasi-particle picture. It was followed by many papers, most typically [Ber, $\mathrm{Wa}$, Sc, BMS], where the fermionic formulae were established for a polynomial analogue of $A_{1}^{(1)}$ coset Virasoro characters including the fractional level cases. See also [FW1, FW2]. An essential question in finitization is the meaning of the consequent $q$-polynomials. We postpone the discussion on this point to Section 1.3 .

\subsection{Combinatorics of Bethe ansatz}

The advances achieved in the 90's sketched in Section 1.1 may be viewed as a renaissance in the history of fermionic formulae linked up with CFT. Actually, fermionic formulae had been obtained in the middle 80's in a different context by Kerov, Kirillov and Reshetikhin [KKR, KR1] for the Kostka polynomial.

The Kostka polynomial is a $q$-analogue of the multiplicity of the irreducible $\mathfrak{s l}_{n}$-module $V_{\lambda}$ in the $m$-fold tensor product $V_{\left(\mu_{1}\right)} \otimes V_{\left(\mu_{2}\right)} \otimes \cdots \otimes V_{\left(\mu_{m}\right)}$. Here 
for a partition $\lambda=\left(\lambda_{1}, \cdots, \lambda_{n}\right)\left(\lambda_{1} \geq \cdots \geq \lambda_{n} \geq 0\right) V_{\lambda}$ stands for the irreducible $\mathfrak{s l}_{n}$-module with highest weight $\sum_{i=1}^{n-1}\left(\lambda_{i}-\lambda_{i+1}\right) \Lambda_{i}$ with $\Lambda_{i}$ being the fundamental weights of $\mathfrak{s l}_{n}$. In particular, $V_{\left(\mu_{i}\right)}$ is the symmetric tensor representation of degree $\mu_{i}$. The Kostka polynomial plays an important role in algebraic combinatorics, and is generalized to the cases where the components of the tensor product are not necessarily symmetric tensor representations. See e.g. [LLT, LT, S, SW].

As the Stony Brook group, the approach by Kerov et al. was also based on the Bethe ansatz1, but it had several distinctive features. Postulating the completeness of string hypothesis, they derived the $q=1$ fermionic formula as the multiplicity of irreducible $A_{n}$-modules in the tensor product of finitedimensional Yangian $Y\left(A_{n}\right)$-modules. Their formula admitted a natural $q$ analogue matching the Kostka polynomial. In this context, they treated $q$ polynomials without embarking on $q$-series and finitization. Later, Kirillov and Reshetikhin pushed these ideas further to extend the $q=1$ fermionic formula from $A_{n}$ to arbitrary classical simple Lie algebra $X_{n}$ [KR2]. There was no level truncation in those formulae ("classically restricted" in our terminology), and passage to the infinite series and connection to $A_{n}^{(1)}$ branching functions came afterward in 1995 by Kirillov as his Conjecture 4 in [Ki4].

The studies [Ki1, Ki2, KR2] on the completeness of the string hypothesis are relevant to polynomial fermionic formula at $q=1$ with no level truncation. In this sense they are most natural continuation of Bethe's original treatise. The invention of rigged configurations, bijection to semistandard Young tableaux [KKR, KR1] and the Kirillov-Reshetikhin conjecture [KR2] (see also Conjecture 2.1) was one of the earliest applications of the Bethe ansatz to combinatorics and representation theory.

When inferring the $q$-analogue at the level of fermionic formulae, the Kostka polynomial was the crucial guide for the $A_{n}$ case. To seek the clue for the other cases is a natural question which was also raised in the end of Section 1.1. More broadly, one may ask; what is the meaning, definition or characterization of the $q$-polynomial that should be represented by a fermionic formula?

\subsection{Corner transfer matrix and crystals}

The answer to the question had been prepared in Baxter's corner transfer matrix (CTM) method [B], which is another fundamental tool in solvable lattice models. The method introduces the quantity called one dimensional sums (1dsums). They are $q$-polynomials growing with lattice size, which eventually tend to affine Lie algebra characters in the infinite lattice limit. Such a phenomenon was first worked out for the celebrated ABF model ABF, FB and subsequently for other restricted solid-on-solid (RSOS) DJKMO1, JMO as well as vertex models DJKMO2]. In these works in the 80's, identification of 1dsums with characters was done mainly through explicit formulae (mostly bosonic one), therefore it was hard to go much beyond tractable examples.

\footnotetext{
${ }^{1}$ Their Bethe equation is different from the one in $\mathrm{KM}$.
} 
The advent of Kashiwara's crystal basis theory [Ka1] and its applications to vertex KMN1] and RSOS models [JMMO, DJO swept the difficulty away. The theory provided an intrinsic definition of $1 \mathrm{dsums}$ as well as a conceptual proof that their infinite lattice limit must be affine Lie algebra characters. The foregoing curiosity on 1dsums was resolved by the fundamental theorem [KMN1]: crystals of integrable highest weight modules are isomorphic to semi-infinite tensor products of finite crystals. This propaganda is simply called path realization.

The crystal basis theory is a theory of quantum groups at $q=0$ having a source in CTM with numerous applications. Here we just mention two examples relevant to this paper. The first one is the proof of the Kirillov conjecture on branching functions by Nakayashiki and Yamada [NY1. It was done by introducing the classically restricted $1 \mathrm{dsums}$ as an intrinsic characterization of the Kostka polynomial in terms of crystals. They are intermediate ones between the level restricted ones for RSOS and the unrestricted ones for vertex models, and will be essential to our discussion on fermionic formulae without level truncation. The second example is the study of Demazure crystals. Recall that Demazure modules are certain finite-dimensional subspaces of integrable highest weight modules labeled by Weyl group elements. Demazure modules at $q=0$ are called Demazure crystals [Ka2]. It was shown [KMOTU] that under the path realization, the restriction of 1 dsums to a certain Demazure crystal agrees with the finitization in the CTM language. See Table 1 in KMOTU for a summary of distinctive features of the three kinds of paths; unrestricted, classically restricted and (level) restricted ones. Our fermionic formulae $M_{\infty}$ and $M_{l}$ in this paper correspond to the latter two, respectively.

\subsection{Physical combinatorics}

Throughout the story so far, it should be emphasized that the two essential ingredients in solvable lattice models, Bethe ansatz and CTM, play complementary roles. The CTM and crystal theory provide an intrinsic definition of 1 dsums $X$ and characterization as branching coefficients of integrable highest weight modules ( $q$-series case) or Demazure modules (finitized $q$-polynomial case). On the other hand, the Bethe ansatz offers a specific formula $M$ which is fermionic. Therefore a synthesis of the two ideas reads

$$
X=M \text {. }
$$

To prove and understand this equality in the maximal generality is a fundamental problem in "Physical Combinatorics" $[$ in the beginning of the 21st century.

In HKOTY1 we have launched a fermionic formula $M$ for general nontwisted affine Lie algebras such that the above identity conjecturally holds. It is a $q$-analogue of [KR2], which covers a large list of known fermionic formulae under suitable specializations. In this paper we extend our proposal including the twisted cases where none was known even at $q=1$. Our generalization is not only in the direction of algebras. The earlier studies on 1dsums

\footnotetext{
${ }^{2}$ Named by Jean-Yves Thibon.
} 
[KMN1, KMOTU] were done exclusively for perfect crystals and homogeneous paths (cf. Section 1.5). Thus it remains a challenge to characterize the infinite lattice limit of 1dsums over inhomogeneous paths consisting of not necessarily perfect crystals. This is also a main subject of the paper. We propose a curious answer compatible with the thermodynamic Bethe ansatz as well as the crystal theoretic scheme in HKKOT. Roughly, inhomogeneity affects the path realization into that for tensor products of several highest weight modules. Non-perfectness leads to a description in terms of embeddings of affine Lie algebras. See AM, DMN, HKMW for a physical background of considering inhomogeneous paths.

Before going into the contents of the paper, we note further aspects of the subject including some latest ones; applications to quasi-hypergeometric functions [A], exclusion statistics [BM, BS2], decomposition of the KirillovReshetikhin modules $W_{s}^{(k)}$ (see Section 2.3) into classical irreducible modules [C1], the Feigin-Stoyanovsky theory [FS], fermionic formulae for the spaces of coinvariants associated to $\widehat{s l}_{2}$ [FKLMM], modular representations of Hecke algebras [FLOTW], approaches by various bijections and tableaux $\mathrm{FOW}$, KSS, SW, S, SS1], a graphical computational algorithm of fermionic formulae $\llbracket \mathrm{K} \|$, dilogarithm identities [Ki4], fermionic formulae for weight multiplicities KN], connection to geometry of quiver varieties [L], N], spinons at $q=0$ [NY3, NY4, fermionic formulae from fixed boundary RSOS models [OPW], decomposition of level 1 modules by level 0 actions [Ta, KKN, BS1, bosonic formulae from crystals [SS2, KMOTU], etc.

\subsection{Contents of the paper}

Let us explain the contents of the paper in detail along Sections 2 6 6 and Appendices A-D.

In Section 2, we fix basic notations on affine Lie algebra $\mathfrak{g}=X_{N}^{(r)}$ following [Kad, and crystals of $U_{q}(\mathfrak{g})\left(\right.$ or $\left.U_{q}^{\prime}(\mathfrak{g})\right)$ ] following [KMN1, KMN2]. We consider two categories of representations and crystals; one for the integrable highest weight modules and the other for finite-dimensional modules. Crystals in the latter category are called finite crystals, for which the notion of perfect crystals is explained. We introduce irreducible finite-dimensional $U_{q}^{\prime}(\mathfrak{g})$-modules $W_{s}^{(k)}$ and associated conjectural family of finite crystal basis $B^{k, s}$. In particular, all the $B^{k, s}$ 's are conjectured to be perfect in the twisted cases $r>1$.

In Section 3 we define a path as an element of the semi-infinite tensor product $\cdots \otimes B \otimes B$ of a finite crystal $B$ obeying a certain boundary condition. Here $B$ has the form $B=B^{k_{1}, s_{1}} \otimes B^{k_{2}, s_{2}} \otimes \cdots \otimes B^{k_{d}, s_{d}}$ and the resulting paths are called inhomogeneous in the sense that $\left(k_{i}, s_{i}\right)$ can be $i$-dependent in general. Homogeneous paths $(d=1)$ consisting of perfect $B=B^{k_{1}, s_{1}}$ were originally studied in KMN1. We state Conjectures 3.3 and 3.6, which identify the set of paths with the tensor product of crystals of integrable highest

\footnotetext{
${ }^{3}$ Although denoted by the same letter, the deformation parameter $q$ is unrelated with the argument of fermionic formulae having the meaning $q=e^{-\delta}$ in terms of the null root $\delta$.
} 
weight modules. The former conjecture concerns the situation where all $B^{k_{i}, s_{i}}$ 's are perfect. It can actually be proved for a large list of crystals as mentioned in Remark 3.5. The latter conjecture deals with non-perfect cases, and is described, rather curiously, in terms of the embedding of the affine Lie algebras $B_{n}^{(1)} \hookrightarrow D_{n+1}^{(1)}, C_{n}^{(1)} \hookrightarrow A_{2 n-1}^{(1)}, F_{4}^{(1)} \hookrightarrow E_{6}^{(1)}$ and $G_{2}^{(1)} \hookrightarrow B_{3}^{(1)} \hookrightarrow D_{4}^{(1)}$. See (3.5). It originates in the thermodynamic Bethe ansatz calculation of RSOS central charges [Ku] and is supported by computer experiments. In Section 3.3 we introduce one dimensional sums (1dsums) $X_{l}(B, \lambda, q)$ in (3.9), where $l \in \mathbb{Z}_{\geq 0}$ or $l=\infty$ is the level of restriction and $\lambda$ is the weight of the relevant paths. They are crystal theoretic formulation of a trace of corner transfer matrices going back to ABF, B]. The 1dsums over inhomogeneous paths firstly appeared in [NY1] for $A_{n}^{(1)}$ case. It makes essential use of the combinatorial $R$ matrices, i.e., the isomorphism and energy functions on tensor products of finite crystals. Our definition basically follows theirs except a subtle "boundary energy" (2nd term in (3.8)) which becomes crucial in general if $\mathfrak{g} \neq A_{n}^{(1)}$. Our fundamental observation is Conjecture 3.10 equating the 1 dsums on finite tensor products with fermionic formulae. In Section 3.4 we explain how the finite tensor products are embedded into the crystals of integrable highest weight modules.

In Section 1 we define fermionic formulae labeled by the tensor product $W=W_{s_{1}}^{\left(k_{1}\right)} \otimes \cdots \otimes W_{s_{d}}^{\left(k_{d}\right)}$. There are two versions denoted by $M_{l}(W, \lambda, q)(4.5)$ and $\tilde{M}_{l}(W, \lambda, q)(4.20)$. Compared to $M_{l}, \tilde{M}_{l}$ involves unphysical contributions from negative vacancy numbers in the Bethe ansatz. However as emphasized in HKOTY1, they conjecturally coincide and the latter enjoys a skew invariance under the Weyl group action (Conjecture 4.3). We present the spinon character formulae derived in the infinite tensor product limit in Propositions 4.5 and 4.6. Proposition 4.10 describes the other limit where $q$ is replaced with $q^{-1}$.

In Section 5 we present a conjectural $q$-series formula $N_{l}(\lambda, q)$ for level $l$ vacuum string functions $[\mathrm{Kad}$. It extends the nontwisted cases [KNS], where a Bethe ansatz interpretation has been proposed. We point out the relation (5.4) to the fermionic formula $M_{l}$, although its meaning is yet to be clarified. So far the conjecture is known valid for $A_{1}^{(1)}\left[\mathrm{LP}\right.$ and $A_{n}^{(1)}[\mathrm{G}$, HKKOTY as well as $l=1$ case $\mathrm{Kad}$. The $q$-series exhibits an asymptotic behavior consistent with [Kad under the dilogarithm sum rule (6.4). In particular the sum rule for the twisted cases $X_{N}^{(r)}(r>1)$ can be obtained from the nontwisted one $X_{N}^{(1)}$ by a simple unfolding procedure due to (6.5). We remark that a generalization of $N_{l}(\lambda, q)$ related to tensor product of several vacuum modules has been proved for $A_{n}^{(1)}$ and conjectured for the other nontwisted cases in the section 6.1 of [HKKOTY].

Section 6 is devoted to the $Q$-system. It is a possible character identity among $Q_{j}^{(a)}=\operatorname{ch~} W_{j}^{(a)}$ having a form of $2 \mathrm{~d}$ Toda equations and essentially governs the fermionic formulae at $q=1$. It was proposed firstly in KR2, Ki3] for nontwisted cases. We introduce the twisted cases $X_{N}^{(r)}$ by folding the $Q$-system of the underlying simply laced case $X_{N}^{(1)}$ by an order $r$ Dynkin diagram automorphism. Explicit solutions are presented for $A_{2 n}^{(2)}, A_{2 n-1}^{(2)}, D_{n+1}^{(2)}$ and $D_{4}^{(3)}$ in terms 
of characters of various classical subalgebras. The results given there, Appendix G and [KR2, HKOTY1], cover all the non-exceptional affine Lie algebras $\mathfrak{g}$, and exhaust the possible choices of their classical simple Lie subalgebras obtained by removing a vertex in the Dynkin diagrams. We find that only the choice of the classical subalgebra $\stackrel{\circ}{\mathfrak{g}}$ (Table 2) fits the requirements $(\mathrm{A})$ and $(\mathrm{C})$ of Theorem 6.3. The identity like (6.14) therein is sometimes called "combinatorial completeness of the string hypothesis in the Bethe ansatz". For nontwisted cases $U_{q}\left(X_{N}^{(1)}\right)$, the reason is that $M_{\infty}(W, \lambda, 1)$ naturally emerges from a formal counting of the number of solutions to the Bethe equation $\mathrm{OW}$ for rational vertex models with Yangian symmetry $Y\left(X_{N}\right)$ t. However for twisted cases, we have not succeeded in deriving our $q=1$ fermionic formula from the relevant Bethe equation [RW] under a string hypothesis. Consequently we do not yet know an interpretation of the identity (6.14) as a combinatorial completeness for the rational limit of trigonometric $U_{q}^{\prime}\left(X_{N}^{(r)}\right)$ vertex models. See the end of Section 6.3 for related remarks. In order to find the $q=1$ fermionic formula with $r>1$, we set out by postulating the $Q$-system first instead of investigating the Bethe equation. Once the $Q$-system is specified, one can execute parallel calculations with the section 8.2 of HKOTY1. Our $M_{\infty}(W, \lambda, 1)$ has come up from such analyses simultaneously with Theorem 6.3. To invent the $q$-analogue is then straightforward by presuming Conjecture 3.10, i.e., $M_{\infty}(W, \lambda, q)=q^{-D} X_{\infty}(B, \lambda, q)$ for some $D$.

Appendix A provides a list of the basic fermionic formulae $M_{\infty}\left(W_{s}^{(a)}, \lambda, q^{-1}\right)$ for twisted cases. The results are analytically obtained for non-exceptional algebras. (See appendix A of [HKOTY1 for a similar list for nontwisted cases.) Appendix B gives the explicit form of Proposition 4.10. Appendix $\mathrm{O}$ contains the solution of $B_{n}^{(1)} Q$-system in terms of $D_{n}$ characters. Appendix D provides the lists of isomorphism $B^{1, s_{1}} \otimes B^{1, s_{2}} \simeq B^{1, s_{2}} \otimes B^{1, s_{1}}\left(1 \leq s_{2} \leq s_{1} \leq 2\right)$ and energy function for $\mathfrak{g}=B_{3}^{(1)}, C_{2}^{(1)}, D_{4}^{(1)}, A_{3}^{(2)}, A_{4}^{(2)}, D_{3}^{(2)}$ and $D_{4}^{(3)}$. They form sufficient data to compute the inhomogeneous 1 dsums over the tensor products of these crystals.

Let us close the introduction with two remarks. Solvable RSOS models at a generic primitive root of unity give rise to yet further 1dsums associated with complicated energy functions. A typical example is [FB] corresponding to $A_{1}^{(1)}$, and as noted in Section 1.1, intricate arithmetic structures have been worked out on the fermionic formula and relation to fractional level representations [BMS, BMSW, FW2]. The crystal theory has not yet been adapted to such situations. It may be interesting to seek an analogous generalization of our fermionic formula.

According to Section 3.4, the limit of our 1 dsums $\lim _{L \rightarrow \infty} q^{-D} X_{l}\left(B^{\otimes L}, \lambda, q\right)$ ( $D$ : normalization) yields the $q$-series having an affine Lie algebraic meaning. Such phenomena correspond, though rather roughly, to the so called "regime III" or "antiferromagnetic regime" in the language of solvable RSOS models or

${ }^{4}$ Although it is not $M_{\infty}$ but $\tilde{M}_{\infty}$ that enters 6.14 ), we admit $M_{\infty}=\tilde{M}_{\infty}$ here according to Conjecture 4.3 . 
vertex models. However, those models indicate that the other regimes are no less interesting. In particular, the analysis of the opposite "regime II" or "ferromagnetic regime" renders the following question: what is the representation theoretical meaning of another $q$-series $\lim _{L \rightarrow \infty} q^{-D^{\prime}} X_{l}\left(B^{\otimes L}, \lambda, q^{-1}\right)\left(D^{\prime}:\right.$ another normalization)? Compared with "regime III", such issues in "regime II" are yet poorly understood See Proposition 4.10, Appendix B and (5.4). For $l=\infty$ the problem is relevant to another topic, the soliton cellular automata associated with crystal bases HKOTY2.

\section{Acknowledgements}

The authors thank V. Chari, T. Nakanishi, A. Schilling, M. Shimozono and J. Suzuki for useful discussions and Y. Yamada for the collaboration in their previous work [HKOTY1]. A.K. and M.O. are partially supported by Grant-in-Aid for Scientific Research from the Ministry of Education, Culture, Sports, Science and Technology of Japan. Z.T. is supported by JSPS Research Fellowships for Young Scientists.

\section{Conjectural family of crystals}

In this section we prepare necessary notations for the affine Lie algebra $\mathfrak{g}$, quantum affine algebra $U_{q}(\mathfrak{g})$ and its crystals. We also present a conjectural family of crystals revealed by the Bethe ansatz.

\subsection{Affine Lie algebra $X_{N}^{(r)}$}

Let $\mathfrak{g}$ be a Kac-Moody Lie algebra of affine type $X_{N}^{(r)}=A_{n}^{(1)}(n \geq 1), B_{n}^{(1)}(n \geq$ $3), C_{n}^{(1)}(n \geq 2), D_{n}^{(1)}(n \geq 4), E_{n}^{(1)}(n=6,7,8), F_{4}^{(1)}, G_{2}^{(1)}, A_{2 n}^{(2)}(n \geq 1), A_{2 n-1}^{(2)}(n \geq$ 2), $D_{n+1}^{(2)}(n \geq 2), E_{6}^{(2)}$ and $D_{4}^{(3)}$. As is well known, $X_{N}^{(r)}$ is realized as the canonical central extension of the loop algebra based on the pair $\left(X_{N}, \sigma\right)$ of finitedimensional simple Lie algebra $X_{N}$ and its Dynkin diagram automorphism $\sigma$ of order $r=1,2,3$. The vertices of the Dynkin diagram of $\mathfrak{g}=X_{N}^{(r)}$ are labeled as in Table 1, which is quoted from TABLE Aff1-3 in Kac.

Among finite-dimensional subalgebras of $\mathfrak{g}$, the following $\mathfrak{g}$ and $\mathfrak{g}_{0}$ will play an essential role. Here $\stackrel{\circ}{\mathfrak{g}}$ is obtained by removing the 0 -th vertex from the Dynkin diagram of $\mathfrak{g}$. See the section 6.3 of $\mathrm{Kac}$. On the other hand $\mathfrak{g}_{0}$ is the subalgebra of $X_{N}$ fixed by $\sigma$. See the section 8.3 of [Kad]. Note that $\stackrel{\circ}{\mathfrak{g}}=\mathfrak{g}_{\overline{0}}$ except $\mathfrak{g}=A_{2 n}^{(2)}$.

Table 1 fixes the enumeration of the simple roots of $\stackrel{\circ}{\mathfrak{g}}$ by the restriction to $\{1, \ldots, n\}$. As for $\mathfrak{g}_{\overline{0}}$ we naturally employ the same enumeration with $\stackrel{\circ}{\mathfrak{g}}$ when

${ }^{5}$ If $\mathfrak{g}=A_{n}^{(1)}$ and $l<\infty$, the question can be answered indirectly through the level-rank duality. 
Table 1: Dynkin diagrams for $X_{N}^{(r)}$. The enumeration of the nodes with $I=$ $\{0,1, \ldots, n\}$ is specified under or the right side of the nodes. In addition, the numbers $t_{i}$ (resp. $t_{i}^{\vee}$ ) defined in (2.1) are attached above the nodes for $r=1$ (resp. $r>1$ ) if and only if $t_{i} \neq 1$ (resp. $t_{i}^{\vee} \neq 1$ ).

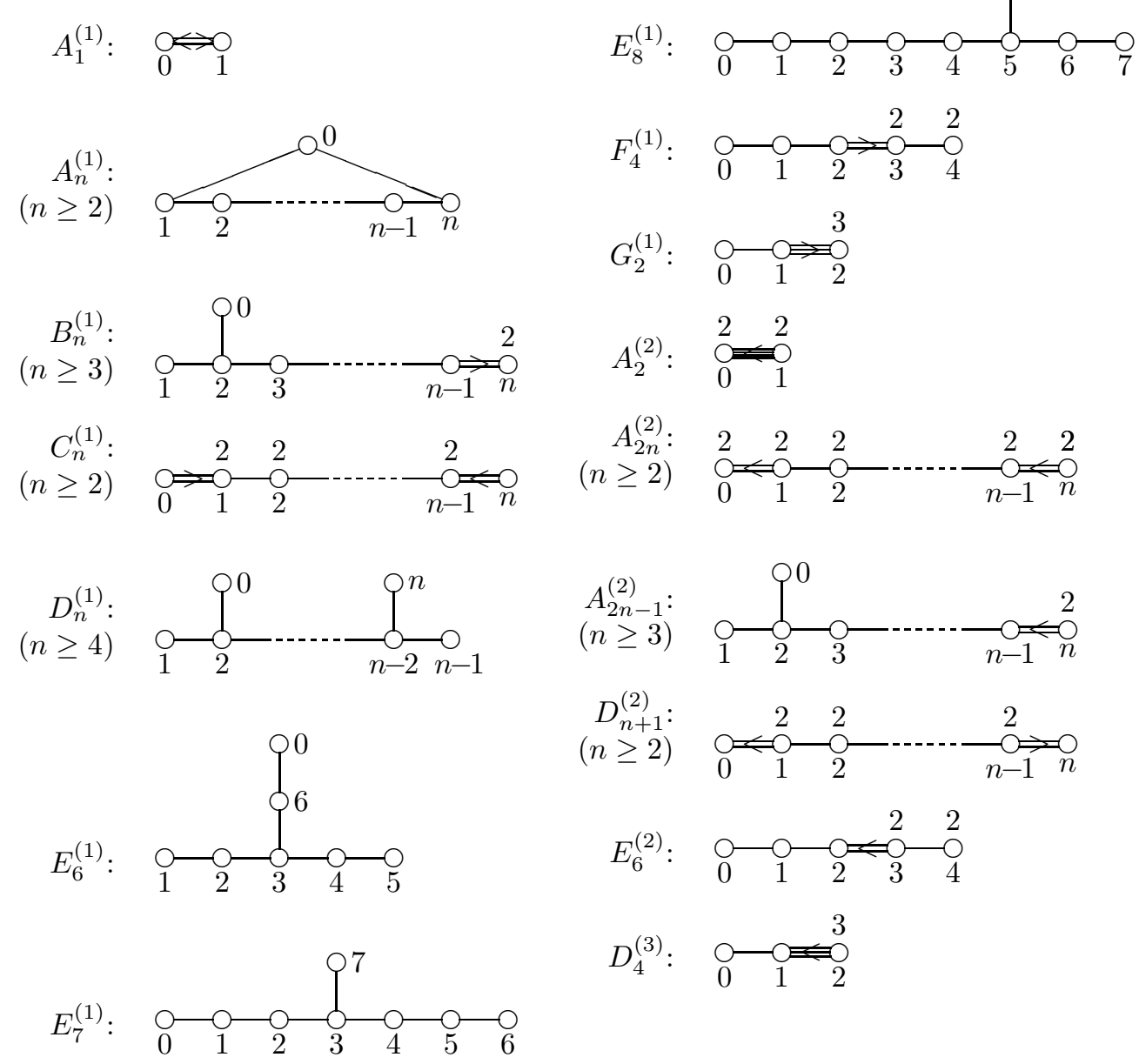


Table 2:

\begin{tabular}{c|cccccc}
$\mathfrak{g}$ & $X_{N}^{(1)}$ & $A_{2 n}^{(2)}$ & $A_{2 n-1}^{(2)}$ & $D_{n+1}^{(2)}$ & $E_{6}^{(2)}$ & $D_{4}^{(3)}$ \\
\hline$\stackrel{\mathfrak{g}}{ }$ & $X_{N}$ & $C_{n}$ & $C_{n}$ & $B_{n}$ & $F_{4}$ & $G_{2}$ \\
$\mathfrak{g}_{\overline{0}}$ & $X_{N}$ & $B_{n}$ & $C_{n}$ & $B_{n}$ & $F_{4}$ & $G_{2}$
\end{tabular}

$\mathfrak{g} \neq A_{2 n}^{(2)}$, since they are the same subalgebra. As for the conventions on $\mathfrak{g}_{\overline{0}}$ for $\mathfrak{g}=A_{2 n}^{(2)}$, see Section 4.1.

Let $\alpha_{i}, h_{i}, \Lambda_{i}(i \in I=\{0,1, \ldots, n\})$ be the simple roots, simple coroots, fundamental weights of $\mathfrak{g}$. Let $\delta$ and $c$ denote the generator of imaginary roots and the canonical central element, respectively. Recall that $\delta=\sum_{i \in I} a_{i} \alpha_{i}, c=$ $\sum_{i \in I} a_{i}^{\vee} h_{i}$ and the Kac label $a_{i}$ is given explicitly by

$$
\begin{aligned}
\delta & =\alpha_{0}+\cdots+\alpha_{n} & & \text { for } \mathfrak{g}=A_{n}^{(1)}, \\
& =\alpha_{0}+\alpha_{1}+2 \alpha_{2}+\cdots+2 \alpha_{n} & & \text { for } \mathfrak{g}=B_{n}^{(1)}, \\
& =\alpha_{0}+2 \alpha_{1}+\cdots+2 \alpha_{n-1}+\alpha_{n} & & \text { for } \mathfrak{g}=C_{n}^{(1)}, \\
& =\alpha_{0}+\alpha_{1}+2 \alpha_{2}+\cdots+2 \alpha_{n-2}+\alpha_{n-1}+\alpha_{n} & & \text { for } \mathfrak{g}=D_{n}^{(1)} \\
& =\alpha_{0}+\alpha_{1}+2 \alpha_{2}+3 \alpha_{3}+2 \alpha_{4}+\alpha_{5}+2 \alpha_{6} & & \text { for } \mathfrak{g}=E_{6}^{(1)} \\
& =\alpha_{0}+2 \alpha_{1}+3 \alpha_{2}+4 \alpha_{3}+3 \alpha_{4}+2 \alpha_{5}+\alpha_{6}+2 \alpha_{7} & & \text { for } \mathfrak{g}=E_{7}^{(1)} \\
& =\alpha_{0}+2 \alpha_{1}+3 \alpha_{2}+4 \alpha_{3}+5 \alpha_{4}+6 \alpha_{5}+4 \alpha_{6}+2 \alpha_{7}+3 \alpha_{8} & & \text { for } \mathfrak{g}=E_{8}^{(1)} \\
& =\alpha_{0}+2 \alpha_{1}+3 \alpha_{2}+4 \alpha_{3}+2 \alpha_{4} & & \text { for } \mathfrak{g}=F_{4}^{(1)} \\
& =\alpha_{0}+2 \alpha_{1}+3 \alpha_{2} & & \text { for } \mathfrak{g}=G_{2}^{(1)} \\
& =2 \alpha_{0}+2 \alpha_{1}+\cdots+2 \alpha_{n-1}+\alpha_{n} & & \text { for } \mathfrak{g}=A_{2 n}^{(2)} \\
& =\alpha_{0}+\alpha_{1}+2 \alpha_{2}+\cdots+2 \alpha_{n-1}+\alpha_{n} & & \text { for } \mathfrak{g}=A_{2 n-1}^{(2)}, \\
& =\alpha_{0}+\alpha_{1}+\cdots+\alpha_{n-1}+\alpha_{n} & & \text { for } \mathfrak{g}=D_{n+1}^{(2)}, \\
& =\alpha_{0}+2 \alpha_{1}+3 \alpha_{2}+2 \alpha_{3}+\alpha_{4} & & \text { for } \mathfrak{g}=E_{6}^{(2)} \\
& =\alpha_{0}+2 \alpha_{1}+\alpha_{2} & & \text { for } \mathfrak{g}=D_{4}^{(3)} .
\end{aligned}
$$

The dual Kac label $a_{i}^{\vee}$ is equal to $a_{i}$ of $\mathfrak{g}^{\vee}$ corresponding to the transposed Cartan matrix. We let $h^{\vee}=\sum_{i \in I} a_{i}^{\vee}$ denote the dual Coxeter number. Note that $h^{\vee}$ for $X_{N}^{(r)}$ is independent of $r$ (Remark 6.1 in $\mathrm{Kac}$ ).

For $i \in I$ we set

$$
t_{i}=\max \left(\frac{a_{i}}{a_{i}^{\vee}}, a_{0}^{\vee}\right), \quad t_{i}^{\vee}=\max \left(\frac{a_{i}^{\vee}}{a_{i}}, a_{0}\right) .
$$

They are given in Table 1. By the definition $t_{i}, t_{i}^{\vee} \in\{1,2,3\}$, and $\left(t_{i}, t_{i}^{\vee}\right)$ for $\mathfrak{g}$ is equal to $\left(t_{i}^{\vee}, t_{i}\right)$ for $\mathfrak{g}^{\vee}$. In the sequel, $t_{i}^{\vee}$ and $t_{i}$ are used only for $i \in I \backslash\{0\}$. For $1 \leq a \leq n$ we have

$$
t_{a}^{\vee}=1 \quad \text { if } r=1, \quad t_{a}=1 \quad \text { if } r>1,
$$


and especially for $\mathfrak{g}=A_{2 n}^{(2)},\left(t_{a}, t_{a}^{\vee}\right)=(1,2)$ for all $1 \leq a \leq n$. We remark that

$$
r a_{0} \sum_{a=1}^{n} \frac{1}{t_{a}^{\vee}}=N \quad \text { for any } X_{N}^{(r)} \text {. }
$$

Let $P=\bigoplus_{i \in I} \mathbb{Z} \Lambda_{i} \bigoplus \mathbb{Z} \delta$ be the weight lattice. We define the following subsets of $P: P^{+}=\sum_{i \in I} \mathbb{Z}_{\geq 0} \Lambda_{i}, P_{l}^{+}=\left\{\lambda \in P^{+} \mid\langle\lambda, c\rangle=l\right\}, \bar{P}=\sum_{i=1}^{n} \mathbb{Z} \bar{\Lambda}_{i}$, $\bar{P}^{+}=\sum_{i=1}^{n} \mathbb{Z}_{\geq 0} \bar{\Lambda}_{i}$. Here $\bar{\Lambda}_{i}=\Lambda_{i}-\left\langle\Lambda_{i}, c\right\rangle \Lambda_{0}$ is the classical part of $\Lambda_{i}$. This map is extended to a map on $P$ so that it is $\mathbb{Z}$-linear and $\bar{\delta}=0$. We also consider the classical weight lattice $P_{c l}=P / \mathbb{Z} \delta$. In this paper we canonically identify $P_{c l}$ with $\bigoplus_{i \in I} \mathbb{Z} \Lambda_{i} \subset P$. (See the section 3.1 of KMN1 for a precise treatment.) We let $\bar{Q}=\bigoplus_{i=1}^{n} \mathbb{Z} \alpha_{i}$ denote the classical root lattice. Let (.|.) be the standard bilinear form on $P$ normalized by $(\delta \mid \lambda)=\langle c, \lambda\rangle$ for any $\lambda \in P$. This normalization agrees with that in $\mathrm{Kac}$.

\subsection{Quantum affine algebra and crystals}

Let $U_{q}(\mathfrak{g})$ be the quantum affine algebra associated to $\mathfrak{g}$. We follow the section 2.1 of KKMN1 for its definitions. $U_{q}(\mathfrak{g})$ turns out a subalgebra of $U_{q}(\mathfrak{g})$ in an obvious manner. $U_{q}^{\prime}(\mathfrak{g})$ is the one without the generator $q^{d}$, where $d$ is the degree operator in $\mathfrak{g}$.

We next review terminology for crystals. A crystal basis $B$ of a $U_{q}(\mathfrak{g})$ $\left(U_{q}^{\prime}(\mathfrak{g}), U_{q}(\mathfrak{g})\right)$-module can be regarded as a set of basis vectors of the module at $q=0$. On $B$ one has the action of modified Chevalley generators (Kashiwara operators)

$$
\tilde{e}_{i}, \tilde{f}_{i}: B \longrightarrow B \sqcup\{0\} .
$$

For $b \in B$ we set $\varepsilon_{i}(b)=\max \left\{k \geq 0 \mid \tilde{e}_{i}^{k} b \neq 0\right\}, \varphi_{i}(b)=\max \left\{k \geq 0 \mid \tilde{f}_{i}^{k} b \neq 0\right\}$. If $B_{1}$ and $B_{2}$ are crystals, the crystal structure on the tensor product $B_{1} \otimes B_{2}$ is given by

$$
\begin{aligned}
& \tilde{e}_{i}\left(b_{1} \otimes b_{2}\right)= \begin{cases}\tilde{e}_{i} b_{1} \otimes b_{2} & \text { if } \varphi_{i}\left(b_{1}\right) \geq \varepsilon_{i}\left(b_{2}\right), \\
b_{1} \otimes \tilde{e}_{i} b_{2} & \text { otherwise, }\end{cases} \\
& \tilde{f}_{i}\left(b_{1} \otimes b_{2}\right)= \begin{cases}\tilde{f}_{i} b_{1} \otimes b_{2} & \text { if } \varphi_{i}\left(b_{1}\right)>\varepsilon_{i}\left(b_{2}\right), \\
b_{1} \otimes \tilde{f}_{i} b_{2} & \text { otherwise. }\end{cases}
\end{aligned}
$$

We mainly use two categories of crystals. The first one contains the crystal basis $B(\lambda)$ of the irreducible integrable $U_{q}(\mathfrak{g})$-module $L(\lambda)$ with highest weight $\lambda \in P^{+} . B(\lambda)$ is a $P$-weighted crystal. The other one contains a crystal basis $B$ of a finite-dimensional $U_{q}^{\prime}(\mathfrak{g})$-module. As opposed to $B(\lambda), B$ is a finite set and $P_{c l}$-weighted. We shall call it a finite crystal. (See HKKOT for the precise definition.) For a finite crystal $B$, we set $\varepsilon(b)=\sum_{i} \varepsilon_{i}(b) \Lambda_{i}, \varphi(b)=\sum_{i} \varphi_{i}(b) \Lambda_{i}$, wt $b=\varphi(b)-\varepsilon(b)$, and introduce the level of $B$ by

$$
\operatorname{lev} B=\min \{\langle c, \varepsilon(b)\rangle \mid b \in B\} .
$$


We further set $B_{\min }=\{b \in B \mid\langle c, \varepsilon(b)\rangle=\operatorname{lev} B\}$, and call an element of $B_{\min }$ minimal. Let $B_{1}, B_{2}$ be finite crystals of level $l_{1}, l_{2}$. Then $B_{1} \otimes B_{2}$ turns out a finite crystal of level $\max \left(l_{1}, l_{2}\right)$. A $U_{q}^{\prime}(\mathfrak{g})$-module can be viewed as a $U_{q}(\mathfrak{g})$-module. For the latter the irreducible representation with highest weight $\lambda \in \bar{P}^{+}$will be denoted by $V(\lambda)$.

We also need the notion of perfect crystals [KMN1, KMN2]. A perfect crystal is a finite crystal with an additional condition. Let $B$ be a finite crystal of level $l$. $B$ is said to be perfect if the maps $\varepsilon, \varphi: B_{\min } \longrightarrow P_{l}^{+}$are bijections. Set $\sigma=\varepsilon \circ \varphi^{-1}$. $\sigma^{6}$ is related to a Dynkin diagram automorphism and can be extended on $P^{+}$.

\subsection{Family $W_{s}^{(k)}$}

It is known CP1, CP2 that the irreducible finite-dimensional $U_{q}^{\prime}(\mathfrak{g})$-modules are characterized by $n$-tuple of polynomials $\left\{P_{a}(u)\right\}_{a=1}^{n}$ called Drinfel'd polynomials. Set $\hat{q}_{k}=q^{\left(\alpha_{k} \mid \alpha_{k}\right) / 2}$. We define an irreducible finite-dimensional $U_{q}^{\prime}(\mathfrak{g})$ module $W_{s}^{(k)}(1 \leq k \leq n, s \geq 1)$ by its Drinfel'd polynomials?

$$
P_{a}(u)= \begin{cases}\left(1-\hat{q}_{k}^{s-1} u\right)\left(1-\hat{q}_{k}^{s-3} u\right) \cdots\left(1-\hat{q}_{k}^{-s+1} u\right) & (a=k), \\ 1 & (a \neq k) .\end{cases}
$$

It is called a Kirillov-Reshetikhin module.

Conjecture 2.1. The finite-dimensional $U_{q}^{\prime}(\mathfrak{g})$-module $W_{s}^{(k)}$ has the following features:

(1) $W_{s}^{(k)}$ has a crystal basis $B^{k, s} . B^{k, s}$ is a finite crystal of level $\left\lceil\frac{s}{t_{k}}\right\rceil$. Moreover, it is perfect if $\frac{s}{t_{k}}$ is an integer, and not perfect if not an integer.

(2) As a $U_{q}(\mathfrak{g})$-module, $W_{s}^{(k)}$ decomposes itself into

$$
W_{s}^{(k)}=\bigoplus_{\lambda \in \bar{P}^{+}} M_{\infty}\left(W_{s}^{(k)}, \lambda, q=1\right) V(\lambda)
$$

where $M_{l}\left(W_{s}^{(k)}, \lambda, q\right)$ is defined in (4.5).

(3) Set $Q_{s}^{(k)}=\operatorname{ch} W_{s}^{(k)}$, then $Q_{s}^{(k)}$ satisfies the Q-system (6.1).

From (4.5) the decomposition (2) of $W_{s}^{(k)}$ has the form

$$
W_{s}^{(k)}=V\left(s \bar{\Lambda}_{k}\right) \oplus \cdots,
$$

where $\cdots$ contains irreducible modules with highest weights strictly lower than $s \bar{\Lambda}_{k}$ only. A list of such decompositions is available in Appendix A (by setting $q=1)$.

Several remarks are in order.

${ }^{6}$ Distinguish this with $\sigma$ in $\left(X_{N}, \sigma\right)$ which appeared in Section 2.1. CP2.

7 In the twisted cases, we are afraid that our normalization might not agree with that in ${ }^{8}$ The symbol $\lceil x\rceil$ denotes the smallest integer not less than $x$. 
Remark 2.2. Although they did not specify the Drinfel'd polynomials, parts (2) and (3) (for nontwisted cases) are essentially the Kirillov-Reshetikhin conjecture [KR2. In the nontwisted cases, part (2) of the conjecture was solved recently [C1] for all cases if $\mathfrak{g}$ is classical and several cases if exceptional. See also [C2].

Remark 2.3. In Ka3] an irreducible finite-dimensional $U_{q}^{\prime}(\mathfrak{g})$-module $W\left(\varpi_{i}\right)$ (fundamental representation) having a crystal basis is constructed. It should agree with our $W_{1}^{(i)}$.

Remark 2.4. It is noted that $t_{k}>1$ occurs only when $\mathfrak{g}=B_{n}^{(1)}(k=n), C_{n}^{(1)}(k \neq$ $n), F_{4}^{(1)}(k=3,4), G_{2}^{(1)}(k=2)$. In particular, $t_{k}=1$ for any $k$, if $\mathfrak{g}$ is simply laced or of twisted type. In these cases any $B^{k, s}$ is conjectured to be perfect.

Remark 2.5. There is a notion of coherent family of perfect crystals KKM. We also conjecture that $\left\{B^{k, t_{k} l}\right\}_{l \geq 1}$ forms a coherent family of perfect crystals for any $k$.

Remark 2.6. For twisted cases the following crystals are known explicitly.

$$
\begin{array}{ll}
\text { KMN2 } & A_{2 n}^{(2)}: B^{1, s}, A_{2 n-1}^{(2)}: B^{1, s}, D_{n+1}^{(2)}: B^{1, s} B^{n, s}, \\
\text { JnMO } & A_{2 n}^{(2)}: B^{k, 1}, A_{2 n-1}^{(2)}: B^{k, 1} .
\end{array}
$$

See also [KKM] for the explicit crystal structure of $B^{1, s}$. In JnMO one should notice that the labeling of the Dynkin diagrams is opposite. See also HKOTY1 Remark 2.3 for nontwisted cases.

\section{Paths and one dimensional sums}

Here we define a set of paths $\mathcal{P}(\boldsymbol{p}, B)$ and the one dimensional sum $X_{l}(B, \lambda, q)$. We then present several conjectures related to them.

\subsection{Energy function and Yang-Baxter equation}

We review the energy function and Yang-Baxter equation for finite crystals. Let $B$ be a finite crystal. Introduce an indeterminate $z$ and set

$$
\operatorname{Aff}(B)=\left\{z^{\gamma} b \mid \gamma \in \mathbb{Z}, b \in B\right\} .
$$

It is called the affinization of $B$. On $\operatorname{Aff}(B)$ one can define the action of $\tilde{e}_{i}$ and $\tilde{f}_{i}$ by

$$
\tilde{e}_{i}\left(z^{\gamma} b\right)=z^{\gamma+\delta_{i 0}}\left(\tilde{e}_{i} b\right), \quad \tilde{f}_{i}\left(z^{\gamma} b\right)=z^{\gamma-\delta_{i 0}}\left(\tilde{f}_{i} b\right) .
$$

Let $B_{1}$ and $B_{2}$ be finite crystals. Then there exists an isomorphism of crystals called the combinatorial $R$ [KMN1]:

$$
\begin{aligned}
R: \quad \operatorname{Aff}\left(B_{1}\right) \otimes A f f\left(B_{2}\right) & \longrightarrow A f f\left(B_{2}\right) \otimes A f f\left(B_{1}\right) \\
z^{\gamma_{1}} b_{1} \otimes z^{\gamma_{2}} b_{2} & \longmapsto z^{\gamma_{2}+H\left(b_{1} \otimes b_{2}\right)} b_{2}^{\prime} \otimes z^{\gamma_{1}-H\left(b_{1} \otimes b_{2}\right)} b_{1}^{\prime} .
\end{aligned}
$$


$H\left(b_{1} \otimes b_{2}\right)$ is called the energy function and uniquely determined up to an additive constant by the following rule:

$$
\begin{aligned}
H\left(\tilde{e}_{i}\left(b_{1} \otimes b_{2}\right)\right) & =H\left(b_{1} \otimes b_{2}\right)+1 \text { if } i=0, \varphi_{0}\left(b_{1}\right) \geq \varepsilon_{0}\left(b_{2}\right), \varphi_{0}\left(b_{2}^{\prime}\right) \geq \varepsilon_{0}\left(b_{1}^{\prime}\right), \\
& =H\left(b_{1} \otimes b_{2}\right)-1 \text { if } i=0, \varphi_{0}\left(b_{1}\right)<\varepsilon_{0}\left(b_{2}\right), \varphi_{0}\left(b_{2}^{\prime}\right)<\varepsilon_{0}\left(b_{1}^{\prime}\right), \\
& =H\left(b_{1} \otimes b_{2}\right) \quad \text { otherwise. }
\end{aligned}
$$

If we want to emphasize $B_{1} \otimes B_{2}$, we write $H_{B_{1} B_{2}}$ for $H$. Since $R^{2}=i d$, we have $H_{B_{1} B_{2}}\left(b_{1} \otimes b_{2}\right)=H_{B_{2} B_{1}}\left(b_{2}^{\prime} \otimes b_{1}^{\prime}\right)$. Note that if $B_{1}=B_{2}$, one has $b_{2}^{\prime}=b_{1}, b_{1}^{\prime}=b_{2}$.

Now let $B_{1}, B_{2}$ and $B_{3}$ be finite crystals. Then the Yang-Baxter equation holds on $\operatorname{Aff}\left(B_{1}\right) \otimes \operatorname{Aff}\left(B_{2}\right) \otimes \operatorname{Aff}\left(B_{3}\right)$.

$$
(\mathrm{id} \otimes R)(R \otimes \mathrm{id})(\mathrm{id} \otimes R)=(R \otimes \mathrm{id})(\mathrm{id} \otimes R)(R \otimes \mathrm{id}) .
$$

For $b_{i} \in B_{i}(i=1,2,3)$ let $b_{1}^{\prime}, b_{2}^{\prime}, \hat{b}_{3}, \check{b}_{3}$ be defined by

$$
\begin{array}{rlrllllll}
B_{1} \otimes B_{2} & \stackrel{\sim}{\rightarrow} B_{2} \otimes B_{1} & B_{2} \otimes B_{3} & \stackrel{\sim}{\rightarrow} & B_{3} \otimes B_{2} & B_{1} \otimes B_{3} & \stackrel{\sim}{\rightarrow} & B_{3} \otimes B_{1} \\
b_{1} \otimes b_{2} & \mapsto & b_{2}^{\prime} \otimes b_{1}^{\prime} & b_{2} \otimes b_{3} & \mapsto & \hat{b}_{3} \otimes b_{2}^{\prime \prime} & b_{1}^{\prime} \otimes b_{3} & \mapsto & \breve{b}_{3} \otimes b_{1}^{\prime \prime}
\end{array}
$$

under the isomorphism of crystals. One can check (3.1) implies

$$
H\left(b_{2} \otimes b_{3}\right)+H\left(b_{1} \otimes \hat{b}_{3}\right)=H\left(b_{1}^{\prime} \otimes b_{3}\right)+H\left(b_{2}^{\prime} \otimes \check{b}_{3}\right) .
$$

Let $\tau$ be a Dynkin diagram automorphism, i.e., a permutation on letters from $\{0,1, \cdots, n\}$. By setting $\tau\left(\sum_{i} m_{i} \Lambda_{i}\right)=\sum_{i} m_{i} \Lambda_{\tau(i)}$ one can also define an automorphism on $P^{+}$. Next let $B$ be a perfect crystal. Since $\tau$ is a Dynkin diagram automorphism, one necessarily has a unique bijection, denoted again by $\tau$, from $B$ onto itself satisfying

$$
\tilde{e}_{\tau(i)}(\tau(b))=\tau\left(\tilde{e}_{i} b\right), \quad \tilde{f}_{\tau(i)}(\tau(b))=\tau\left(\tilde{f}_{i} b\right) .
$$

Here one should understand $\tau(0)=0$. Note that (3.3) implies $\varepsilon(\tau(b))=$ $\tau(\varepsilon(b)), \varphi(\tau(b))=\tau(\varphi(b))$.

We end this subsection with

Conjecture 3.1. Let $B_{1}, B_{2}$ be perfect crystals of level $l_{1}, l_{2}\left(l_{1} \geq l_{2}\right)$. Under the isomorphism of crystals $B_{1} \otimes B_{2} \stackrel{\sim}{\rightarrow} B_{2} \otimes B_{1}$, any element $b_{1} \otimes b_{2}$ of $\left(B_{1} \otimes B_{2}\right)_{\text {min }}$ is mapped to $\sigma_{1}\left(b_{2}\right) \otimes \varphi_{B_{1}}^{-1}\left(\varphi_{B_{1}}\left(b_{1}\right)+\right.$ wt $\left.b_{2}\right)$, where $\sigma_{1}$ is the Dynkin diagram automorphism associated to the perfect crystal $B_{1}$ (see the end of Section 2.8) and $\varphi_{B_{1}}$ is the map $\varphi$ from $B_{1}$.

Remark 3.2. If each $B_{i}$ belongs to a coherent family of perfect crystals (This coherent family may depend on $i$. See also Remark 2.5.) and the map $\varepsilon \times \varphi$ : $B_{i} \rightarrow\left(P^{+}\right)^{2} ; b \mapsto(\varepsilon(b), \varphi(b))$ is injective for any $i$, then the conjecture can be shown to be true using the theory of crystals with core [KK. Such case covers all coherent families of perfect crystals listed in KKM. The conjecture is proven for any case $B_{i}=B^{k_{i}, s_{i}}$ for type $A_{n}^{(1)}$ (SS1] Theorem 7.3), although the injectivity of $\varepsilon \times \varphi$ is no longer valid.

\footnotetext{
${ }^{9}$ This $\tau$ should be distinguished from that in Section 6.1.
} 


\subsection{Set of paths}

Let $B$ be a finite crystal of level $l$. From $B$ we construct a subset of $\cdots \otimes B \otimes \cdots \otimes$ $B$ called a set of paths. First we fix a reference path $\boldsymbol{p}=\cdots \otimes \boldsymbol{b}_{j} \otimes \cdots \otimes \boldsymbol{b}_{2} \otimes \boldsymbol{b}_{1}$. For any $j, \varepsilon\left(\boldsymbol{b}_{j}\right)$ should have level $l$, and satisfy

$$
\varphi\left(\boldsymbol{b}_{j+1}\right)=\varepsilon\left(\boldsymbol{b}_{j}\right) .
$$

Set

$$
\mathcal{P}(\boldsymbol{p}, B)=\left\{p=\cdots \otimes b_{j} \otimes \cdots \otimes b_{2} \otimes b_{1} \mid b_{j} \in B, b_{J}=\boldsymbol{b}_{J} \text { for } J \gg 1\right\} .
$$

An element of $\mathcal{P}(\boldsymbol{p}, B)$ is called a path. For a path $p \in \mathcal{P}(\boldsymbol{p}, B)$ we can define the energy $E(p)$ and weight wt $p$ by

$$
\begin{aligned}
E(p) & =\sum_{j=1}^{\infty} j\left(H\left(b_{j+1} \otimes b_{j}\right)-H\left(\boldsymbol{b}_{j+1} \otimes \boldsymbol{b}_{j}\right)\right), \\
w t p & =\varphi\left(\boldsymbol{b}_{1}\right)+\sum_{j=1}^{\infty}\left(w t b_{j}-w t \boldsymbol{b}_{j}\right)-\left(E(p) / a_{0}\right) \delta,
\end{aligned}
$$

where $a_{0}$ is the 0 -th Kac label.

Suppose $B$ is perfect of level $l$. Take $\lambda \in P_{l}^{+}$and define $\boldsymbol{b}_{j}$ to be an element of $B_{\min }$ such that $\varepsilon\left(\boldsymbol{b}_{j}\right)=\sigma^{j} \lambda$. We denote the corresponding reference path by $\boldsymbol{p}^{(\lambda)}$. It is known in KMN1 that we have the following isomorphism of $P$-weighted crystals.

$$
\mathcal{P}\left(\boldsymbol{p}^{(\lambda)}, B\right) \simeq B(\lambda) .
$$

Next let $B_{i}$ be a perfect crystal of level $l_{i}$ for $i=1, \cdots, d$. One may assume $B_{i}=B^{k_{i}, t_{k_{i}} l_{i}}$ in Conjecture 2.1 with some $k_{i}\left(1 \leq k_{i} \leq n\right)$. We also assume $l_{1} \geq l_{2} \geq \cdots \geq l_{d}$. Set $B=B_{1} \otimes B_{2} \otimes \cdots \otimes B_{d}$. For $\lambda_{i} \in P_{l_{i}-l_{i+1}}^{+}(i=$ $\left.1, \cdots, d ; l_{d+1}=0\right)$ define a reference path $\boldsymbol{p}^{\left(\lambda_{1}, \cdots, \lambda_{d}\right)}=\cdots \otimes \boldsymbol{b}_{j} \otimes \cdots \otimes \boldsymbol{b}_{2} \otimes \boldsymbol{b}_{1}$ $\left(\boldsymbol{b}_{j} \in B\right)$ by

$$
\begin{gathered}
\boldsymbol{b}_{j}=\varepsilon_{B_{1}}^{-1}\left(\sigma_{1}^{j} \lambda_{1}+\sigma_{1}^{j} \sigma_{2}^{j} \lambda_{2}+\cdots+\sigma_{1}^{j} \sigma_{2}^{j} \cdots \sigma_{d}^{j} \lambda_{d}\right) \\
\otimes \varepsilon_{B_{2}}^{-1}\left(\sigma_{1}^{j-1} \sigma_{2}^{j} \lambda_{2}+\sigma_{1}^{j-1} \sigma_{2}^{j} \sigma_{3}^{j} \lambda_{3}+\cdots+\sigma_{1}^{j-1} \sigma_{2}^{j} \cdots \sigma_{d}^{j} \lambda_{d}\right) \\
\otimes \\
\quad \cdots \\
\quad \otimes \varepsilon_{B_{d}}^{-1}\left(\sigma_{1}^{j-1} \sigma_{2}^{j-1} \cdots \sigma_{d-1}^{j-1} \sigma_{d}^{j} \lambda_{d}\right) .
\end{gathered}
$$

Here $\varepsilon_{B_{i}}$ signifies the map $\varepsilon$ on $B_{i}$ and $\sigma_{i}$ is the automorphism on $P^{+}$corresponding to $B_{i}$.

Conjecture 3.3. With the notations above, we have the following isomorphism of P-weighted crystals.

$$
\mathcal{P}\left(\boldsymbol{p}^{\left(\lambda_{1}, \cdots, \lambda_{d}\right)}, B\right) \simeq B\left(\lambda_{1}\right) \otimes \cdots \otimes B\left(\lambda_{d}\right) .
$$


Remark 3.4. This conjecture is intimately related to the following. There exists an isomorphism of $P_{c l}$-weighted crystals

$$
B\left(\lambda_{1}\right) \otimes \cdots \otimes B\left(\lambda_{d}\right) \simeq\left(B\left(\sigma_{1} \lambda_{1}\right) \otimes \cdots \otimes B\left(\sigma_{1} \cdots \sigma_{d} \lambda_{d}\right)\right) \otimes B
$$

Let $u_{\lambda}$ be the highest weight element in $B(\lambda)$. Under this isomorphism, $u_{\lambda_{1}} \otimes$ $\cdots \otimes u_{\lambda_{d}}$ is mapped to $\left(u_{\sigma_{1} \lambda_{1}} \otimes \cdots \otimes u_{\sigma_{1} \cdots \sigma_{d} \lambda_{d}}\right) \otimes \boldsymbol{b}_{1}$. Thus the reference path $\boldsymbol{p}^{\left(\lambda_{1}, \cdots, \lambda_{d}\right)}$ corresponds to the highest weight element in $B\left(\lambda_{1}\right) \otimes \cdots \otimes B\left(\lambda_{d}\right)$.

Remark 3.5. One can show Remark 2.5 and Conjecture 3.1 imply this conjecture in a similar manner to the $\left(A_{n}^{(1)}, B^{1, l_{i}}\right)$ case in HKKOT]. Since Conjecture 3.1 is true for all coherent families of perfect crystals listed in [KKM] (Remark 3.2), the above conjecture is true for these cases.

This conjecture can be generalized to the case when each $B_{i}$ is of the form $B^{k_{i}, s_{i}}$ but not necessarily perfect. We prepare some notations. Set $t_{\max }=$ $\max _{1 \leq i \leq n} t_{i}$. Later in this section we consider an affine Lie algebra $\mathfrak{g}$ such that $t_{\max }>1$. To such $\mathfrak{g}$ we associate a different algebra $\mathfrak{g}^{(i)}\left(1 \leq i<t_{\max }\right)$ by

$$
\begin{aligned}
& \mathfrak{g}=B_{n}^{(1)} \Longrightarrow \mathfrak{g}^{(1)}=D_{n+1}^{(1)}, \\
& \mathfrak{g}=C_{n}^{(1)} \Longrightarrow \mathfrak{g}^{(1)}=A_{2 n-1}^{(1)}, \\
& \mathfrak{g}=F_{4}^{(1)} \Longrightarrow \mathfrak{g}^{(1)}=E_{6}^{(1)}, \\
& \mathfrak{g}=G_{2}^{(1)} \Longrightarrow \mathfrak{g}^{(1)}=B_{3}^{(1)}, \mathfrak{g}^{(2)}=D_{4}^{(1)} .
\end{aligned}
$$

Notice that there is a natural inclusion

$$
\begin{array}{ll}
\mathfrak{g} \hookrightarrow \mathfrak{g}^{(1)} & \text { for } \mathfrak{g}=B_{n}^{(1)}, C_{n}^{(1)}, F_{4}^{(1)}, \\
\mathfrak{g} \hookrightarrow \mathfrak{g}^{(1)} \hookrightarrow \mathfrak{g}^{(2)} & \text { for } \mathfrak{g}=G_{2}^{(1)} .
\end{array}
$$

Next consider an integrable $\mathfrak{g}^{(i)}$-module $L^{(i)}\left(\mu^{(i)}\right)$ with highest weight $\mu^{(i)}$. Note that $\mu^{(i)}$ is a weight with respect to the algebra $\mathfrak{g}^{(i)}$. Through the inclusion (3.5) one can decompose $L^{(i)}\left(\mu^{(i)}\right)$ into irreducible integrable $\mathfrak{g}$-modules as

$$
L^{(i)}\left(\mu^{(i)}\right)=\bigoplus_{\nu} L(\nu)^{\otimes m_{\nu}},
$$

where $\nu$ runs through the weight lattice $P$ of $\mathfrak{g}$ and $m_{\nu}$ is the multiplicity. Now define $B^{(i)}\left(\mu^{(i)}\right)$ by

$$
B^{(i)}\left(\mu^{(i)}\right)=\bigoplus_{\nu} B(\nu)^{\otimes m_{\nu}} .
$$

Recall that $B(\nu)$ is the crystal basis of $L(\nu)$.

Consider the crystal $B=B^{k_{1}, s_{1}} \otimes B^{k_{2}, s_{2}} \otimes \cdots \otimes B^{k_{d}, s_{d}}$. To the datum $\left(k_{1}, s_{1}\right), \cdots,\left(k_{d}, s_{d}\right)$, associate a set

$$
A=\left\{s_{i} / t_{k_{i}} \mid i=1, \cdots, d\right\}
$$


We decompose it into disjoint components.

$$
\begin{aligned}
A & =\bigsqcup_{j} A\left[l_{j}\right], \\
A[l] & =\left\{s_{i} / t_{k_{i}} \in A \mid\left\lceil s_{i} / t_{k_{i}}\right\rceil=l\right\} .
\end{aligned}
$$

Let $\bar{d}$ be the number of disjoint components. We arrange $l_{1}, \cdots, l_{\bar{d}}$ so that $l_{1}>\cdots>l_{\bar{d}}$ and set $l_{\bar{d}+1}=0$. For any $i(1 \leq i \leq \bar{d})$ we introduce the following crystal:

- $t_{\max }=2$,

$$
\begin{aligned}
B[i]= & B\left(\lambda_{i}\right) \otimes B^{(1)}\left(\mu_{i}^{(1)}\right) \\
& \left(\lambda_{i}: \text { level } l_{i}-l_{i+1}-1 \mathfrak{g} \text {-weight, } \mu_{i}^{(1)}: \text { level } 1 \mathfrak{g}^{(1)} \text {-weight }\right) \\
& \text { if } l_{i}-1 / 2 \in A\left[l_{i}\right] \\
= & B\left(\lambda_{i}\right) \quad\left(\lambda_{i}: \text { level } l_{i}-l_{i+1} \mathfrak{g} \text {-weight }\right) \\
& \text { otherwise. }
\end{aligned}
$$

- $t_{\max }=3$,

$$
\begin{aligned}
B[i]= & B\left(\lambda_{i}\right) \otimes B^{(2)}\left(\mu_{i}^{(2)}\right) \\
& \left(\lambda_{i}: \text { level } l_{i}-l_{i+1}-1 \mathfrak{g} \text {-weight, } \mu_{i}^{(2)}: \text { level } 1 \mathfrak{g}^{(2)} \text {-weight }\right) \\
& \text { if both } l_{i}-1 / 3 \text { and } l_{i}-2 / 3 \in A\left[l_{i}\right], \\
= & B\left(\lambda_{i}\right) \otimes B^{(1)}\left(\mu_{i}^{(1)}\right) \\
& \left(\lambda_{i}: \text { level } l_{i}-l_{i+1}-1 \mathfrak{g} \text {-weight, } \mu_{i}^{(1)}: \text { level } 1 \mathfrak{g}^{(1)} \text {-weight }\right) \\
& \text { if either } l_{i}-1 / 3 \text { or } l_{i}-2 / 3 \in A\left[l_{i}\right], \\
& B\left(\lambda_{i}\right) \quad\left(\lambda_{i}: \text { level } l_{i}-l_{i+1} \mathfrak{g} \text {-weight }\right) \\
& \text { otherwise. }
\end{aligned}
$$

Choosing such datum $(\hat{\lambda}, \hat{\mu})=\left\{\lambda_{i}, \mu_{i}^{(t)} \mid i=1, \cdots, \bar{d}, 1 \leq t<t_{\max }\right\}$, we set

$$
\mathcal{B}(\hat{\lambda}, \hat{\mu})=B[1] \otimes B[2] \otimes \cdots \otimes B[\bar{d}] .
$$

By definition, it is the crystal basis of a level $l_{1}$ integrable $U_{q}(\mathfrak{g})$-module, which is not irreducible in general. If $\lambda_{i}$ is level $0, B\left(\lambda_{i}\right)$ should be considered as the crystal basis of the trivial module and hence can be omitted.

Conjecture 3.6. For any datum $(\hat{\lambda}, \hat{\mu})$ as above, there exists a reference path $\boldsymbol{p}^{(\hat{\lambda}, \hat{\mu})}$, and we have the following isomorphism of P-weighted crystals.

$$
\mathcal{P}\left(\boldsymbol{p}^{(\hat{\lambda}, \hat{\mu})}, B\right) \simeq \mathcal{B}(\hat{\lambda}, \hat{\mu}) .
$$

We give examples of $\mathcal{B}(\hat{\lambda}, \hat{\mu})$. 
Example 3.7. $\quad$ (i) $\mathfrak{g}=C_{3}^{(1)}\left(t_{1}=t_{2}=2, t_{3}=1, t_{\max }=2, \mathfrak{g}^{(1)}=A_{5}^{(1)}\right)$

$$
\begin{aligned}
& B=B^{2,5} \otimes B^{1,5} \otimes B^{1,4} \otimes B^{2,2} \otimes B^{1,1} . \\
& A=A[3] \sqcup A[2] \sqcup A[1], \\
& A[3]=\{5 / 2\}, A[2]=\{2\}, A[1]=\{1,1 / 2\} . \\
& \mathcal{B}(\hat{\lambda}, \hat{\mu})=B^{(1)}\left(\mu_{1}^{(1)}\right) \otimes B\left(\lambda_{2}\right) \otimes B^{(1)}\left(\mu_{3}^{(1)}\right),
\end{aligned}
$$

where $\lambda_{2}$ is a level $1 C_{3}^{(1)}$-weight and $\mu_{1}^{(1)}, \mu_{3}^{(1)}$ are level $1 A_{5}^{(1)}$-weights.

(ii) $\mathfrak{g}=G_{2}^{(1)}\left(t_{1}=1, t_{2}=3, t_{\max }=3, \mathfrak{g}^{(1)}=B_{3}^{(1)}, \mathfrak{g}^{(2)}=D_{4}^{(1)}\right)$

$$
\begin{aligned}
& B=B^{2,8} \otimes B^{2,7} \otimes B^{1,1} \otimes B^{2,1} . \\
& A=A[3] \sqcup A[1], \\
& A[3]=\{8 / 3,7 / 3\}, A[1]=\{1,1 / 3\} . \\
& \mathcal{B}(\hat{\lambda}, \hat{\mu})=B\left(\lambda_{1}\right) \otimes B^{(2)}\left(\mu_{1}^{(2)}\right) \otimes B^{(1)}\left(\mu_{2}^{(1)}\right),
\end{aligned}
$$

where $\lambda_{1}$ is a level $1 G_{2}^{(1)}$-weight, $\mu_{1}^{(2)}$ level $1 D_{4}^{(1)}$-weight and $\mu_{2}^{(1)}$ level 1 $B_{3}^{(1)}$-weight.

Except for the case when $B^{k_{i}, s_{i}}$ are all perfect (3.4), we do not know how to construct the reference path $\boldsymbol{p}^{(\hat{\lambda}, \hat{\mu})}$ in general. We only give the answer for the case of $\mathfrak{g}=C_{n}^{(1)}, B=B^{1, s_{1}} \otimes \cdots \otimes B^{1, s_{d}}$ here. Let us first recall the crystal $B^{1, s}$. As a set, it reads as

$$
\begin{aligned}
B^{1, s}=\{ & \left(x_{1}, \ldots, x_{n}, \bar{x}_{n}, \ldots, \bar{x}_{1}\right) \in \mathbb{Z}^{2 n} \mid x_{i}, \bar{x}_{i} \geq 0 \\
& \left.\sum_{i=1}^{n}\left(x_{i}+\bar{x}_{i}\right) \leq s, \sum_{i=1}^{n}\left(x_{i}+\bar{x}_{i}\right) \equiv s \quad(\bmod 2)\right\} .
\end{aligned}
$$

For more details, see Example 2.5 of HKOTY1.

Now consider the crystal $B=B^{1, s_{1}} \otimes \cdots \otimes B^{1, s_{d}}\left(s_{1}>s_{2}>\cdots>s_{d}>\right.$ $\left.s_{d+1}=0\right)$. Let $l_{j}=\left\lceil s_{j} / 2\right\rceil$ and fix a datum

$$
(\hat{\lambda}, \hat{\mu})=\left(\lambda_{j}, \hat{\Lambda}_{\mu_{i}} \mid 1 \leq j \leq d, i \in\left\{j \mid s_{j} \text { is odd }\right\}\right),
$$

where $\lambda_{j}$ is a level $l_{j}-l_{j+1}$ (resp. $l_{j}-l_{j+1}-1$ ) dominant integral weight of $C_{n}^{(1)}$ if $s_{j}$ is even (resp. odd) and $\hat{\Lambda}_{\mu_{i}}$ is a fundamental weight of $A_{2 n-1}^{(1)}$. We need to define two maps $\pi, \iota . \quad \pi$ is a map from $A_{2 n-1}^{(1)}$ fundamental weights $\hat{\Lambda}_{\mu}(\mu=0, \ldots, 2 n-1)$ to $C_{n}^{(1)}$ fundamental weights given by $\pi\left(\hat{\Lambda}_{\mu}\right)=\Lambda_{\mu}$ $(0 \leq \mu \leq n),=\Lambda_{2 n-\mu}(n<\mu \leq 2 n-1)$. For a $C_{n}^{(1)}$ weight $\lambda=\sum_{i=0}^{n} \lambda^{i} \Lambda_{i}$, define $\iota(\lambda)=\left(\lambda^{1}, \cdots, \lambda^{n}, \lambda^{n}, \cdots, \lambda^{1}\right)$. Let $e_{k}$ be the $k$-th unit row-vector of dimension $2 n$. If $2 n<k<4 n, e_{k}$ should be understood as $e_{k-2 n}$. Now define an element $\boldsymbol{c}^{(j)}$ of $\left(B^{1, s_{j}}\right)^{\otimes 2 n}$ by

$$
\begin{aligned}
\boldsymbol{c}^{(j)} & =\boldsymbol{c}_{1}^{(j)} \otimes \cdots \otimes \boldsymbol{c}_{2 n}^{(j)} \quad\left(\boldsymbol{c}_{i}^{(j)} \in B^{1, s_{j}}\right), \\
\boldsymbol{c}_{i}^{(j)} & = \begin{cases}\iota\left(\sum_{j \leq k \leq d} \lambda_{k}+\sum_{j+1 \leq k \leq d, s_{k}: \text { odd }} \pi\left(\hat{\Lambda}_{\mu_{k}}\right)\right) & \text { if } s_{j} \text { is even } \\
\iota\left(\sum_{j \leq k \leq d} \lambda_{k}+\sum_{j+1 \leq k \leq d, s_{k}: \text { odd }} \pi\left(\hat{\Lambda}_{\mu_{k}}\right)\right)+e_{\mu_{j}+i} & \text { if } s_{j} \text { is odd. }\end{cases}
\end{aligned}
$$


Next applying crystal isomorphisms $B^{1, s_{i}} \otimes B^{1, s_{j}} \stackrel{\sim}{\rightarrow} B^{1, s_{j}} \otimes B^{1, s_{i}}$ as many times as needed, one defines an element $\boldsymbol{b}_{2 n} \otimes \cdots \otimes \boldsymbol{b}_{1}$ of $B^{\otimes 2 n}$ by

$$
\begin{aligned}
\left(B^{1, s_{1}}\right)^{\otimes 2 n} \otimes\left(B^{1, s_{2}}\right)^{\otimes 2 n} \otimes \cdots \otimes\left(B^{1, s_{d}}\right)^{\otimes 2 n} & \stackrel{\sim}{\rightarrow} B^{\otimes 2 n} \\
\boldsymbol{c}^{(1)} \otimes \boldsymbol{c}^{(2)} \otimes \cdots \otimes \boldsymbol{c}^{(2 n)} & \mapsto \boldsymbol{b}_{2 n} \otimes \cdots \otimes \boldsymbol{b}_{1} .
\end{aligned}
$$

Then $\boldsymbol{b}_{2 n} \otimes \cdots \otimes \boldsymbol{b}_{1}$ gives the first $2 n$ components of our reference path $\boldsymbol{p}^{(\hat{\lambda}, \hat{\nu})}$ corresponding to the datum (3.6). The other components should be determined so that they are periodic with period $2 n$. To calculate the isomorphism (3.7) explicitly, one can use an algorithm based on insertion scheme HKOT1. See also Appendix D.

We give examples of the above mentioned $\boldsymbol{b}_{2 n} \otimes \cdots \otimes \boldsymbol{b}_{1}$ for $n=2$, i.e. $\mathfrak{g}=C_{2}^{(1)}$.

Example 3.8. (i) $B=B^{1,4} \otimes B^{1,1},(\hat{\lambda}, \hat{\mu})=\left(\lambda_{1}=\Lambda_{0}, \lambda_{2}=0, \hat{\Lambda}_{\mu_{2}}=\hat{\Lambda}_{0}\right)$

$$
\begin{aligned}
& \boldsymbol{c}^{(1)}=(0,0,0,0) \otimes(0,0,0,0) \otimes(0,0,0,0) \otimes(0,0,0,0) \\
& \boldsymbol{c}^{(2)}=(1,0,0,0) \otimes(0,1,0,0) \otimes(0,0,1,0) \otimes(0,0,0,1) \\
& \boldsymbol{b}_{1}=(1,0,0,1) \otimes(0,0,0,1) \quad \boldsymbol{b}_{2}=(0,1,1,0) \otimes(0,0,1,0) \\
& \boldsymbol{b}_{3}=(1,0,0,1) \otimes(0,1,0,0) \quad \boldsymbol{b}_{4}=(0,0,0,0) \otimes(1,0,0,0)
\end{aligned}
$$

(ii) $B=B^{1,5} \otimes B^{1,3},(\hat{\lambda}, \hat{\mu})=\left(\lambda_{1}=0, \lambda_{2}=\Lambda_{2}, \hat{\Lambda}_{\mu_{1}}=\hat{\Lambda}_{3}, \hat{\Lambda}_{\mu_{2}}=\hat{\Lambda}_{1}\right)$

$$
\begin{aligned}
& \boldsymbol{c}^{(1)}=(1,1,1,2) \otimes(2,1,1,1) \otimes(1,2,1,1) \otimes(1,1,2,1) \\
& \boldsymbol{c}^{(2)}=(0,2,1,0) \otimes(0,1,2,0) \otimes(0,1,1,1) \otimes(1,1,1,0) \\
& \boldsymbol{b}_{1}=(0,1,2,0) \otimes(1,1,1,0) \quad \boldsymbol{b}_{2}=(1,2,1,1) \otimes(0,1,1,1) \\
& \boldsymbol{b}_{3}=(1,2,2,0) \otimes(0,1,2,0) \quad \boldsymbol{b}_{4}=(1,1,1,2) \otimes(0,2,1,0)
\end{aligned}
$$

\subsection{One dimensional sums}

Let $B_{i}(i=1, \cdots, d)$ be finite crystals, and consider the tensor product $B_{1} \otimes$ $\cdots \otimes B_{d}$. We define $b_{j}^{(i)}(i<j)$ by

$$
\begin{aligned}
& B_{i} \otimes \cdots \otimes B_{j-1} \otimes B_{j} \quad \stackrel{\sim}{\rightarrow} \quad B_{i} \otimes \cdots \otimes B_{j} \otimes B_{j-1} \quad \stackrel{\sim}{\rightarrow} \quad \cdots \\
& b_{i} \otimes \cdots \otimes b_{j-1} \otimes b_{j} \quad \mapsto \quad b_{i} \otimes \cdots \otimes b_{j}^{(j-1)} \otimes b_{j-1}^{\prime} \quad \mapsto \quad \cdots \\
& \cdots \quad \stackrel{\sim}{\rightarrow} B_{j} \otimes B_{i} \otimes \cdots \otimes B_{j-1} \\
& \cdots \quad \mapsto b_{j}^{(i)} \otimes b_{i}^{\prime} \otimes \cdots \otimes b_{j-1}^{\prime},
\end{aligned}
$$

and set $b_{i}^{(i)}=b_{i}$. Suppose $B_{i}=B^{k_{i}, s_{i}}(1 \leq i \leq d)$ and let $l_{i}$ be the level of the crystal $B^{k_{i}, s_{i}}$. Take $b_{i}^{\natural} \in B^{k_{i}, s_{i}}$ such that $\varphi\left(b_{i}^{\natural}\right)=l_{i} \Lambda_{0}$. Even if $B^{k_{i}, s_{i}}$ is not perfect, we expect such $b_{i}^{\natural}$ exists uniquely. It is indeed true for the $C_{n}^{(1)}$ crystal $B^{1, s}$ with odd $s$ presented in HKKOT. For later use we also define $b_{i}^{\text {ext }} \in B^{k_{i}, s_{i}}$ to be the highest weight element of the $U_{q}(\mathfrak{g})$-crystal $B\left(s_{i} \bar{\Lambda}_{k_{i}}\right)$ 
contained in $B^{k_{i}, s_{i}}$. For a finite path $p=b_{1} \otimes \cdots \otimes b_{d} \in B^{k_{1}, s_{1}} \otimes \cdots \otimes B^{k_{d}, s_{d}}$, define

$$
D(p)=\sum_{1 \leq i<j \leq d} H\left(b_{i} \otimes b_{j}^{(i+1)}\right)+\sum_{1 \leq j \leq d} H\left(b_{j}^{\natural} \otimes b_{j}^{(1)}\right) .
$$

Fix a positive integer $l$ such that $l \geq \max _{i} l_{i}$. We define a (restricted) one dimensional sum by

$$
X_{l}\left(B^{k_{1}, s_{1}} \otimes \cdots \otimes B^{k_{d}, s_{d}}, \lambda, q\right)=\sum_{p}^{*} q^{D(p)},
$$

where the sum $\sum_{p}^{*}$ is taken over all $p=b_{1} \otimes \cdots \otimes b_{d} \in B^{k_{1}, s_{1}} \otimes \cdots \otimes B^{k_{d}, s_{d}}$ satisfying $\sum_{j=1}^{d} w t b_{j}=\lambda$ and

$$
\tilde{e}_{i}^{l \delta_{i 0}+1}\left(b_{1} \otimes \cdots \otimes b_{d}\right)=0 \quad \forall i .
$$

(3.10) can be rewritten as local conditions.

$$
\tilde{e}_{i}^{\left\langle h_{i}, l \Lambda_{0}+w t b_{1}+\cdots+w t b_{j-1}\right\rangle+1} b_{j}=0 \quad \forall i, 1 \leq j \leq d .
$$

We formally consider the $l=\infty$ case, where the condition $(\overline{3.10})$ for $i=0$ is vacant. $X_{\infty}(B, \lambda, q)$ was called classically restricted in [HKOTY1]. It is easy to see from the definition that

$$
X_{\infty}\left(B^{k_{1}, s_{1}} \otimes \cdots \otimes B^{k_{d}, s_{d}}, \lambda, 1\right)=\left[W_{s_{1}}^{\left(k_{1}\right)} \otimes \cdots \otimes W_{s_{d}}^{\left(k_{d}\right)}: \lambda\right] .
$$

Here $W_{s}^{(k)}$ is the corresponding finite-dimensional $U_{q}^{\prime}(\mathfrak{g})$-module of $B^{k, s}$ and

$$
[M: \mu]=\operatorname{dim} \mathbb{C}\left\langle v \in M \mid w t v=\mu, e_{i} v=0 \forall i \neq 0\right\rangle,
$$

where $e_{i}$ denotes the root vector corresponding to the simple root $\alpha_{i}$.

Proposition 3.9. $X_{l}\left(B^{k_{1}, s_{1}} \otimes \cdots \otimes B^{k_{d}, s_{d}}, \lambda, q\right)$ does not depend on the order of the crystals $B^{k_{1}, s_{1}}, \cdots, B^{k_{d}, s_{d}}$ in the tensor product.

Proof. We set $B_{i}=B^{k_{i}, s_{i}}(i=1, \cdots, d)$. It is enough to check that $X_{l}$ remains unchanged under the switch of $B_{i}$ and $B_{i+1}$ in the tensor product. Suppose $b_{i} \otimes b_{i+1}$ is mapped to $b_{i+1}^{\prime} \otimes b_{i}^{\prime}$ under the isomorphism $B_{i} \otimes B_{i+1} \simeq B_{i+1} \otimes B_{i}$. For $p=b_{1} \otimes \cdots \otimes b_{d} \in B_{1} \otimes \cdots \otimes B_{d}$ define $p^{\prime}=b_{1} \otimes \cdots \otimes b_{i+1}^{\prime} \otimes b_{i}^{\prime} \otimes \cdots \otimes b_{d} \in$ $B_{1} \otimes \cdots \otimes B_{i+1} \otimes B_{i} \otimes \cdots \otimes B_{d}$. Note that wt $p=w t p^{\prime}$ and $p$ satisfies (3.10) if and only if so does $p^{\prime}$. Hence it is enough to show $D(p)=D\left(p^{\prime}\right)$. Set

$$
D_{j}(p)=\sum_{k=1}^{j-1} H\left(b_{k} \otimes b_{j}^{(k+1)}\right), \quad \hat{D}(p)=\sum_{j=1}^{d} H\left(b_{j}^{\natural} \otimes b_{j}^{(1)}\right) .
$$

Then $D(p)=\sum_{j=1}^{d} D_{j}(p)+\hat{D}(p)$. Since $\hat{D}(p)=\hat{D}\left(p^{\prime}\right)$ is evident, we are left to show the following: 
(i) $D_{j}(p)=D_{j}\left(p^{\prime}\right) \quad(j \neq i, i+1)$,

(ii) $D_{i}(p)+D_{i+1}(p)=D_{i}\left(p^{\prime}\right)+D_{i+1}\left(p^{\prime}\right)$.

(i) for $j<i$ is trivial. To check (i) for $j>i+1$ use (3.1), in particular, (3.2) with $b_{1} \rightarrow b_{i}, b_{2} \rightarrow b_{i+1}, b_{3} \rightarrow b_{j}^{(i+2)}$. For (ii) notice that $D_{i+1}(p)=$ $D_{i}\left(p^{\prime}\right)+H\left(b_{i} \otimes b_{i+1}\right), D_{i+1}\left(p^{\prime}\right)=D_{i}(p)+H\left(b_{i+1}^{\prime} \otimes b_{i}^{\prime}\right)$.

Conjecture 3.10. We have

(1) $q^{-D^{e x t}} X_{\infty}\left(B^{k_{1}, s_{1}} \otimes \cdots \otimes B^{k_{d}, s_{d}}, \lambda, q\right)=M_{\infty}\left(W_{s_{1}}^{\left(k_{1}\right)} \otimes \cdots \otimes W_{s_{d}}^{\left(k_{d}\right)}, \lambda, q\right)$

$$
\text { for } \lambda \in \bar{P}^{+} \text {, }
$$

(2) $q^{-D^{e x t}} X_{l}\left(B^{k_{1}, s_{1}} \otimes \cdots \otimes B^{k_{d}, s_{d}}, 0, q\right)=M_{l}\left(W_{s_{1}}^{\left(k_{1}\right)} \otimes \cdots \otimes W_{s_{d}}^{\left(k_{d}\right)}, 0, q\right)$, where $D^{e x t}=D\left(b_{1}^{e x t} \otimes \cdots \otimes b_{d}^{e x t}\right)$ and $M_{l}$ is defined in (4.5).

At present a proof is available only for $A_{n}^{(1)}$ case [KSS, SS1].

\subsection{Representation theoretical meaning}

We explain a representation theoretical meaning of our one dimensional sum $X_{l}(B, \lambda, q)$. Set $B=B_{1} \otimes \cdots \otimes B_{d}$. We first assume all $B_{i}$ 's are perfect. Let $l_{i}$ be the level of $B_{i}$. Assume $l_{1} \geq l_{2} \geq \cdots \geq l_{d} \geq l_{d+1}=0$. Write $b^{*}=b_{1} \otimes \cdots \otimes b_{d}$ for an element of $B$ and fix $\boldsymbol{b}_{2}$ as in (3.4) with $\lambda_{i}=\sigma_{1}^{-1} \cdots \sigma_{i}^{-1}\left(l_{i}-l_{i+1}\right) \Lambda_{0}$.

Proposition 3.11. If Conjecture 3.1 is true, then $H_{B B}\left(\boldsymbol{b}_{2} \otimes b^{*}\right)-D\left(b^{*}\right)$ is independent of $b^{*}$.

We need three lemmas.

Lemma 3.12 (HKKOT] Proposition 3.1). Let $B_{1}, B_{2}$ be finite crystals. Set $B=B_{1} \otimes B_{2}$, then

$$
\begin{aligned}
H_{B B}\left(\left(b_{1} \otimes b_{2}\right) \otimes\left(b_{1}^{\prime} \otimes b_{2}^{\prime}\right)\right)= & H_{B_{1} B_{2}}\left(b_{1} \otimes b_{2}\right)+H_{B_{1} B_{1}}\left(\tilde{b}_{1} \otimes b_{1}^{\prime}\right) \\
& +H_{B_{2} B_{2}}\left(b_{2} \otimes \tilde{b}_{2}^{\prime}\right)+H_{B_{1} B_{2}}\left(b_{1}^{\prime} \otimes b_{2}^{\prime}\right) .
\end{aligned}
$$

Here $\tilde{b}_{1}, \tilde{b}_{2}^{\prime}$ are defined as

$$
\begin{aligned}
B_{1} \otimes B_{2} & \stackrel{\sim}{\rightarrow} B_{2} \otimes B_{1} \\
b_{1} \otimes b_{2} & \mapsto \tilde{b}_{2} \otimes \tilde{b}_{1} \\
b_{1}^{\prime} \otimes b_{2}^{\prime} & \mapsto \tilde{b}_{2}^{\prime} \otimes \tilde{b}_{1}^{\prime} .
\end{aligned}
$$

Lemma 3.13. Consider the tensor product of finite crystals $B=B_{1} \otimes B_{2} \otimes$ $\cdots \otimes B_{d}$. Set $B_{>1}=B_{2} \otimes \cdots \otimes B_{d}$. For an element $b_{1} \otimes \cdots \otimes b_{d} \in B$, define $b_{1}^{\langle j\rangle}(1 \leq j \leq d) b y$

$$
\begin{array}{cll}
B_{1} \otimes B_{2} \otimes \cdots \otimes B_{j} & \stackrel{\sim}{\rightarrow} B_{2} \otimes \cdots \otimes B_{j} \otimes B_{1} \\
b_{1} \otimes b_{2} \otimes \cdots \otimes b_{j} & \mapsto & \tilde{b}_{2} \otimes \cdots \otimes \tilde{b}_{j} \otimes b_{1}^{\langle j\rangle} .
\end{array}
$$


We understand $b_{1}^{\langle 1\rangle}=b_{1}$. Then we have

$$
H_{B_{1} B>1}\left(b_{1} \otimes\left(b_{2} \otimes \cdots \otimes b_{d}\right)\right)=\sum_{2 \leq j \leq d} H_{B_{1} B_{j}}\left(b_{1}^{\langle j-1\rangle} \otimes b_{j}\right) .
$$

Lemma 3.14. We retain the notations in the previous lemma. Consider isomorphisms of crystals

$$
\begin{aligned}
B_{1} \otimes B_{2} \otimes \cdots \otimes B_{j-1} \otimes B_{j} & \stackrel{\sim}{\rightarrow} B_{2} \otimes \cdots \otimes B_{j-1} \otimes B_{j} \otimes B_{1} \\
b_{1} \otimes b_{2} \otimes \cdots \otimes b_{j-1} \otimes b_{j} & \mapsto \tilde{b}_{2} \otimes \cdots \otimes \tilde{b}_{j-1} \otimes \tilde{b}_{j} \otimes b_{1}^{\langle j\rangle} \\
& \stackrel{\sim}{\rightarrow} B_{2} \otimes \cdots \otimes B_{i-1} \otimes B_{j} \otimes B_{i} \otimes \cdots \otimes B_{j-1} \otimes B_{1} \\
& \mapsto \tilde{b}_{2} \otimes \cdots \otimes \tilde{b}_{i-1} \otimes \tilde{b}_{j}^{(i)} \otimes \cdots \\
& \stackrel{\sim}{\rightarrow} B_{j} \otimes B_{2} \otimes \cdots \otimes B_{j-1} \otimes B_{1} \\
& \mapsto \tilde{b}_{j}^{(2)} \otimes \cdots
\end{aligned}
$$

Then we have $\tilde{b}_{j}^{(2)}=b_{j}^{(1)}$ and $\sum_{i=2}^{j-1} H_{B_{i} B_{j}}\left(\tilde{b}_{i} \otimes \tilde{b}_{j}^{(i+1)}\right)+H_{B_{1} B_{j}}\left(b_{1}^{\langle j-1\rangle} \otimes b_{j}\right)=$ $\sum_{i=1}^{j-1} H_{B_{i} B_{j}}\left(b_{i} \otimes b_{j}^{(i+1)}\right)$.

The last two lemmas can be shown by using the Yang-Baxter equation (3.1).

Proof of Proposition 3.1 . The notations in the lemmas are retained. Write $\boldsymbol{b}_{2}=\bar{b}_{1} \otimes \cdots \otimes \bar{b}_{d}, \bar{b}_{i} \in B_{i}$. Let $\bar{b}_{i} \otimes \bar{b}_{i+1} \otimes \cdots \otimes \bar{b}_{d}$ be mapped to $\bar{b}_{i+1}^{\prime} \otimes \cdots \otimes \bar{b}_{d}^{\prime} \otimes \bar{b}_{i}^{\prime}$ under the isomorphism $B_{i} \otimes B_{i+1} \otimes \cdots \otimes B_{d} \stackrel{\sim}{\rightarrow} B_{i+1} \otimes \cdots \otimes B_{d} \otimes B_{i}$. Then Conjecture 3.1 implies $\bar{b}_{i}^{\prime}=b_{i}^{\natural}$.

We prove by induction on $d$. Using Lemma 3.12 and the induction hypothesis, one has

$$
\begin{aligned}
H_{B B}\left(\boldsymbol{b}_{2} \otimes b^{*}\right)= & H_{B_{1} B_{1}}\left(b_{1}^{\natural} \otimes b_{1}\right)+\sum_{2 \leq i<j \leq d} H_{B_{i} B_{j}}\left(\tilde{b}_{i} \otimes \tilde{b}_{j}^{(i+1)}\right) \\
& +\sum_{2 \leq j \leq d} H_{B_{j} B_{j}}\left(b_{j}^{\natural} \otimes \tilde{b}_{j}^{(2)}\right)+H_{B_{1} B_{>1}}\left(b_{1} \otimes\left(b_{2} \otimes \cdots \otimes b_{d}\right)\right) .
\end{aligned}
$$

Here in the equality we omitted $b^{*}$-independent terms. Lemma 3.13 and 3.14 now complete the proof.

Proposition 3.11 implies that under the embedding

$$
\begin{aligned}
B & \hookrightarrow \mathcal{P}\left(\boldsymbol{p}^{\left(\lambda_{1}, \cdots, \lambda_{d}\right)}, B\right) \simeq B\left(\lambda_{1}\right) \otimes \cdots \otimes B\left(\lambda_{d}\right) \\
b^{*} & \mapsto \cdots \otimes \boldsymbol{b}_{j} \otimes \cdots \otimes \boldsymbol{b}_{2} \otimes b^{*},
\end{aligned}
$$

the weight is given by $l_{1} \Lambda_{0}+w t b^{*}-\left(D\left(b^{*}\right)-D\left(\boldsymbol{b}_{1}\right)\right) \delta$. Here $\lambda_{i}=\sigma_{1}^{-1} \cdots \sigma_{i}^{-1}\left(l_{i}-\right.$ $\left.l_{i+1}\right) \Lambda_{0}$ and $\boldsymbol{b}_{j}$ is the $j$-th component of the reference path $\boldsymbol{p}^{\left(\lambda_{1}, \cdots, \lambda_{d}\right)}$. Let $\gamma$ be the minimal integer such that $\sigma_{i}^{\gamma}=i d$ for all $i=1, \cdots, d$. For a positive integer $L$ such that $L \equiv 0(\bmod \gamma)$, we consider a similar embedding

$$
\begin{array}{cl}
B^{\otimes L} & \hookrightarrow \mathcal{P}\left(\boldsymbol{p}^{\left(\lambda_{1}^{0}, \cdots, \lambda_{d}^{0}\right)}, B\right) \simeq B\left(\lambda_{1}^{0}\right) \otimes \cdots \otimes B\left(\lambda_{d}^{0}\right) \\
b^{L} \otimes \cdots \otimes b^{1} & \mapsto \cdots \otimes \boldsymbol{b}_{j} \otimes \cdots \otimes \boldsymbol{b}_{L+1} \otimes b^{L} \otimes \cdots \otimes b^{1} .
\end{array}
$$


This time $\lambda_{i}^{0}=\left(l_{i}-l_{i+1}\right) \Lambda_{0}$ and $\boldsymbol{b}_{j}$ is redefined as the $j$-th component of the reference path $\boldsymbol{p}^{\left(\lambda_{1}^{0}, \cdots, \lambda_{d}^{0}\right)}$. Under the embedding the weight is given by $l_{1} \Lambda_{0}+\sum_{j=1}^{L} w t b^{j}-\left(D\left(b^{L} \otimes \cdots \otimes b^{1}\right)-D\left(\boldsymbol{b}_{L} \otimes \cdots \otimes \boldsymbol{b}_{1}\right)\right) \delta$. In view of (3.10), one has

$$
\begin{aligned}
\lim _{\substack{L \rightarrow \infty \\
L \equiv 0(\bmod \gamma)}} q^{-D\left(\boldsymbol{b}_{L} \otimes \cdots \otimes \boldsymbol{b}_{1}\right)} X_{\infty}\left(B^{\otimes L}, \lambda, q\right) \\
\quad=\sum_{j}\left[\bigotimes_{i=1}^{d} L\left(\left(l_{i}-l_{i+1}\right) \Lambda_{0}\right): l_{1} \Lambda_{0}+\lambda-j \delta\right] q^{j a_{0}}
\end{aligned}
$$

Note that in $\left[\bigotimes_{i=1}^{d} L\left(\lambda_{i}^{0}\right): \mu\right], \mu$ is a weight in $P$ as opposed to $\lambda$ in (3.11) in $\bar{P}$. Let $l$ be an integer such that $l \geq l_{1}$. We also have

$$
\begin{gathered}
\lim _{\substack{L \rightarrow \infty \\
L \equiv 0 \bmod \gamma)}} q^{-D\left(\boldsymbol{b}_{L} \otimes \cdots \otimes \boldsymbol{b}_{1}\right)} X_{l}\left(B^{\otimes L}, \lambda, q\right) \\
=\sum_{j}\left[\left[L\left(\left(l-l_{1}\right) \Lambda_{0}\right) \otimes \bigotimes_{i=1}^{d} L\left(\left(l_{i}-l_{i+1}\right) \Lambda_{0}\right): l \Lambda_{0}+\lambda-j \delta\right]\right] q^{j a_{0}} .
\end{gathered}
$$

Here $[[M, \mu]]=\operatorname{dim} \mathbb{C}\left\langle v \in M \mid w t v=\mu, e_{i} v=0 \forall i\right\rangle$.

Generalization of such interpretation is straightforward to the case when $B_{i}$ is not necessarily perfect. In view of Conjecture 3.6, let $\mathcal{L}(\hat{\lambda}, \hat{\mu})$ be the corresponding integrable $U_{q}(\mathfrak{g})$-module of $\mathcal{B}(\hat{\lambda}, \hat{\mu})$. One only needs to replace $\bigotimes_{i=1}^{d} L\left(\left(l_{i}-l_{i+1}\right) \Lambda_{0}\right)$ with $\mathcal{L}(\hat{\lambda}, \hat{\mu})$.

\section{$4 \quad$ Fermionic formula}

\subsection{Conventions on $\mathfrak{g}_{\overline{0}}$}

From Section 2.1 we remind that $\left\{\bar{\Lambda}_{a}\right\}_{a=1}^{n}$ and $\left\{\alpha_{a}\right\}_{a=1}^{n}$ are the sets of fundamental weights and simple roots of $\stackrel{\circ}{\mathfrak{g}}$, and $\bar{P}=\mathbb{Z} \bar{\Lambda}_{1} \oplus \cdots \oplus \mathbb{Z} \bar{\Lambda}_{n}$ denotes its weight lattice. $\left\{\alpha_{a}\right\}$ has been normalized as $\left(\alpha_{a} \mid \alpha_{a}\right)=2 r$ if $\alpha_{a}$ is a long root. See Exercise 6.1 of [Kad. In Sections 1 , 5 and 6 we find it convenient to further introduce the simple roots $\left\{\tilde{\alpha}_{a}\right\}_{a=1}^{n}$ and the fundamental weights $\left\{\tilde{\Lambda}_{a}\right\}_{a=1}^{n}$ of $\mathfrak{g}_{0}$, and let $\tilde{P}=\mathbb{Z} \tilde{\Lambda}_{1} \oplus \cdots \oplus \mathbb{Z} \tilde{\Lambda}_{n}$. We equip the bilinear form $(\cdot \mid \cdot)^{\prime}$ on $\tilde{P}$ which is again normalized as $\left(\tilde{\alpha}_{a} \mid \tilde{\alpha}_{a}\right)^{\prime}=2 r$ if $\tilde{\alpha}_{a}$ is a long root of $\mathfrak{g}_{0}$. (For $A_{2}^{(2)}$, we understand that the unique simple root $\tilde{\alpha}_{1}$ of $\mathfrak{g}_{0}=B_{1}$ is short. See below.) Actually the situation $\stackrel{\circ}{\mathfrak{g}} \neq \mathfrak{g}_{0}$ happens only for $\mathfrak{g}=A_{2 n}^{(2)}$, where $\stackrel{\circ}{\mathfrak{g}}=C_{n}$ and $\mathfrak{g}_{0}=B_{n}$. Comparing the normalizations in the above and Section 2.1, we find that $\alpha_{a}, \bar{\Lambda}_{a}$ and $(\cdot \mid \cdot)$ may be identified with $\tilde{\alpha}_{a}, \tilde{\Lambda}_{a}$ and $(\cdot \mid \cdot)^{\prime}$ if $\mathfrak{g} \neq A_{2 n}^{(2)}$. In order to deal with the $A_{2 n}^{(2)}$ case uniformly, we introduce a $\mathbb{Z}$-linear map $\iota: \bar{P} \rightarrow \tilde{P}$ by

$$
\iota\left(\bar{\Lambda}_{a}\right)=\epsilon_{a} \tilde{\Lambda}_{a} \quad 1 \leq a \leq n,
$$


where $\epsilon_{a}$ is specified as

$$
\epsilon_{a}=\left\{\begin{array}{ll}
2 & \text { if } \mathfrak{g}=A_{2 n}^{(2)} \\
1 & \text { otherwise }
\end{array} \text { and } a=n .\right.
$$

Note that (4.1) induces $\iota\left(\alpha_{a}\right)=\epsilon_{a} \tilde{\alpha}_{a}$. One can check $\left(\iota\left(\alpha_{b}\right) \mid \iota\left(\alpha_{b}\right)\right)^{\prime}=a_{0}\left(\alpha_{b} \mid \alpha_{b}\right)$ for any $1 \leq b \leq n$. For example in $A_{2 n}^{(2)}$ case, the both sides are equal to 8 if $b=n$ and 4 otherwise. Especially for $A_{2}^{(2)}(n=1)$, we have $\left(\tilde{\alpha}_{1} \mid \tilde{\alpha}_{1}\right)^{\prime}=2$ and $\left(\alpha_{1} \mid \alpha_{1}\right)=4$. Now that the definitions being clear, in the rest of the paper (mostly in the rest of this section and Sections 5 and 6) we shall simply write $(\cdot \mid \cdot)^{\prime}$ also as $(\cdot \mid \cdot)$. This will not cause a confusion because the entries shall be limited to the self-explanatory objects $\alpha_{a}, \bar{\Lambda}_{a}, \tilde{\alpha}_{a}, \tilde{\Lambda}_{a}, \iota\left(\bar{\Lambda}_{a}\right)$, etc. We will also make abbreviations $|\mu|^{2}=(\mu \mid \mu)$ for $\mu$ belonging to either $\stackrel{\circ}{\mathfrak{g}}$ or $\mathfrak{g}_{\overline{0}}$ root systems. With these conventions one finds

$$
\tilde{\alpha}_{a}=\sum_{b=1}^{n} \frac{\epsilon_{b} t_{b}}{t_{b}^{\vee}}\left(\tilde{\alpha}_{a} \mid \tilde{\alpha}_{b}\right) \tilde{\Lambda}_{b}, \quad \alpha_{a}=\sum_{b=1}^{n} \frac{a_{0} t_{b}}{\epsilon_{b} t_{b}^{\vee}}\left(\alpha_{a} \mid \alpha_{b}\right) \bar{\Lambda}_{b}
$$

which tells that the matrices $\left(\frac{\epsilon_{a} t_{a}}{t_{a}^{r}}\left(\tilde{\alpha}_{a} \mid \tilde{\alpha}_{b}\right)\right)_{1 \leq a, b \leq n}$ and $\left(\frac{a_{0} t_{a}}{\epsilon_{a} t_{a}^{r}}\left(\alpha_{a} \mid \alpha_{b}\right)\right)_{1 \leq a, b \leq n}$ coincide with the Cartan matrices of $\mathfrak{g}_{\overline{0}}$ and $\stackrel{\circ}{\mathfrak{g}}$, respectively for any $\mathfrak{g}$. In particular,

$$
\frac{2 t_{b}^{\vee}}{t_{b}\left|\tilde{\alpha}_{b}\right|^{2}}=\frac{a_{0} t_{b}\left|\alpha_{b}\right|^{2}}{2 t_{b}^{\vee}}=\epsilon_{b} \quad 1 \leq b \leq n
$$

is valid.

\section{$4.2 \quad M_{l}(W, \lambda, q)$ and $\tilde{M}_{l}(W, \lambda, q)$}

For $m \in \mathbb{Z}_{\geq 0}$ and $p \in \mathbb{Z}$, we define

$$
\left[\begin{array}{c}
p+m \\
m
\end{array}\right]_{q}=\frac{\left(q^{p+1} ; q\right)_{\infty}\left(q^{m+1} ; q\right)_{\infty}}{(q ; q)_{\infty}\left(q^{p+m+1} ; q\right)_{\infty}}
$$

where $(x ; q)_{\infty}=\prod_{j=0}^{\infty}\left(1-x q^{j}\right)$. The quantity 4.4 is the $q$-binomial coefficient for $p \in \mathbb{Z}_{\geq 0}$, vanishing for $-m \leq p \leq-1$, and is equal to $\left(-q^{p+(m+1) / 2}\right)^{m}\left[\begin{array}{c}-p-1 \\ m\end{array}\right]_{q}$ for $p \leq-m-1$. In the $q \rightarrow 1$ limit it becomes

$$
\left[\begin{array}{c}
p+m \\
m
\end{array}\right]_{1}=\frac{\Gamma(p+m+1)}{\Gamma(p+1) \Gamma(m+1)} .
$$

We shall also use the notation $(q)_{k}=(q ; q)_{\infty} /\left(q^{k+1} ; q\right)_{\infty}$ for $k \in \mathbb{Z}_{\geq 0}$.

For any $l \in \mathbb{Z}_{\geq 1}$, we introduce the index set

$$
\begin{aligned}
& H_{l}=\left\{(a, i) \mid 1 \leq a \leq n, 1 \leq i \leq t_{a} l\right\} \\
& \bar{H}_{l}=\left\{(a, i) \mid 1 \leq a \leq n, 1 \leq i \leq t_{a} l-1\right\} .
\end{aligned}
$$


We will also use $H_{l}$ formally with $l=\infty$.

Given $\left\{\nu_{j}^{(a)} \in \mathbb{Z}_{\geq 0} \mid(a, j) \in H_{l}\right\}$, we set $W=\bigotimes_{(a, j) \in H_{l}}\left(W_{j}^{(a)}\right)^{\otimes \nu_{j}^{(a)}}$. When $l=\infty$ we assume that $\sum_{j \geq 1} \nu_{j}^{(a)}<\infty$ for all $1 \leq a \leq n$.

Let $\lambda=\lambda_{1} \bar{\Lambda}_{1}+\cdots+\lambda_{n} \bar{\Lambda}_{n}$ be an element of $\bar{P}$. We define the fermionic form $M_{l}(W, \lambda, q)$ for $\mathfrak{g}$ by

$$
\begin{aligned}
M_{l}(W, \lambda, q)= & \sum_{\{m\}} q^{c(\{m\})} \prod_{(a, i) \in H_{l}}\left[\begin{array}{c}
p_{i}^{(a)}+m_{i}^{(a)} \\
m_{i}^{(a)}
\end{array}\right]_{q_{a}}, \\
c(\{m\})= & \frac{1}{2} \sum_{(a, j),(b, k) \in H_{l}}\left(\tilde{\alpha}_{a} \mid \tilde{\alpha}_{b}\right) \min \left(t_{b} j, t_{a} k\right) m_{j}^{(a)} m_{k}^{(b)} \\
& \quad-\sum_{a=1}^{n} t_{a}^{\vee} \sum_{1 \leq j, k \leq t_{a} l} \min (j, k) \nu_{j}^{(a)} m_{k}^{(a)}, \\
p_{i}^{(a)}= & \sum_{j=1}^{t_{a} l} \nu_{j}^{(a)} \min (i, j)-\frac{1}{t_{a}^{\vee}} \sum_{(b, k) \in H_{l}}\left(\tilde{\alpha}_{a} \mid \tilde{\alpha}_{b}\right) \min \left(t_{b} i, t_{a} k\right) m_{k}^{(b)},
\end{aligned}
$$

where $q_{a}$ is given by

$$
q_{a}=q^{t_{a}^{\vee}}
$$

The sum $\sum_{\{m\}}$ is taken over $\left\{m_{i}^{(a)} \in \mathbb{Z}_{\geq 0} \mid(a, i) \in H_{l}\right\}$ satisfying

$$
\begin{gathered}
p_{j}^{(a)} \geq 0 \text { for all }(a, j) \in H_{l}, \\
\sum_{(a, i) \in H_{l}} i m_{i}^{(a)} \tilde{\alpha}_{a}=\iota\left(\sum_{(a, i) \in H_{l}} i \nu_{i}^{(a)} \bar{\Lambda}_{a}-\lambda\right) .
\end{gathered}
$$

From (4.7) it is easy to see that $(4.10)$ is equivalent to $p_{t_{a} l}^{(a)}=\lambda_{a}$ for any $\mathfrak{g}$. In view of this and the constraint (4.9) one has

$$
M_{l}(W, \lambda, q)=0 \quad \text { unless } \lambda \in\left(\sum_{(a, i) \in H_{l}} i \nu_{i}^{(a)} \bar{\Lambda}_{a}-\sum_{a=1}^{n} \mathbb{Z}_{\geq 0} \iota^{-1}\left(\tilde{\alpha}_{a}\right)\right) \cap \bar{P}^{+} .
$$

It is easy to see the properties

$$
p_{i}^{(a)}=-\frac{1}{t_{a}^{\vee}} \frac{\partial c(\{m\})}{\partial m_{i}^{(a)}}, \quad M_{l}(W, \lambda, q) \in q^{-\epsilon} \mathbb{Z}_{\geq 0}\left[q^{-a_{0}}\right]
$$

where $\epsilon \equiv \sum_{a=1}^{n} a\left(\sum_{i=1}^{l} i \nu_{i}^{(a)}-\lambda_{a}\right) \bmod a_{0} \mathbb{Z}$.

Except (5.4) in the next section, we will actually consider $M_{l}(W, \lambda, q)$ only for $\lambda=0$ when $l<\infty$. Using $p_{t_{a} l}^{(a)}=0$ and eliminating $m_{t_{a} l}^{(a)}$ by

$$
\sum_{(a, i) \in H_{l}} i m_{i}^{(a)} \tilde{\alpha}_{a}=\sum_{(a, i) \in H_{l}} i \nu_{i}^{(a)} \iota\left(\bar{\Lambda}_{a}\right)
$$


one can rewrite the fermionic form for $l<\infty$ as

$$
\begin{aligned}
M_{l}\left(W, 0, q^{-1}\right)= & \sum_{\{m\}} q^{\bar{c}(\{m\})} \prod_{(a, i) \in \bar{H}_{l}}\left[\begin{array}{c}
p_{i}^{(a)}+m_{i}^{(a)} \\
m_{i}^{(a)}
\end{array}\right]_{q_{a}}, \\
\bar{c}(\{m\})= & \frac{1}{2} \sum_{(a, j),(b, k) \in \bar{H}_{l}}\left(\tilde{\alpha}_{a} \mid \tilde{\alpha}_{b}\right) K_{t_{b} j, t_{a} k}^{\left(t_{a} t_{b} l\right)} m_{j}^{(a)} m_{k}^{(b)} \\
& \quad+\frac{1}{2 l}\left|\sum_{(a, i) \in H_{l}} i \nu_{i}^{(a)} \iota\left(\bar{\Lambda}_{a}\right)\right|^{2} \\
p_{i}^{(a)}= & \sum_{j=1}^{t_{a} l-1} K_{i, j}^{\left(t_{a} l\right)} \nu_{j}^{(a)}-\frac{1}{t_{a}^{\vee}} \sum_{(b, k) \in \bar{H}_{l}}\left(\tilde{\alpha}_{a} \mid \tilde{\alpha}_{b}\right) K_{t_{b} i, t_{a} k}^{\left(t_{a} t_{b} l\right)} m_{k}^{(b)},
\end{aligned}
$$

where the quantity $K_{j k}^{(l)}$ is defined by

$$
K_{j k}^{(l)}=\min (j, k)-\frac{j k}{l} .
$$

The sum $\sum_{\{m\}}$ is now taken over $\left\{m_{i}^{(a)} \in \mathbb{Z}_{\geq 0} \mid(a, i) \in \bar{H}_{l}\right\}$ satisfying $p_{i}^{(a)} \geq 0$ for all $(a, i) \in \bar{H}_{l}$ and the condition that $m_{t_{a} l}^{(a)}$ determined from (4.12) is a non-negative integer. When $l=\infty$, a similar calculation leads to

$$
\begin{aligned}
& M_{\infty}\left(\bigotimes_{a, j} W_{j}^{(a) \otimes \nu_{j}^{(a)}}, \sum_{a, j} j \nu_{j}^{(a)} \bar{\Lambda}_{a}-\mu, q^{-1}\right) \\
& =\sum_{\{m\}} q^{\hat{c}(\{m\})} \prod_{1 \leq a \leq n, i \geq 1}\left[\begin{array}{c}
p_{i}^{(a)}+m_{i}^{(a)} \\
m_{i}^{(a)}
\end{array}\right]_{q_{a}}, \\
& \hat{c}(\{m\})=\frac{1}{2} \sum_{1 \leq a, b \leq n} \sum_{j, k \geq 1}\left(\tilde{\alpha}_{a} \mid \tilde{\alpha}_{b}\right) \min \left(t_{b} j, t_{a} k\right) m_{j}^{(a)} m_{k}^{(b)}, \\
& p_{i}^{(a)}=\sum_{j \geq 1} \nu_{j}^{(a)} \min (i, j)-\frac{1}{t_{a}^{\vee}} \sum_{b=1}^{n} \sum_{k \geq 1}\left(\tilde{\alpha}_{a} \mid \tilde{\alpha}_{b}\right) \min \left(t_{b} i, t_{a} k\right) m_{k}^{(b)} .
\end{aligned}
$$

Here the sum $\sum_{\{m\}}$ is taken over $\left\{m_{i}^{(a)} \in \mathbb{Z}_{\geq 0} \mid 1 \leq a \leq n, i \geq 1\right\}$ satisfying $p_{i}^{(a)} \geq 0$ for all $1 \leq a \leq n, i \geq 1$ and $\sum_{a=1}^{n} \sum_{j \geq 1} j m_{j}^{(a)} \tilde{\alpha}_{a}=\iota(\mu)$.

Let us present the examples from $\mathfrak{g}$ of rank 1 .

Example 4.1. Take $\mathfrak{g}=A_{1}^{(1)}$ and $W=\bigotimes_{j} W_{j}^{\otimes \nu_{j}}$. (We understand $W_{j}=$ $W_{j}^{(1)}$.) Then we have

$$
\begin{aligned}
M_{\infty}\left(W, \lambda_{1} \bar{\Lambda}_{1}, q^{-1}\right) & =\sum q^{\sum_{i, j \geq 1} \min (i, j) m_{i} m_{j}} \prod_{i \geq 1}\left[\begin{array}{c}
p_{i}+m_{i} \\
m_{i}
\end{array}\right]_{q}, \\
p_{i} & =\sum_{j \geq 1}\left(\nu_{j}-2 m_{j}\right) \min (i, j) .
\end{aligned}
$$


Here $\lambda_{1}$ is a non-negative integer congruent to $\sum_{j} j \nu_{j} \bmod 2$. The sum is taken over $m_{1}, m_{2}, \ldots \in \mathbb{Z}_{\geq 0}$ such that $\forall p_{j} \geq 0$ and $\sum_{j \geq 1} j m_{j}=\left(\sum_{j} j \nu_{j}-\lambda_{1}\right) / 2$. For $W=W_{1}^{\otimes 2} \otimes W_{2}$, the non-zero ones are given by

$$
M_{\infty}\left(W, 4 \bar{\Lambda}_{1}, q^{-1}\right)=1, \quad M_{\infty}\left(W, 2 \bar{\Lambda}_{1}, q^{-1}\right)=q+q^{2}, \quad M_{\infty}\left(W, 0, q^{-1}\right)=q^{2} .
$$

Example 4.2. Take $\mathfrak{g}=A_{2}^{(2)}$ and $W=\bigotimes_{j} W_{j}^{\otimes \nu_{j}}$. (We understand $W_{j}=$ $W_{j}^{(1)}$.) Then we have

$$
\begin{aligned}
M_{\infty}\left(W, \lambda_{1} \bar{\Lambda}_{1}, q^{-1}\right) & =\sum q^{\sum_{i, j \geq 1} \min (i, j) m_{i} m_{j}} \prod_{i \geq 1}\left[\begin{array}{c}
p_{i}+m_{i} \\
m_{i}
\end{array}\right]_{q^{2}}, \\
p_{i} & =\sum_{j \geq 1}\left(\nu_{j}-m_{j}\right) \min (i, j) .
\end{aligned}
$$

Here $\lambda_{1}$ is a non-negative integer. The sum is taken over $m_{1}, m_{2}, \ldots \in \mathbb{Z}_{>0}$ such that $\forall p_{j} \geq 0$ and $\sum_{j \geq 1} j m_{j}=\sum_{j} j \nu_{j}-\lambda_{1}$. For $W=W_{1}^{\otimes 2} \otimes W_{2}$, the non-zero ones are give by

$M_{\infty}\left(W, 4 \bar{\Lambda}_{1}, q^{-1}\right)=1$,

$$
M_{\infty}\left(W, 3 \bar{\Lambda}_{1}, q^{-1}\right)=q+q^{3}+q^{5}, \quad M_{\infty}\left(W, 2 \bar{\Lambda}_{1}, q^{-1}\right)=q^{2}+2 q^{4}+2 q^{6}+q^{8},
$$$$
M_{\infty}\left(W, \bar{\Lambda}_{1}, q^{-1}\right)=q^{3}+2 q^{5}+2 q^{7}+2 q^{9}, \quad M_{\infty}\left(W, 0, q^{-1}\right)=q^{4}+q^{6}+2 q^{8}+q^{10} .
$$

It is natural to consider a relative of $M_{l}$ defined by 10

$$
\tilde{M}_{l}(W, \lambda, q)=(4.5)-(4.10 \text { without the constraint }(4.9) \text {. }
$$

\subsection{Symmetry and recursion relation}

Compared with $M_{l}$ in (4.11), $\tilde{M}$ introduced in (4.20) has a nice symmetry as emphasized in HKOTY1] when $r=1$. In fact our computer experiments indicate ${ }^{11}$

Conjecture 4.3.

$$
\begin{aligned}
M_{l}(W, \lambda, q) & =\tilde{M}_{l}(W, \lambda, q), \quad \text { for } l \leq \infty \text { and } \lambda \in \bar{P}^{+}, \\
\tilde{M}_{\infty}(W, w(\lambda+\bar{\rho})-\bar{\rho}, q) & =\operatorname{det} w \tilde{M}_{\infty}(W, \lambda, q) \quad \text { for any } \lambda \in \bar{P},
\end{aligned}
$$

where $\bar{\rho}=\bar{\Lambda}_{1}+\cdots+\bar{\Lambda}_{n}$ and $w$ is any element of the Weyl group of $\stackrel{\circ}{\mathfrak{g}}$.

\footnotetext{
${ }^{10} \tilde{M}_{l}$ here was denoted by $N_{l}$ in eq. (4.16) of [HKOTY1.

11 The former equality in Conjecture 4.3 reduces to eq.(4.22) in HKOTY1 when $\lambda=0$. We withdraw the claim in the last sentence on p.260 of [HKOTY1].
} 
If $\mathfrak{g}$ is non-exceptional or $D_{4}^{(3)}$, i.e., $\mathfrak{g}=A_{n}^{(1)}, B_{n}^{(1)}, C_{n}^{(1)}, D_{n}^{(1)}, A_{2 n}^{(2)}, A_{2 n-1}^{(2)}, D_{n+1}^{(2)}$ or $D_{4}^{(3)}$, the last property can indeed be proved at $q=1$. It is based on the argument in Remark 8.8 of [HKOTY1] together with the existence of the Weyl group invariant solution to the $Q$-system established in Theorem 7.1 in HKOTY1 and Theorem 6.2 in this paper.

Consider the nontwisted simply laced algebras $\mathfrak{g}=X_{N}^{(1)}=A_{n}^{(1)}, D_{n}^{(1)}(N=$ $n)$ and $E_{6}^{(1)}$. Let $\sigma$ be the order $r$ automorphism of the $X_{N}$ Dynkin diagram as in Section 2.1. We have $r=3$ if $\mathfrak{g}=D_{4}^{(1)}$ and $r=2$ otherwise. We shall let $\sigma$ act on the index set $\{1, \ldots, N\}$ as well as on the weight lattice of $\stackrel{\circ}{\mathfrak{g}}=X_{N}$. From the definition of the fermionic formula, it is straightforward to see

$$
M_{l}\left(\bigotimes_{(a, j) \in H_{l}}\left(W_{j}^{(a)}\right)^{\otimes \nu_{j}^{(a)}}, \lambda, q\right)=M_{l}\left(\bigotimes_{(a, j) \in H_{l}}\left(W_{j}^{(\sigma(a))}\right)^{\otimes \nu_{j}^{(a)}}, \sigma(\lambda), q\right),
$$

which also implies (B.1).

Next we present a recursion relation of the fermionic form $M_{l}(W, \lambda, q)$ with respect to $W$. For $a, b \in\{1, \ldots, n\}$ and $j, k \in \mathbb{Z}$, we set (cf. eq.(A.6) in [KN])

$$
\begin{aligned}
& B_{b k, a j}=2 \min \left(t_{a} k, t_{b} j\right)-\min \left(t_{a} k, t_{b}(j+1)\right)-\min \left(t_{a} k, t_{b}(j-1)\right) \\
& =\left\{\begin{array}{cl}
2 \delta_{k, 2 j}+\delta_{k, 2 j+1}+\delta_{k, 2 j-1} & \left(t_{b}, t_{a}\right)=(2,1) \\
3 \delta_{k, 3 j}+2 \delta_{k, 3 j+1}+2 \delta_{k, 3 j-1} & \left(t_{b}, t_{a}\right)=(3,1) \\
+\delta_{k, 3 j+2}+\delta_{k, 3 j-2} & \\
t_{b} \delta_{t_{a} k, t_{b} j} & \text { otherwise. }
\end{array}\right.
\end{aligned}
$$

Proposition 4.4. Fix $(a, j) \in \bar{H}_{l}$ arbitrarily. Given any $\lambda \in \bar{P}^{+}$and $W=$ $\bigotimes_{(b, k) \in H_{l}}\left(W_{k}^{(b)}\right)^{\otimes \nu_{k}^{(b)}}$, set

$$
\begin{aligned}
& W_{1}=W_{j}^{(a)} \otimes W_{j}^{(a)} \otimes W, \\
& W_{2}=W_{j+1}^{(a)} \otimes W_{j-1}^{(a)} \otimes W, \\
& W_{3}=\bigotimes_{(b, k) \in H_{l}} W_{k}^{(b) \otimes\left(2 \delta_{a b} \delta_{j k}-\left(\tilde{\alpha}_{a} \mid \tilde{\alpha}_{b}\right) B_{a j, b k} / t_{b}^{\vee}\right)} \otimes W .
\end{aligned}
$$

Then we have

$$
\begin{aligned}
M_{l}\left(W_{1}, \lambda, q\right) & =M_{l}\left(W_{2}, \lambda, q\right)+q_{a}^{-\theta} M_{l}\left(W_{3}, \lambda, q\right), \\
\theta & =\left(2-\epsilon_{a}^{-1}\right) j+\sum_{k=1}^{t_{a} l} \nu_{k}^{(a)} \min (j, k) .
\end{aligned}
$$

The same recursion relation holds also for $\tilde{M}_{l}(W, \lambda, q)$.

When $j=1$ we understand that $W_{2}=W_{2}^{(a)} \otimes W$. The proof is similar to that for Theorem 6.1 in HKOTY1] for $r=1$. In view of (6.14), $W_{1}, W_{2}$ and $W_{3}$ with trivial $W$ correspond to the three terms in the $Q$-system (6.1). 


\subsection{Limit of fermionic formulae}

Recall that our fermionic formulae are connected to the $1 \mathrm{dsums}$ by Conjecture 3.10, where the latters are indeed affine Lie algebra characters in the infinite lattice limit as in (3.12) and (3.13). Motivated by this fact, we here investigate the behavior of our fermionic formulae, either of argument $q$ or $q^{-1}$, when the quantum space data $\left\{\nu_{j}^{(a)}\right\}$ gets large. It turns out that they tend to an intriguing form ${ }^{2}$. Roughly, they break into a sum of products in which each factor is again identified with a fermionic form associated with "smaller" levels or algebras. It will be interesting to seek physical interpretations of such phenomena. See [ANOT, BLS2, NY2] and reference therein.

Proposition 4.5 (Spinon character formula). Assume that $l / t_{b} \in \mathbb{Z}$. For any $1 \leq b \leq n$ we have

$$
\begin{aligned}
\lim q^{-c_{0}} M_{\infty}\left(W_{l}^{(b) \otimes L}, \lambda, q\right) & =\sum_{\zeta} \frac{M_{\infty}\left(W(\zeta), \lambda, q^{-1}\right) M_{l / t_{b}}\left(W(\zeta), 0, q^{-1}\right)}{\left(q_{1}\right)_{\zeta_{1}} \cdots\left(q_{n}\right)_{\zeta_{n}}} \\
W(\zeta) & =\bigotimes_{a=1}^{n} W_{1}^{(a) \otimes \zeta_{a}}
\end{aligned}
$$

Here $\lim$ means the limit $L \rightarrow \infty$ so that $L\left(\iota\left(\bar{\Lambda}_{a}\right) \mid \iota\left(\bar{\Lambda}_{b}\right)\right) /\left(t_{a}^{\vee} t_{b}\right) \in \mathbb{Z}$ for $1 \leq$ $\forall a \leq n . c_{0}=-L^{2} l\left|\iota\left(\bar{\Lambda}_{b}\right)\right|^{2} /\left(2 t_{b}\right) \in \mathbb{Z}$. The sum is taken over $\zeta_{1}, \ldots, \zeta_{n} \in \mathbb{Z}_{\geq 0}$ such that $\sum_{a=1}^{n} \zeta_{a} l\left(\bar{\Lambda}_{a}\right) \in \oplus_{a=1}^{n} \mathbb{Z}_{\geq 0} \tilde{\alpha}_{a}$.

Under Conjecture 2.1 (1), the condition $l / t_{b} \in \mathbb{Z}$ is equivalent to assuming that the crystal $B^{b, l}$ is perfect. Thus Proposition 4.5 covers all the twisted cases and Theorem 5.4 in [HKOTY1], to which the proof is similar. The quantity $c_{0}$ is the minimum of the quadratic form $c(\{m\})$ in (4.6) at $m_{i}^{(a)}=$ $\delta_{i, t_{a} l / t_{b}} L\left(\iota\left(\bar{\Lambda}_{a}\right) \mid \iota\left(\bar{\Lambda}_{b}\right)\right) /\left(t_{a}^{\vee} t_{b}\right)$.

For any $\mathfrak{g}$, one can extend Proposition 4.5 to the situation where $W_{l}^{(b)}$ is replaced by a tensor product $W_{s_{1}}^{\left(k_{1}\right)} \otimes \cdots \otimes W_{s_{d}}^{\left(k_{d}\right)}$. To present it, we introduce a generalization of (4.13) depending on a subset $S \subset \bar{H}_{l}$.

$$
\begin{aligned}
& M_{l}^{S}\left(\bigotimes_{(a, j) \in H_{l}}\left(W_{j}^{(a)}\right)^{\otimes \nu_{j}^{(a)}}, q^{-1}\right) \\
& =\sum_{\{m\}} q^{\bar{c}(\{m\})} \prod_{(a, i) \in \bar{H}_{l} \backslash S}\left[\begin{array}{c}
p_{i}^{(a)}+m_{i}^{(a)} \\
m_{i}^{(a)}
\end{array}\right] \prod_{q_{a}} \frac{1}{(a, i) \in S},
\end{aligned}
$$

where $\bar{c}(\{m\})$ and $p_{i}^{(a)}$ are given by the same formulae as (4.14) and 4.15$)$. The sum $\sum_{\{m\}}$ is now taken over $\left\{m_{i}^{(a)} \in \mathbb{Z}_{\geq 0} \mid(a, i) \in \bar{H}_{l} \backslash S\right\}$ and $\left\{m_{i}^{(a)} \in \mathbb{Z} \mid\right.$ $(a, i) \in S\}$ satisfying $p_{i}^{(a)} \geq 0$ for all $(a, i) \in \bar{H}_{l}$ and the condition that $m_{t_{a} l}^{(a)}$

\footnotetext{
12 Convergence in the sense of the $q$-adic topology.
} 
determined from (4.12) is a non-negative integer. 4.24) reduces to (4.13) when $S=\emptyset$.

Given the quantum space $\left(W_{s_{1}}^{\left(k_{1}\right)} \otimes \cdots \otimes W_{s_{d}}^{\left(k_{d}\right)}\right)^{\otimes L}$ and the associated fermionic form (4.13), let $\left\{m_{c r}\right\}=\left\{m_{i, c r}^{(a)}(\in \mathbb{R})\right\}$ be the critical point of the corresponding quadratic form (4.14). We exclusively consider those $L$ such that $\forall m_{i, c r}^{(a)} \in \mathbb{Z}$. With the help of Remark 5.3[3 in HKOTY1 (see also eq.(3.5b-d) in [Ku]), we find for any $\mathfrak{g}$ that

$$
\begin{aligned}
& \left\{(a, i) \mid m_{i, c r}^{(a)}>0\right\}=S_{s_{1}}^{\left(k_{1}\right)} \cup \cdots \cup S_{s_{d}}^{\left(k_{d}\right)}, \\
& S_{s}^{(k)}=\left\{\begin{array}{l}
\left\{\left(a, \frac{t_{a} s}{t_{k}}\right) \mid 1 \leq a \leq n\right\} \quad \text { for } \frac{s}{t_{k}} \in \mathbb{Z}, \\
\left\{\left(a, \frac{t_{a}\left(s-s_{0}\right)}{t_{k}}\right),\left(a, \frac{t_{a}\left(s-s_{0}+t_{k}\right)}{t_{k}}\right) \mid 1 \leq a \leq n\right\} \\
\cup\left\{(a, s) \mid 1 \leq a \leq n, t_{a}=t_{k}(>1)\right\} \quad \text { for } \frac{s}{t_{k}} \notin \mathbb{Z},
\end{array}\right.
\end{aligned}
$$

where $s_{0} \equiv s \bmod t_{k} \mathbb{Z}$ and $1 \leq s_{0} \leq t_{k}-1$. It is easy to check that $\{(a, i) \mid$ $\left.m_{i, c r}^{(a)}>0\right\} \subset H_{l}$ for $l$ defined by $l=\max \left\{\left\lceil s_{i} / t_{k_{i}}\right\rceil \mid 1 \leq i \leq d\right\}$.

Proposition 4.6. With $L$ and $l$ as above, we have the following for any $\mathfrak{g}$.

$$
\begin{aligned}
& \lim _{L \rightarrow \infty} q^{-\bar{c}\left(\left\{m_{c r}\right\}\right)} M_{\infty}\left(\left(W_{s_{1}}^{\left(k_{1}\right)} \otimes \cdots \otimes W_{s_{d}}^{\left(k_{d}\right)}\right)^{\otimes L}, \lambda, q\right) \\
& =\sum_{\zeta} \frac{M_{\infty}\left(W(\zeta), \lambda, q^{-1}\right) M_{l}^{S}\left(W(\zeta), q^{-1}\right)}{\left(q_{1}\right)_{\zeta_{1}} \cdots\left(q_{n}\right)_{\zeta_{n}}}, \\
& S=\left\{(a, i) \mid m_{i, c r}^{(a)}>0\right\} \backslash\left\{\left(a, t_{a} l\right) \mid 1 \leq a \leq n\right\},
\end{aligned}
$$

where the sum $\sum_{\zeta}$ is over $\zeta_{1}, \ldots, \zeta_{n} \in \mathbb{Z}_{\geq 0}$ such that $\sum_{a=1}^{n} \zeta_{a} \iota\left(\bar{\Lambda}_{a}\right) \in \bigoplus_{a=1}^{n} \mathbb{Z}_{\geq 0} \tilde{\alpha}_{a}$, and $W(\zeta)$ is defined by (4.23).

Earlier Proposition 4.5 corresponds to the case $S=\emptyset$. (Not $M_{l / t_{b}}^{S}$ here but $M_{l}^{S}$ as above.)

Remark 4.7. When $\lambda=0$, Proposition 4.6 admits a level truncation. Let $l^{\prime}(\geq l)$ be an integer. Under the same setting as Proposition 4.6, we have

$$
\begin{aligned}
& \lim _{L \rightarrow \infty} q^{-\bar{c}\left(\left\{m_{c r}\right\}\right)} M_{l^{\prime}}\left(\left(W_{s_{1}}^{\left(k_{1}\right)} \otimes \cdots \otimes W_{s_{d}}^{\left(k_{d}\right)}\right)^{\otimes L}, 0, q\right) \\
& =\sum_{\zeta} \frac{M_{l^{\prime}-l}\left(W(\zeta), 0, q^{-1}\right) M_{l}^{S}\left(W(\zeta), q^{-1}\right)}{\left(q_{1}\right)_{\zeta_{1}} \cdots\left(q_{n}\right)_{\zeta_{n}}} .
\end{aligned}
$$

In terms of appropriate summation variables 14 , Remark 4.7 reproduces the many expressions argued in KKMM2, BLS2, NY2, HKKOTY for simply-laced $\mathfrak{g}$. In the next two examples we let $\left(C_{i j}\right)_{i, j=1}^{l-1}$ denote the Cartan matrix of $A_{l-1}$.

13 The first line on the RHS therein should be corrected as $\left\{\left(a, \frac{t_{a} s}{t_{r}}\right) \mid 1 \leq a \leq n\right\}$ for $\frac{s}{t_{r}} \in \mathbb{Z}$.

${ }^{14}$ Typically, one switches from the summation over $\left\{m_{i}^{(a)}\right\}$ to $\left\{p_{i}^{(a)}\right\}$. 
Example 4.8. Take $\mathfrak{g}=A_{1}^{(1)}$ and $1 \leq s \leq l-1$. Then we have

$$
\begin{aligned}
& \lim _{\substack{L \rightarrow \infty \\
L \in 2 \mathbb{Z}}} q^{L^{2} s / 4} M_{\infty}\left(W_{s}^{(1) \otimes L}, 0, q\right) \\
& =\sum_{\{p\}} q^{\frac{1}{4} \sum_{i, j \in \bar{H}_{l}} C_{i j} p_{i} p_{j}} \frac{1}{(q)_{p_{s}}} \prod_{\substack{1 \leq i \leq l-1 \\
i \neq s}}\left[\begin{array}{c}
p_{i}-\frac{1}{2} \sum_{j=1}^{l-1} C_{i j} p_{j} \\
p_{i}
\end{array}\right]_{q},
\end{aligned}
$$

where the sum $\sum_{\{p\}}$ extends over $p_{1}, \ldots, p_{l-1} \in 2 \mathbb{Z}_{\geq 0}$ such that $\sum_{j=1}^{l-1} C_{i j} p_{j} \leq 0$ for all $1 \leq i \leq l-1$ except $i=s$.

The RHS in the above agrees with the section 4.1 of [KKMM2] for example.

Example 4.9. Take $\mathfrak{g}=A_{2}^{(2)}$ and $1 \leq s \leq l-1$. Then we have

$$
\begin{aligned}
& \lim _{L \rightarrow \infty} q^{L^{2} s} M_{\infty}\left(W_{s}^{(1) \otimes L}, 0, q\right) \\
& =\sum_{\{p\}} q^{\sum_{i, j \in \bar{H}_{l}} C_{i j} p_{i} p_{j}} \frac{1}{\left(q^{2}\right) p_{s}} \prod_{\substack{1 \leq i \leq l-1 \\
i \neq s}}\left[\begin{array}{c}
p_{i}-\sum_{j=1}^{l-1} C_{i j} p_{j} \\
p_{i}
\end{array}\right]_{q^{2}},
\end{aligned}
$$

where the sum $\sum_{\{p\}}$ extends over $p_{1}, \ldots, p_{l-1} \in \mathbb{Z}_{\geq 0}$ such that $\sum_{j=1}^{l-1} C_{i j} p_{j} \leq 0$ for all $1 \leq i \leq l-1$ except $i=s$.

The spinon character formulae in Propositions 4.5, 4.6 and Remark 4.7 describe the limit of fermionic forms with argument $q$. Now we turn to the other limit where $q$ is replaced by $q^{-1}$. We first prepare some notations. To save the space we set

$$
\mathcal{M}_{q, \nu}^{(a)}(\mathfrak{g} \mid \mu)=M_{\infty}\left(\bigotimes_{j \geq 1} W_{j}^{(a) \otimes \nu_{j}}, \sum_{j \geq 1} j \nu_{j} \bar{\Lambda}_{a}-\mu, q\right)
$$

Here we have exhibited the dependence on the affine Lie algebra $\mathfrak{g}$ explicitly. The index $1 \leq a \leq n$ specifies a vertex of the Dynkin diagram of $\stackrel{\circ}{\mathfrak{g}}, \nu=\left\{\nu_{k}\right\}_{k \geq 1}$ is a sequence of non-negative integers and $\mu$ is an element of $\oplus_{c=1}^{n} \mathbb{Z}_{\geq 0} \alpha_{c} / \epsilon_{c}$. In the sequel we let $D(X)$ denote the Dynkin diagram of $X$. Let $\mathfrak{g}$ be an affine Lie algebra of rank $n$ and $\left\{t_{i}^{\vee} \mid i \in I\right\}$ be the associated data (2.1). Fix an index $1 \leq b \leq n$, and remove the two vertices 0 and $b$ and all the edges connected to them from the Dynkin diagram $D(\mathfrak{g})$. The result is a disjoint union of connected diagrams $D_{a}$ labeled by the indices $1 \leq a \leq n$ satisfying $\left(\alpha_{a} \mid \alpha_{b}\right)<0$. To each of them we assign an affine Lie subalgebra $\mathfrak{g}^{*} \subset \mathfrak{g}$ uniquely determined by the following condition:

$$
\begin{gathered}
\mathfrak{g}^{*}=A_{\epsilon_{a} n^{\prime}}^{\left(\epsilon_{a}\right)} \quad \text { if } D_{a}=D\left(A_{n^{\prime}}\right) \text { for some } n^{\prime} \\
\left(D\left(\mathfrak{g}^{*}\right),\left\{t_{c}^{\vee}(c \neq 0) \text { of } \mathfrak{g}^{*}\right\}\right)=\left(D_{a},\left\{t_{c}^{\vee} \text { of } \mathfrak{g} \mid c \text { belonging to } D_{a}\right\}\right) \quad \text { otherwise. }
\end{gathered}
$$


See Table 2 for the definition of the classical subalgebra $\stackrel{\circ}{\mathfrak{g}}^{*} \subset \mathfrak{g}^{*}$. The symbol $\epsilon_{a}$ in (4.2) actually becomes 2 only when $\mathfrak{g}=A_{2 n}^{(2)},(a, b)=(n, n-1)$ hence $n^{\prime}=1$ in the above. When regarding $D\left(\stackrel{\mathfrak{g}}{*}^{*}\right)$ as a subdiagram of $D(\mathfrak{g})$, the labeling of vertices within the former along Table 2 can become different from the restriction of the latter. So we let $a^{*}(\neq 0)$ denote the label within $\mathfrak{g}^{*}$ corresponding to the $a$ for $\mathfrak{g}$. Let further $\mu^{*}$ be the projection of $\mu$ to the lattice $\oplus_{c=1}^{n} \mathbb{Z}_{\geq 0} \alpha_{c} / \epsilon_{c}$ for $\stackrel{\circ}{\mathfrak{g}}^{*}$ according to $\stackrel{\circ}{\mathfrak{g}}^{*} \subset \stackrel{\circ}{\mathfrak{g}}^{\text {冏. }}$.

Proposition 4.10. Fix $1 \leq b \leq n, l \in \mathbb{Z}_{\geq 1}$ and $\mu=\mu_{1} \alpha_{1}+\cdots+\mu_{n} \alpha_{n}$. With the above definitions of $\mathfrak{g}, a^{*}$ and $\mu^{*}$, we have

$$
\begin{aligned}
& \lim _{L \rightarrow \infty} M_{\infty}\left(W_{l}^{(b) \otimes L}, L l \bar{\Lambda}_{b}-\mu, q^{-1}\right) \\
& =\sum_{\nu} \frac{q^{t_{b}^{\vee} \psi(\nu) / \epsilon_{b}}}{\prod_{i \geq 1}\left(q_{b}\right)_{\nu_{i}}} \prod_{\substack{1 \leq a \leq n \\
\left(\alpha_{a} \mid \alpha_{b}\right)<0}} \mathcal{M}_{q^{*}, \nu^{*}}^{\left(a^{*}\right)}\left(\mathfrak{g}^{*} \mid \mu^{*}\right), \\
& \psi(\nu)=\sum_{i, j \geq 1} \min (i, j) \nu_{i} \nu_{j}, \\
& \nu^{*}=\left\{\nu_{j}^{*}\right\}_{j \geq 1}, \quad \nu_{j}^{*}=-\frac{\left(\tilde{\alpha}_{a} \mid \tilde{\alpha}_{b}\right)}{t_{a}^{\vee}} \sum_{k \geq 1} \nu_{k} B_{b k, a j} \quad\left(\in \mathbb{Z}_{\geq 0}\right), \\
& q^{*}=\left\{\begin{array}{l}
q_{a} \quad \text { if } \mathfrak{g}^{*}=A_{n^{\prime}}^{(1)} \text { for some } n^{\prime} \\
q \quad \text { otherwise }
\end{array}\right.
\end{aligned}
$$

where the sum in (4.27) is taken over $\nu_{1}, \nu_{2}, \ldots \in \mathbb{Z}_{\geq 0}$ such that

$$
\sum_{i \geq 1} i \nu_{i}=\epsilon_{b} \mu_{b}
$$

Explicit forms of (4.27) are available in Appendix B. Note that the results are totally independent of $l$.

Proof. We start from the expression (4.17). In the limit $L \rightarrow \infty$, one has $p_{i}^{(b)} \rightarrow$ $\infty$ for all $i$. Thus by setting $\nu_{i}=m_{i}^{(b)}$, the factor $\prod_{i}\left[\begin{array}{c}p_{i}^{(b)}+m_{i}^{(b)} \\ m_{i}^{(b)}\end{array}\right]_{q_{b}}$ gives rise to $1 / \prod_{i}\left(q_{b}\right)_{\nu_{i}}$. The summand $\frac{1}{2}\left(\tilde{\alpha}_{b} \mid \tilde{\alpha}_{b}\right) \sum_{j, k} \min \left(t_{b} j, t_{b} k\right) m_{j}^{(b)} m_{k}^{(b)}$ contained in the quadratic form (4.18) thereby becomes $t_{b}^{\vee} \psi(\nu) / \epsilon_{b}$ on account of (4.3). For an $a$ such that $\left(\alpha_{a} \mid \alpha_{b}\right)<0$ (hence $a \neq b$ and $\left(\tilde{\alpha}_{a} \mid \tilde{\alpha}_{b}\right)<0$ ), write $p_{i}^{(a)}$ in (4.19) as

$$
p_{i}^{(a)}=-\frac{\left(\tilde{\alpha}_{a} \mid \tilde{\alpha}_{b}\right)}{t_{a}^{\vee}} \sum_{k \geq 1} \min \left(t_{b} i, t_{a} k\right) \nu_{k}-\frac{1}{t_{a}^{\vee}} \sum_{c \neq b} \sum_{k \geq 1}\left(\tilde{\alpha}_{a} \mid \tilde{\alpha}_{c}\right) \min \left(t_{c} i, t_{a} k\right) m_{k}^{(c)},
$$

15 Depending on the labelings in the two algebras, one can have $\left(\alpha_{i} \text { for } \stackrel{\circ}{\mathfrak{g}}\right)^{*}=\alpha_{i^{\prime}}$ for $\stackrel{\circ}{\mathfrak{g}^{*}}$ with $i \neq i^{\prime}$ in general. Concrete formulae are available in Appendix B for each $\mathfrak{g}$. 
where the $c$-sum actually extends over only the vertices of $D_{a}$. To read off the quantum space data $\nu^{*}$ for $\mathfrak{g}^{*}$ from this, one identifies the first terms here with that for $p_{i}^{\left(a^{*}\right)}$ from (4.19), leading to

$$
-\frac{\left(\tilde{\alpha}_{a} \mid \tilde{\alpha}_{b}\right)}{t_{a}^{\vee}} \sum_{k \geq 1} \min \left(t_{a} k, t_{b} i\right) \nu_{k}=\sum_{j \geq 1} \min (i, j) \nu_{j}^{*} .
$$

Solving this one finds (4.29). By a direct case check, the other $q$-binomial factors and the remaining terms in the quadratic form (4.18) can be matched with the contributions from the product of $\mathcal{M}_{q^{*}, \nu^{*}}^{\left(a^{*}\right)}$ 's.

\section{$5 \quad q$-series $N_{l}(\lambda, q)$}

We keep the convention $\tilde{\alpha}_{a} \in \tilde{P}$ and (.|.) explained in Section 4.1.

For $l \in \mathbb{Z}_{\geq 1}$ and $\lambda \in \bar{P}$, we introduce a $q$-series $N_{l}(\lambda, q)$ by

$$
\begin{aligned}
& N_{l}(\lambda, q)=\frac{1}{\prod_{a=1}^{n}\left(q_{a}\right)_{\infty}} \sum_{\{m\}} \frac{q^{\phi(\{m\})}}{\prod_{(a, j) \in \bar{H}_{l}}\left(q_{a}\right)_{m_{j}^{(a)}}}, \\
& \phi(\{m\})=\frac{1}{2} \sum_{(a, j),(b, k) \in \bar{H}_{l}}\left(\tilde{\alpha}_{a} \mid \tilde{\alpha}_{b}\right) K_{t_{b} j, t_{a} k}^{\left(t_{a} t_{b} l\right)} m_{j}^{(a)} m_{k}^{(b)},
\end{aligned}
$$

where $q_{a}$ and $K_{j, k}^{(l)}$ are defined in 4.8 and 4.16 ), respectively. The sum $\sum_{\{m\}}$ is taken over $\left\{m_{j}^{(a)} \in \mathbb{Z}_{\geq 0} \mid(a, j) \in \bar{H}_{l}\right\}$ satisfying

$$
\begin{aligned}
& \sum_{(a, j) \in \bar{H}_{l}} j m_{j}^{(a)} \tilde{\alpha}_{a} \equiv \iota(\lambda) \quad \bmod l M, \\
& M=\oplus_{a=1}^{n} \mathbb{Z} t_{a} \tilde{\alpha}_{a} .
\end{aligned}
$$

The sublattice $M \subset \tilde{P}$ is essentially the one introduced in the section 6.5 of Kac 16 . From the definition it follows that

$$
\begin{array}{ll}
N_{l}(\lambda, q)=N_{l}(\lambda+l \beta, q) & \text { for } \beta \in M, \\
N_{l}(\lambda, q) \in q^{\epsilon^{\prime}} \mathbb{Z}_{\geq 0}\left[\left[q^{a_{0}}\right]\right], & \epsilon^{\prime} \equiv-\frac{|\iota(\lambda)|^{2}}{2 l}+\sum_{a=1}^{n} a \lambda_{a} \quad \bmod a_{0} \mathbb{Z} .
\end{array}
$$

The $q$-series $N_{l}(\lambda, q)$ is related to the fermionic formula in the previous section via

$$
N_{l}(\lambda, q)=\lim q^{-\frac{|\iota(\lambda)|^{2}}{2 l}} M_{l}\left(W, \sum_{(a, j) \in H_{l}} j \nu_{j}^{(a)} \bar{\Lambda}_{a}-\lambda, q^{-1}\right)
$$

\footnotetext{
${ }^{16}$ This $M$ should not be confused with a fermionic form.
} 
where $M_{l}$ can also be replaced with $\tilde{M}_{l}$. Here the quantum space data is given by $W=\bigotimes_{(a, j) \in H_{l}}\left(W_{j}^{(a)}\right)^{\otimes \nu_{j}^{(a)}}$, and the limit is taken with respect to $\left\{\nu_{j}^{(a)}\right\}$ so that $\sum_{j \geq 1} \nu_{j}^{(a)} \rightarrow \infty$ for all $1 \leq a \leq n$.

Let us present the examples from $\mathfrak{g}$ of rank 1 .

Example 5.1. Take $\mathfrak{g}=A_{1}^{(1)}$, for which the formula reads

$$
N_{l}\left(\lambda_{1} \bar{\Lambda}_{1}, q\right)=\frac{1}{(q)_{\infty}} \sum_{\{m\}} \frac{q^{\sum_{1 \leq i, j \leq l-1} K_{i, j}^{(l)} m_{i} m_{j}}}{\prod_{j=1}^{l-1}(q)_{m_{j}}},
$$

where $\lambda_{1} \in 2 \mathbb{Z}$ and the sum is over $m_{1}, \ldots, m_{l-1} \in \mathbb{Z}_{\geq 0}$ such that $\sum_{j=1}^{l-1} j m_{j} \equiv$ $\lambda_{1} / 2 \bmod l \mathbb{Z}$. See (4.16) for $K_{i, j}^{(l)}$. We have

$$
\begin{aligned}
& N_{2}\left(\lambda_{1} \bar{\Lambda}_{1}, q\right) \\
& =1+q+3 q^{2}+5 q^{3}+10 q^{4}+16 q^{5}+28 q^{6}+43 q^{7}+70 q^{8}+\cdots \quad \text { if } \lambda \in 4 \mathbb{Z}, \\
& =q^{\frac{1}{2}}\left(1+2 q+4 q^{2}+7 q^{3}+13 q^{4}+21 q^{5}+35 q^{6}+55 q^{7}+86 q^{8}+\cdots\right) \quad \text { if } \lambda_{1} \notin 4 \mathbb{Z} . \\
& N_{3}\left(\lambda_{1} \bar{\Lambda}_{1}, q\right) \\
& =1+q+3 q^{2}+6 q^{3}+12 q^{4}+21 q^{5}+39 q^{6}+64 q^{7}+108 q^{8}+\cdots \quad \text { if } \lambda_{1} \in 6 \mathbb{Z}, \\
& =q^{\frac{2}{3}}\left(1+2 q+5 q^{2}+9 q^{3}+18 q^{4}+31 q^{5}+55 q^{6}+90 q^{7}+149 q^{8}+\cdots\right) \quad \text { if } \lambda_{1} \notin 6 \mathbb{Z} .
\end{aligned}
$$

Example 5.2. Take $\mathfrak{g}=A_{2}^{(2)}$, for which the formula reads

$$
N_{l}\left(\lambda_{1} \bar{\Lambda}_{1}, q\right)=\frac{1}{\left(q^{2}\right)_{\infty}} \sum_{\{m\}} \frac{q^{\sum_{1 \leq i, j \leq l-1} K_{i, j}^{(l)} m_{i} m_{j}}}{\prod_{j=1}^{l-1}\left(q^{2}\right)_{m_{j}}},
$$

where $\lambda_{1} \in \mathbb{Z}$ and the sum is over $m_{1}, \ldots, m_{l-1} \in \mathbb{Z}_{\geq 0}$ such that $\sum_{j=1}^{l-1} j m_{j} \equiv$ $\lambda_{1} \bmod l \mathbb{Z}$. Besides the summation condition, the RHS with the replacement $q \rightarrow q^{1 / 2}$ differs from $A_{1}^{(1)}$ case only by the factor $1 / 2$ in the quadratic form. We have

$$
\begin{aligned}
& N_{2}\left(\lambda_{1} \bar{\Lambda}_{1}, q\right) \\
& =1+2 q^{2}+4 q^{4}+8 q^{6}+15 q^{8}+26 q^{10}+44 q^{12}+72 q^{14}+115 q^{16}+\cdots \quad \text { if } \lambda_{1} \in 2 \mathbb{Z}, \\
& =q^{\frac{1}{2}}\left(1+2 q^{2}+5 q^{4}+9 q^{6}+17 q^{8}+29 q^{10}+50 q^{12}+80 q^{14}+129 q^{16}+\cdots\right) \quad \text { if } \lambda_{1} \notin 2 \mathbb{Z} . \\
& N_{3}\left(\lambda_{1} \bar{\Lambda}_{1}, q\right) \\
& =1+2 q^{2}+5 q^{4}+12 q^{6}+24 q^{8}+46 q^{10}+85 q^{12}+150 q^{14}+\cdots \quad \text { if } \lambda_{1} \in 3 \mathbb{Z}, \\
& =q^{\frac{2}{3}}\left(1+3 q^{2}+7 q^{4}+15 q^{6}+30 q^{8}+57 q^{10}+103 q^{12}+180 q^{14}+\cdots\right) \quad \text { if } \lambda_{1} \notin 3 \mathbb{Z} .
\end{aligned}
$$

Our $q$-series $N_{l}(\lambda, q)$ is connected to representation theory via 
Conjecture 5.3. For $l \in \mathbb{Z}_{\geq 1}, \lambda \in \bar{P}$ and some $\kappa \in \mathbb{Z}-\epsilon^{\prime} / a_{0}$, one has

$$
\sum_{i \in \mathbb{Z}} \operatorname{mult}_{L\left(l \Lambda_{0}\right)}\left(\lambda+l \Lambda_{0}-i \delta\right) q^{i}=q^{\kappa} N_{l}\left(\lambda, q^{\frac{1}{a_{0}}}\right) .
$$

The LHS is a string function of the level $l$ vacuum module over $X_{N}^{(r)}$ up to a power of $q$. The conjecture is valid for $A_{1}^{(1)}$ [LP, $A_{n}^{(1)}$ [FS, G, HKKOTY, and also for $l=1 \mathrm{Kad}$. For general nontwisted $\mathfrak{g}$, the $q$-series $N_{l}(\lambda, q)$ appeared in [KNS]. See also the section 6.1 of [HKKOTY] for a generalization of $N_{l}(\lambda, q)$ corresponding to the tensor product $\otimes_{i} L\left(\left(l_{i}-l_{i+1}\right) \Lambda_{0}\right)$ for nontwisted cases. It is natural to expect a similar generalization for twisted cases, but we leave it to a future study.

Under Conjecture 5.3, the property (5.3) is consistent with eq.(12.7.9) in [Kad. The asymptotic behavior of $N\left(\lambda, q^{1 / a_{0}}\right)$ as $q \rightarrow 1$ also matches the known result. To explain this let

$$
L(x)=-\frac{1}{2} \int_{0}^{x}\left(\frac{\log (1-y)}{y}+\frac{\log y}{1-y}\right) d y
$$

be the Rogers dilogarithm function. To the power series expansion $q^{-\epsilon^{\prime} / a_{0}}\left(\prod_{b=1}^{n}\left(q_{b}^{1 / a_{0}}\right)_{\infty}\right) N_{l}\left(\lambda, q^{1 / a_{0}}\right)=\sum_{k} c_{k} q^{k}$, one can apply the saddle-point method of [RS] to derive a crude estimate $\log c_{k} \sim 2 \sqrt{k a_{0} \sum_{(a, j) \in \bar{H}_{l}} L\left(f_{j}^{(a)}\right) / t_{a}^{\vee}}+$ $O(\log k)$ for $k \gg 1$. Here $f_{j}^{(a)}$ is the one in Conjecture 6.1 (iii), by which the "saddle point condition" has been solved as $q^{t_{a}^{\vee} m_{j}^{(a)} / a_{0}}=1-f_{j}^{(a)}$ with the help of 6.3). Combining the result with the well-known property $\lim _{q \rightarrow 1}(1-$ q) $\log \left(q_{a}^{1 / a_{0}}\right)_{\infty}^{-1}=\pi^{2} a_{0} /\left(6 t_{a}^{\vee}\right)$, one arrives at

$$
\lim _{q \rightarrow 1}(1-q) \log N_{l}\left(\lambda, q^{1 / a_{0}}\right)=a_{0}\left(\frac{\pi^{2}}{6} \sum_{a=1}^{n} \frac{1}{t_{a}^{\vee}}+\sum_{(a, j) \in \bar{H}_{l}} \frac{L\left(f_{j}^{(a)}\right)}{t_{a}^{\vee}}\right) .
$$

Applying (2.2) and admitting (6.4), one finally obtains

$$
\lim _{q \rightarrow 1}(1-q) \log N_{l}\left(\lambda, q^{1 / a_{0}}\right)=\frac{\pi^{2}}{6 r}\left(\frac{l \operatorname{dim} X_{N}}{l+h^{\vee}}\right) .
$$

This agrees with the behavior of the level $l$ string function as $q=\exp (-2 \pi \beta) \rightarrow 1$ given by Theorem 13.14. a) and eq.(13.13.12) in [Kad.

\section{$6 \quad Q$-system and fermionic formula at $q=1$}




\section{1 $Q$-system of type $\mathfrak{g}$}

Let $\left\{Q_{j}^{(a)} \mid 1 \leq a \leq n, j \in \mathbb{Z}_{\geq 0}\right\}$ be a set of infinitely many commutative variables with $Q_{0}^{(a)}=1$. We call the simultaneous equation

$$
Q_{j}^{(a) 2}=Q_{j+1}^{(a)} Q_{j-1}^{(a)}+Q_{j}^{(a) 2} \prod_{b=1}^{n} \prod_{k \geq 1} Q_{k}^{(b)-\left(\tilde{\alpha}_{a} \mid \tilde{\alpha}_{b}\right) B_{a j, b k} / t_{b}^{\vee}}
$$

the (unrestricted) $Q$-system of type $\mathfrak{g}$. Here $B_{a j, b k}$ is defined in $(4.22)$ and the second term on RHS actually contains finitely many factors with positive power. For $\mathfrak{g}=X_{n}^{(1)}$, it appeared in [KR2, Ki3 as a possible character identity among the irreducible finite-dimensional modules of the Yangian $Y\left(X_{n}\right)$ viewed as $X_{n}$ modules. The explicit form for the nontwisted cases is available in Appendix $\mathrm{C}$ in HKOTY1]. Let us write down the twisted cases $r=2$ and 3. Below we assume that $Q_{j}^{(a)}=1$ whenever $a \notin\{1, \ldots, n\}$.

$\mathfrak{g}=A_{2 n-1}^{(2)}:$

$$
\begin{aligned}
& Q_{j}^{(a)^{2}}=Q_{j-1}^{(a)} Q_{j+1}^{(a)}+Q_{j}^{(a-1)} Q_{j}^{(a+1)} \quad \text { for } \quad 1 \leq a \leq n-1, \\
& Q_{j}^{(n)^{2}}=Q_{j-1}^{(n)} Q_{j+1}^{(n)}+Q_{j}^{(n-1)^{2}} .
\end{aligned}
$$

$\mathfrak{g}=A_{2 n}^{(2)}:$

$$
\begin{aligned}
Q_{j}^{(a)^{2}} & =Q_{j-1}^{(a)} Q_{j+1}^{(a)}+Q_{j}^{(a-1)} Q_{j}^{(a+1)} \quad \text { for } \quad 1 \leq a \leq n-1, \\
Q_{j}^{(n)^{2}} & =Q_{j-1}^{(n)} Q_{j+1}^{(n)}+Q_{j}^{(n-1)} Q_{j}^{(n)} .
\end{aligned}
$$

$\mathfrak{g}=D_{n+1}^{(2)}:$

$$
\begin{aligned}
& Q_{j}^{(a)^{2}}=Q_{j-1}^{(a)} Q_{j+1}^{(a)}+Q_{j}^{(a-1)} Q_{j}^{(a+1)} \text { for } 1 \leq a \leq n-2, \\
& Q_{j}^{(n-1)^{2}}=Q_{j-1}^{(n-1)} Q_{j+1}^{(n-1)}+Q_{j}^{(n-2)} Q_{j}^{(n)^{2}}, \\
& Q_{j}^{(n)^{2}}=Q_{j-1}^{(n)} Q_{j+1}^{(n)}+Q_{j}^{(n-1)} .
\end{aligned}
$$

$\mathfrak{g}=E_{6}^{(2)}:$

$$
\begin{aligned}
Q_{j}^{(1)^{2}} & =Q_{j-1}^{(1)} Q_{j+1}^{(1)}+Q_{j}^{(2)}, \\
Q_{j}^{(2)^{2}} & =Q_{j-1}^{(2)} Q_{j+1}^{(2)}+Q_{j}^{(1)} Q_{j}^{(3)}, \\
Q_{j}^{(3)^{2}} & =Q_{j-1}^{(3)} Q_{j+1}^{(3)}+Q_{j}^{(2) 2} Q_{j}^{(4)}, \\
Q_{j}^{(4)^{2}} & =Q_{j-1}^{(4)} Q_{j+1}^{(4)}+Q_{j}^{(3)} .
\end{aligned}
$$

$\mathfrak{g}=D_{4}^{(3)}$ :

$$
\begin{aligned}
& Q_{j}^{(1)^{2}}=Q_{j-1}^{(1)} Q_{j+1}^{(1)}+Q_{j}^{(2)}, \\
& Q_{j}^{(2)^{2}}=Q_{j-1}^{(2)} Q_{j+1}^{(2)}+Q_{j}^{(1) 3} \text {. }
\end{aligned}
$$


Let us comment on an origin of the above proposal for twisted cases. First, it is a reduction of the $T$-system in [KS] by forgetting the spectral parameter. Second, $X_{N}^{(r)} Q$-system with $r>1$ is obtained from the $X_{N}^{(1)} Q$-system by imposing a symmetry under the order $r$ Dynkin diagram automorphism $\sigma$. To explain this, we introduce the map $\tau^{17}$ between the non 0 vertices of the Dynkin diagrams $\tau:\{1,2, \ldots, N\}$ for $X_{N}^{(1)} \rightarrow\{1, \ldots, n\}$ for $X_{N}^{(r)}$ by

$$
\begin{aligned}
& \tau(a)=\min (a, N+1-a) \quad \text { for } A_{2 n}^{(2)}, A_{2 n-1}^{(2)}, \\
& \tau(a)=\min (a, n) \quad \text { for } D_{n+1}^{(2)}, \\
& \tau(a)=\min (a, 6-a)(1 \leq a \leq 5), \tau(6)=4 \text { for } E_{6}^{(2)}, \\
& \tau(a)=1(a=1,3,4), \tau(2)=2 \text { for } D_{4}^{(3)} .
\end{aligned}
$$

The map $\tau$ respects the Dynkin diagram symmetry $\sigma$ on $\{1, \ldots, N\}$ in the sense that $\tau \circ \sigma=\tau$. Our $X_{N}^{(r)}$ type $Q$-system is obtainable from the $X_{N}^{(1)}$ type $Q$-system through the replacement $Q_{j}^{(a)} \rightarrow Q_{j}^{(\tau(a))}$.

For a given $l \in \mathbb{Z}_{\geq 1}$, impose the condition $Q_{t_{a} l}^{(a)}=1, Q_{t_{a} l+1}^{(a)}=0$. Then one can consider the truncated system of equations

$$
Q_{j}^{(a) 2}=Q_{j+1}^{(a)} Q_{j-1}^{(a)}+Q_{j}^{(a) 2} \prod_{(b, k) \in \bar{H}_{l}} Q_{k}^{(b)-\left(\tilde{\alpha}_{a} \mid \tilde{\alpha}_{b}\right) B_{a j, b k} / t_{b}^{\vee}} \quad(a, j) \in \bar{H}_{l},
$$

which close among $\left\{Q_{j}^{(a)} \mid(a, j) \in \bar{H}_{l}\right\}$. We call it the level $l$ restricted $Q$-system of type $\mathfrak{g}$. In terms of the variables $f_{j}^{(a)}=1-Q_{j+1}^{(a)} Q_{j+1}^{(a)} / Q_{j}^{(a) 2}$, it is expressed as

$$
\log f_{j}^{(a)}=\sum_{(b, k) \in \bar{H}_{l}} \frac{\left(\tilde{\alpha}_{a} \mid \tilde{\alpha}_{b}\right)}{t_{b}^{\vee}} K_{t_{b} j, t_{a} k}^{\left(t_{a} t_{b} l\right)} \log \left(1-f_{k}^{(b)}\right) .
$$

For any $\mathfrak{g}=X_{N}^{(r)}$ we have

Conjecture 6.1. (i) The unrestricted Q-system (6.1) has a solution in terms of a linear combination of $\mathfrak{\mathfrak { g }}$-characters of the form

$$
Q_{j}^{(a)}=\sum_{\lambda \in \bar{P} \cap\left(j \bar{\Lambda}_{a}-\sum_{b=1}^{n} \mathbb{Z}_{\geq 0} \alpha_{b} / \epsilon_{b}\right)} d_{\lambda} \operatorname{ch} V(\lambda), \quad d_{j \bar{\Lambda}_{a}}=1, \quad d_{\lambda} \in \mathbb{Z}_{\geq 0} .
$$

(ii) Specialization of (i) to the $q$-dimension at $q=\exp \left(2 \pi i /\left(l+h^{\vee}\right)\right)$ has the properties $Q_{t_{a} l}^{(a)}=1, Q_{t_{a} l+1}^{(a)}=0$ and $Q_{j}^{(a)} \in \mathbb{R}_{>0}$ for all $(a, j) \in \bar{H}_{l}$. (Hence it yields a solution of the level $l$ restricted $Q$-system (6.9).)

(iii) In terms of $Q_{j}^{(a)} \in \mathbb{R}_{>0}$ in (ii), set $f_{j}^{(a)}=1-Q_{j+1}^{(a)} Q_{j+1}^{(a)} / Q_{j}^{(a) 2}$. (Hence it is a solution of (6.3) such that $0<f_{j}^{(a)}<1$ for all $(a, j) \in \bar{H}_{l}$.) Then we have

$$
a_{0} r \sum_{(a, j) \in \bar{H}_{l}} \frac{L\left(f_{j}^{(a)}\right)}{t_{a}^{\vee}}=\frac{\pi^{2}}{6}\left(\frac{l \operatorname{dim} X_{N}}{l+h^{\vee}}-N\right)
$$

\footnotetext{
${ }^{17}$ The $\tau$ here should be distinguished from the one in 3.3 .
} 
Several remarks are in order. First, (i) is indeed valid if $\mathfrak{g}$ is non-exceptional or $D_{4}^{(3)}$. The explicit forms are available in KR2, HKOTY1 for the nontwisted cases $\mathfrak{g}=A_{n}^{(1)}, B_{n}^{(1)}, C_{n}^{(1)}, D_{n}^{(1)}$ and in Section 6.2 of this paper for $\mathfrak{g}=A_{2 n}^{(2)}, A_{2 n-1}^{(2)}, D_{n+1}^{(2)}$ and $D_{4}^{(3)}$. Thus (i) is actually an existence conjecture for the other $\mathfrak{g}$ 's. On the other hand for any $\mathfrak{g}$, the forthcoming Theorem 6.3 asserts the uniqueness as well as a unified construction $Q_{j}^{(a)}=\sum_{\lambda} \tilde{M}\left(W_{j}^{(a)}, \lambda, 1\right) \operatorname{ch} V(\lambda)$ in terms of the fermionic form at $q=1$ under a further assumption (C) on the asymptotic behavior. For the exceptional $\mathfrak{g}$ 's other than $D_{4}^{(3)}$, it is yet to be confirmed if the above $Q_{j}^{(a)}$ indeed satisfies the $Q$-system. Second, if Conjecture 5.3 and 6.1 (i)-(ii) are valid, then the dilogarithm sum rule (6.4) follows from (5.5) and Theorem 13.14. a) in [Kad by reversing the argument in the end of Section 5. In this sense Conjecture 5.3 is a $q$-analogue of (6.4). Third, (6.4) with $r=1$ was originally conjectured in [Ki3], where $A_{n}^{(1)}$ case was proved. (For $X_{n}^{(1)}$ non-simply laced, the range of the sum therein is misprinted and should be read as above.) Our (6.4) for $X_{N}^{(r)}$ with $r>1$ is actually a consequence of the $X_{N}^{(1)}$ case. To see this, note that $f_{j}^{\prime(a)}$ for $X_{N}^{(1)}$ enjoys the symmetry $f_{j}^{\prime(a)}=f_{j}^{\prime(\sigma(a))}$ under the order $r$ Dynkin diagram automorphism $\sigma$. Therefore $f_{j}^{(a)}$ for $X_{N}^{(r)}$ is obtained as $f_{j}^{(a)}=f_{j}^{\prime(b)}$ for any $b$ such that $\tau(b)=a$. In view of this, LHS of (6.4) is equal to $\sum_{a=1}^{N} \sum_{j=1}^{l-1} L\left(f_{j}^{\prime(a)}\right)$, reducing it to the $X_{N}^{(1)}$ case since $h^{\vee}$ is independent of $r$. In particular, the factor $a_{0} r / t_{a}^{\vee} \in\{1, r\}$ has the interpretation

$$
\frac{a_{0} r}{t_{a}^{\vee}}=\sharp\{b \in\{1, \ldots, N\} \mid \tau(b)=a\} \quad \text { for } 1 \leq a \leq n .
$$

In view of (2.2) and $f_{t_{a} l}^{(a)}=1$ under $Q_{t_{a} l+1}^{(a)}=0$, the sum rule (6.4) is also stated as

$$
a_{0} r \sum_{(a, j) \in H_{l}} \frac{L\left(f_{j}^{(a)}\right)}{t_{a}^{\vee}}=\frac{\pi^{2}}{6}\left(\frac{l \operatorname{dim} X_{N}}{l+h^{\vee}}\right) .
$$

\subsection{Solutions in terms of classical characters}

Let us present solutions of the unrestricted $Q$-system (6.1) for $\mathfrak{g}=A_{2 n}^{(2)}, A_{2 n-1}^{(2)}$, $D_{n+1}^{(2)}, D_{4}^{(3)}$ in terms of the character of a classical subalgebra $\mathfrak{g}^{\prime} \subset \mathfrak{g}$. Similar result is available for $\mathfrak{g}=A_{n}^{(1)}, B_{n}^{(1)}, C_{n}^{(1)}, D_{n}^{(1)}$ KR2, HKOTY1 for the choice $\mathfrak{g}^{\prime}=$ $\mathfrak{g}$. Here we shall include the result $\left(\mathfrak{g}, \mathfrak{g}^{\prime}\right)=\left(A_{2 n}^{(2)}, C_{n}\right),\left(A_{2 n}^{(2)}, B_{n}\right),\left(A_{2 n-1}^{(2)}, C_{n}\right)$, $\left(A_{2 n-1}^{(2)}, D_{n}\right),\left(D_{n+1}^{(2)}, B_{n}\right)$ and $\left(D_{4}^{(3)}, G_{2}\right)$. In case $\mathfrak{g}^{\prime}=\stackrel{\circ}{\mathfrak{g}}$, Appendix $\mathrm{A}$ with $q=1$ yields further conjectures on $E_{6}^{(2)}$.

Let us introduce the linear combination of characters. $\left(\mathfrak{g}, \mathfrak{g}^{\prime}\right)=\left(A_{2 n}^{(2)}, C_{n}\right)$ :

$$
\chi_{j}^{(a)}=\sum \operatorname{ch} V\left(k_{1} \bar{\Lambda}_{1}^{\prime}+k_{2} \bar{\Lambda}_{2}^{\prime}+\cdots+k_{a} \bar{\Lambda}_{a}^{\prime}\right) \quad \text { for } \quad 1 \leq a \leq n .
$$


$\left(\mathfrak{g}, \mathfrak{g}^{\prime}\right)=\left(A_{2 n}^{(2)}, B_{n}\right):$

$$
\chi_{j}^{(a)}=\left\{\begin{array}{cl}
\sum \operatorname{ch} V\left(k_{1} \bar{\Lambda}_{1}^{\prime}+k_{2} \bar{\Lambda}_{2}^{\prime}+\cdots+k_{a} \bar{\Lambda}_{a}^{\prime}\right) & \text { if } \quad 1 \leq a \leq n-1, \\
\sum \operatorname{ch} V\left(k_{1} \bar{\Lambda}_{1}^{\prime}+k_{2} \bar{\Lambda}_{2}^{\prime}+\cdots+k_{n-1} \bar{\Lambda}_{n-1}^{\prime}\right. & \\
\left.+2 k_{n} \bar{\Lambda}_{n}^{\prime}\right) & \text { if } \quad a=n .
\end{array}\right.
$$

$\left(\mathfrak{g}, \mathfrak{g}^{\prime}\right)=\left(A_{2 n-1}^{(2)}, C_{n}\right):$

$$
\begin{aligned}
\chi_{j}^{(a)}=\sum \operatorname{ch} V\left(k_{b} \bar{\Lambda}_{b}^{\prime}+k_{b+2} \bar{\Lambda}_{b+2}^{\prime}+\cdots+k_{a} \bar{\Lambda}_{a}^{\prime}\right) \\
\text { for } 1 \leq a \leq n, \quad b \equiv a \bmod 2, \quad b=0 \text { or } 1 .
\end{aligned}
$$

$\left(\mathfrak{g}, \mathfrak{g}^{\prime}\right)=\left(A_{2 n-1}^{(2)}, D_{n}\right):$

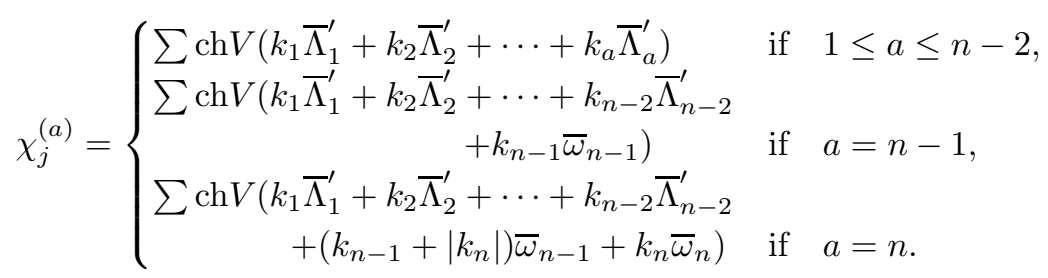

$\left(\mathfrak{g}, \mathfrak{g}^{\prime}\right)=\left(D_{n+1}^{(2)}, B_{n}\right):$

$$
\chi_{j}^{(a)}=\left\{\begin{array}{lll}
\sum \operatorname{ch} V\left(k_{1} \bar{\Lambda}_{1}^{\prime}+k_{2} \bar{\Lambda}_{2}^{\prime}+\cdots+k_{a} \bar{\Lambda}_{a}^{\prime}\right) & \text { if } 1 \leq a \leq n-1, \\
\operatorname{ch} V\left(j \bar{\Lambda}_{n}^{\prime}\right) & \text { if } \quad a=n .
\end{array}\right.
$$

$\left(\mathfrak{g}, \mathfrak{g}^{\prime}\right)=\left(D_{4}^{(3)}, G_{2}\right):$

$$
\begin{aligned}
& \chi_{j}^{(1)}=\sum_{k=0}^{j} \operatorname{ch} V\left(k \bar{\Lambda}_{1}^{\prime}\right), \\
& \chi_{j}^{(2)}=\sum_{\substack{k_{1}+k_{2} \leq j \\
k_{1}, k_{2} \in \mathbb{Z}_{\geq 0}}} \min \left(1+k_{2}, 1+j-k_{1}-k_{2}\right)\left(k_{1}+1\right) \operatorname{ch} V\left(k_{1} \bar{\Lambda}_{1}^{\prime}+k_{2} \bar{\Lambda}_{2}^{\prime}\right) .
\end{aligned}
$$

Here $\operatorname{ch} V(\lambda)$ denotes the irreducible $\mathfrak{g}^{\prime}$ character with highest weight $\lambda . \bar{\Lambda}_{a}^{\prime}(1 \leq$ $a \leq n)$ are the fundamental weights of $\mathfrak{g}^{\prime}$. We have also used the convention $\bar{\Lambda}_{0}^{\prime}=0$ and $\bar{\omega}_{n-1}=\bar{\Lambda}_{n-1}^{\prime}+\bar{\Lambda}_{n}^{\prime}, \bar{\omega}_{n}=-\bar{\Lambda}_{n-1}^{\prime}+\bar{\Lambda}_{n}^{\prime}$. The sums in $6.6-6.10$ are taken as follows.

(6.6), 6.10) $)_{a<n}: k_{1}, \ldots, k_{a} \in \mathbb{Z}_{\geq 0}, k_{1}+\cdots+k_{a} \leq j$,

$$
\text { (6.8) }: k_{b}, k_{b+2}, \ldots, k_{a} \in \mathbb{Z}_{\geq 0}, k_{b}+k_{b+2}+\cdots+k_{a}=j,
$$

(6.7), (6.9) $)_{a<n}: k_{1}, \ldots, k_{a} \in \mathbb{Z}_{\geq 0}, k_{1}+\cdots+k_{a} \leq j, k_{b}-j \delta_{a b} \in 2 \mathbb{Z}(1 \leq \forall b \leq a)$,

$$
\begin{aligned}
(\text { 6.9 })_{a=n}: & k_{1}, \ldots, k_{n-1} \in \mathbb{Z}_{\geq 0}, k_{n} \in \mathbb{Z}, k_{1}+\cdots+k_{n-1}+\left|k_{n}\right| \leq j, \\
& k_{b}-j \delta_{n b} \in 2 \mathbb{Z}(1 \leq \forall b \leq n) .
\end{aligned}
$$


If one depicts the highest weights in the sums (6.8), $\left.((6.6),(6.10))_{a<n}\right)$ and $\left.(\sqrt{6.7})_{a<n},(6.9)_{a<n-1}\right)$ with the Young diagrams as usual, they correspond to those obtained from the $a \times j$ rectangle by successively removing $2 \times 1,1 \times 1$ and $1 \times 2$ blocks, respectively.

Let $x_{a}=e^{\bar{\Lambda}_{a}^{\prime}}$ be a complex variable. Then the $\mathfrak{g}^{\prime}$ character $\operatorname{ch} V(\lambda)$ belongs to $\mathbb{Z}\left[x_{1}^{ \pm 1}, \ldots, x_{n}^{ \pm 1}\right]$. By an argument similar to [HKOTY1] one can show

Theorem 6.2. If $\left(\mathfrak{g}, \mathfrak{g}^{\prime}\right)=\left(A_{2 n-1}^{(2)}, C_{n}\right),\left(A_{2 n}^{(2)}, C_{n}\right),\left(D_{n+1}^{(2)}, B_{n}\right)$ or $\left(D_{4}^{(3)}, G_{2}\right)$ (i.e., $\mathfrak{g}^{\prime}=\stackrel{\circ}{\mathfrak{g}}$ hence $\bar{\Lambda}_{a}^{\prime}=\bar{\Lambda}_{a}$ ), we have
(A) $\chi_{j}^{(a)}=\sum_{\lambda \in \bar{P}^{+}} d_{\lambda} \operatorname{ch} V(\lambda), d_{\lambda} \in \mathbb{C}, d_{j \bar{\Lambda}_{a}}=1, d_{\lambda}=0$ unless $\lambda \in j \bar{\Lambda}_{a}-$ $\sum_{b=1}^{n} \mathbb{Z}_{\geq 0} \alpha_{b} / \epsilon_{b}, \quad 1 \leq a \leq n, j \in \mathbb{Z}_{\geq 0}$,
(B) $Q_{j}^{(a)}=\chi_{j}^{(a)}$ solves the Q-system (6.1),
(C) $\lim _{j \rightarrow \infty}\left(\frac{\chi_{j}^{(a)}}{\chi_{j+1}^{(a)}}\right)=x_{a}^{-1}$ in the domain $\left|e^{\alpha_{1}}\right|, \ldots,\left|e^{\alpha_{n}}\right|>1$.

When $\left(\mathfrak{g}, \mathfrak{g}^{\prime}\right)=\left(A_{2 n-1}^{(2)}, D_{n}\right)$ or $\left(A_{2 n}^{(2)}, B_{n}\right),(\mathrm{B})$ is still valid for $\chi_{j}^{(a)}$ given in (6.7) or (6.9). However, (A) and (C) no longer hold. For example instead of (C), one has

$$
\begin{aligned}
& \left(\mathfrak{g}, \mathfrak{g}^{\prime}\right)=\left(A_{2 n-1}^{(2)}, D_{n}\right): \\
& \lim _{j \rightarrow \infty}\left(\frac{\chi_{j}^{(a)}}{\chi_{j+1}^{(a)}}\right)= \begin{cases}x_{a}^{-1} & \text { if } 1 \leq a \leq n-2, \\
\left(x_{n-1} x_{n}\right)^{-1} & \text { if } a=n-1, \\
x_{n-1}^{-2} & \text { if } a=n \text { and }\left|e^{\alpha_{n-1}^{\prime}}\right|>\left|e^{\alpha_{n}^{\prime}}\right|, \\
x_{n}^{-2} & \text { if } a=n \text { and }\left|e^{\alpha_{n-1}^{\prime}}\right|<\left|e^{\alpha_{n}^{\prime}}\right|,\end{cases}
\end{aligned}
$$

$\left(\mathfrak{g}, \mathfrak{g}^{\prime}\right)=\left(A_{2 n}^{(2)}, B_{n}\right):$

$$
\lim _{j \rightarrow \infty}\left(\frac{\chi_{j}^{(a)}}{\chi_{j+1}^{(a)}}\right)=\left\{\begin{array}{lll}
x_{a}^{-1} & \text { if } & 1 \leq a \leq n-1, \\
x_{n}^{-2} & \text { if } & a=n
\end{array}\right.
$$

in the domain $\left|e^{\alpha_{1}^{\prime}}\right|, \ldots,\left|e^{\alpha_{n}^{\prime}}\right|>1$, where $\alpha_{a}^{\prime}$ denotes a simple root of $\mathfrak{g}^{\prime}$.

The fact that the $\mathfrak{g}^{\prime}$ character $\chi_{j}^{(a)}$ satisfies the $Q$-system of type $\mathfrak{g}=X_{N}^{(2)}$ can be shown by using the solution to the nontwisted cases $X_{N}^{(1)}$ KR2, HKOTY1. All one needs is to restrict the earlier solution in terms of $X_{N \text {-characters to }}$ those for the subalgebras $\mathfrak{g}^{\prime}$. It is done by means of a class of identities known as Littlewood's lemma $[\mathbb{4}$. See for example the references listed in the sequel. $\left(\mathfrak{g}, \mathfrak{g}^{\prime}\right)=\left(A_{2 n-1}^{(2)}, C_{n}\right):$ Theorem in [KS] and eq. (1) in p.492 (or eq. (2) in p.507) of [KT].

$\left(\mathfrak{g}, \mathfrak{g}^{\prime}\right)=\left(A_{2 n-1}^{(2)}, D_{n}\right)$ : Theorem in $[\mathrm{KS}$, eq. (1.1) in p.471 of [KT and eq. (5) in p.492 (or eq. (3) in p.507) of KT].

$\left(\mathfrak{g}, \mathfrak{g}^{\prime}\right)=\left(A_{2 n}^{(2)}, C_{n}\right)$ : Theorem in [KS] and eq. (1.5.1) in p.488 of [KT], the 
equation under eq. (1.5.2) in p.490 of [KT] and eq. $(11.9 ; 6)$ in p.238 of [L].

$\left(\mathfrak{g}, \mathfrak{g}^{\prime}\right)=\left(A_{2 n}^{(2)}, B_{n}\right)$ : Theorem in $\mathrm{KS}$, the equation under eq. (1.1) in p.471 of KT and eq. (3) in p.492 (or eq.(1) in p.506) of [KT.

$\left(\mathfrak{g}, \mathfrak{g}^{\prime}\right)=\left(D_{n+1}^{(2)}, B_{n}\right)$ : Theorem 7.1 (B) for $D_{n+1}^{(1)}$ in HKOTY1 and a branching rule for $O(2 n+2) \downarrow O(2 n+1)[\mathrm{W}]$ and $O(n) \downarrow S O(n)(\mathrm{p} .471$ of [KT]).

$\left(\mathfrak{g}, \mathfrak{g}^{\prime}\right)=\left(D_{4}^{(3)}, G_{2}\right)$ : Theorem 7.1 (B) for $D_{4}^{(1)}$ in HKOTY1 and the branching rules $O(8) \downarrow O(7)[\mathrm{W}]$ and $O(7) \downarrow G_{2}[\mathrm{Wy}]$.

The solution (6.7) of the $A_{2 n}^{(2)}$ type $Q$-system is relevant to a superalgebra $B^{(1)}(0 \mid s)$ 田. In Appendix $\mathrm{Q}$ we have also included a solution of $B_{n}^{(1)} Q$-system in terms of $D_{n}$ characters.

\subsection{Fermionic form and $Q$-system}

In this subsection we consider the pair $\mathfrak{g} \subset \mathfrak{g}$ for general $\mathfrak{g}$.

Theorem 6.3. Suppose that a linear combination of $\stackrel{\circ}{\mathfrak{g}}$ characters

$$
Q_{j}^{(a)}=\sum_{\lambda \in \bar{P}^{+}} d_{\lambda} \operatorname{ch} V(\lambda) \quad 1 \leq a \leq n, j \in \mathbb{Z}_{\geq 0}
$$

possesses the properties:

(A) $d_{\lambda} \in \mathbb{C}, d_{j \bar{\Lambda}_{a}}=1, d_{\lambda}=0$ unless $\lambda \in j \bar{\Lambda}_{a}-\sum_{b=1}^{n} \mathbb{Z}_{\geq 0} \alpha_{b} / \epsilon_{b}$,

(B) $\left\{Q_{j}^{(a)}\right\}$ satisfies the $Q$-system (6.1),

(C) $\lim _{j \rightarrow \infty}\left(\frac{Q_{j}^{(a)}}{Q_{j+1}^{(a)}}\right)=e^{-\bar{\Lambda}_{a}}$ in the domain $\left|e^{\alpha_{1}}\right|, \ldots,\left|e^{\alpha_{n}}\right|>1$.

Then for any finitely many non-negative integers $\nu_{j}^{(a)}$ we have

$$
\prod_{a=1}^{n} \prod_{j \geq 1}\left(Q_{j}^{(a)}\right)^{\nu_{j}^{(a)}}=\sum_{\lambda} \tilde{M}_{\infty}(W, \lambda, 1) \operatorname{ch} V(\lambda),
$$

where $\tilde{M}_{\infty}(W, \lambda, 1)$ is defined by (4.20) with $W=\bigotimes_{a=1}^{n} \bigotimes_{j \geq 1}\left(W_{j}^{(a)}\right)^{\otimes \nu_{j}^{(a)}}$. The sum $\sum_{\lambda}$ runs over $\lambda \in\left(\sum_{a=1}^{n} \sum_{i \geq 1} i \nu_{i}^{(a)} \bar{\Lambda}_{a}-\sum_{b=1}^{n} \mathbb{Z}_{\geq 0} \alpha_{b} / \epsilon_{b}\right) \cap \bar{P}^{+}$.

This theorem has analogous aspects to Theorem 8.1 in HKOTY1, where $r=$ 1 case was treated. In particular it asserts the uniqueness of $\left\{Q_{j}^{(a)}\right\}$ satisfying (A) - (C), but does not assure the existence, i.e. it does not guarantee that the functions $\left\{\sum_{\lambda} \tilde{M}_{\infty}\left(W_{j}^{(a)}, \lambda, 1\right) \operatorname{ch} V(\lambda)\right\}$ fulfill (B) and (C) In this paper, we have shown the existence of such $\left\{Q_{j}^{(a)}\right\}$ for $\mathfrak{g}=A_{2 n-1}^{(2)}, A_{2 n}^{(2)}, D_{n+1}^{(2)}$ and $D_{4}^{(3)}$ in Theorem 6.2.

\footnotetext{
${ }^{18}$ They do (A). Note also the consistency of the condition (B) with Proposition 4.4 at $q=1$.
} 
We omit the proof of Theorem 6.3 because it is essentially a repetition of the one in HKOTY1], whose idea goes back to Ki1, Ki2]. Another reason is that it is a corollary of more general results in [KN, KNT]. In these works, power series solutions of a generalized $Q$-system adapted to $e^{-j \bar{\Lambda}_{a}} Q_{j}^{(a)}$ has been studied. A proof of uniqueness, existence and two explicit constructions have been done under a certain convergence condition. (6.14) follows from them upon a further assumption of the Weyl group invariance.

For nontwisted cases $r=1$, the identity (6.14) can be interpreted as a formal (combinatorial) completeness of the Bethe ansatz for rational vertex models with Yangian $Y\left(X_{N}\right)$-symmetry [KR2, Ki1, Ki2. They correspond to $q \rightarrow 1$ degeneration of trigonometric vertex models with $U_{q}^{\prime}\left(X_{N}^{(1)}\right)$ symmetry, and the fermionic form $\tilde{M}_{\infty}(W, \lambda, 1)$ arises naturally from the rational Bethe equation [DW] under a string hypothesis. It is tempting to interpret (6.14) similarly for

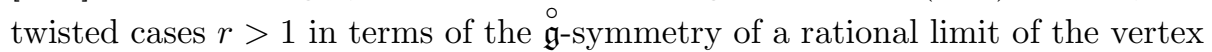
models associated with the twisted $U_{q}^{\prime}\left(X_{N}^{(r)}\right)$. However, at present we do not know a way, even formally, to derive $\tilde{M}_{\infty}(W, \lambda, 1)$ with $r>1$ from a rational limit of the Bethe equation [RW] and a string hypothesis. We remark that in a naive $q \rightarrow 1$ limit, transfer matrix spectrum turns out to exhibit a much larger

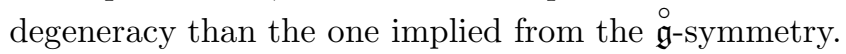

\section{A List of $M_{\infty}\left(W_{s}^{(a)}, \lambda, q^{-1}\right)$}

Consider a formal linear combination of the $\stackrel{\circ}{\mathfrak{g} \text { modules }} V(\lambda)$ of highest weight $\lambda$ :

$$
\mathcal{W}_{s}^{(a)}=\sum_{\lambda \in \bar{P}^{+}} M_{\infty}\left(W_{s}^{(a)}, \lambda, q^{-1}\right) V(\lambda) .
$$

In this appendix, we give a list of $\mathcal{W}_{s}^{(a)}$ for $\mathfrak{g}=A_{2 n-1}^{(2)}, A_{2 n}^{(2)}, D_{n+1}^{(2)}$ and a conjecture (in some cases) for $\mathfrak{g}=E_{6}^{(2)}, D_{4}^{(3)}$. When $q=1$ they reduce to 6.6), (6.8) and (6.10) for $A_{2 n}^{(2)}, A_{2 n-1}^{(2)}$ and $D_{n+1}^{(2)}$, respectively. $\mathfrak{g}=A_{2 n-1}^{(2)}$ :

$$
\mathcal{W}_{s}^{(a)}=\sum_{\lambda} q^{\frac{1}{2}\left(\bar{\Lambda}_{n} \mid s \bar{\Lambda}_{a}-\lambda\right)} V(\lambda) \quad(1 \leq a \leq n),
$$

where the sum $\sum_{\lambda}$ is taken over $\lambda \in\left\{k_{e} \bar{\Lambda}_{e}+k_{e+2} \bar{\Lambda}_{e+2}+\cdots+k_{a} \bar{\Lambda}_{a} \in \bar{P}^{+} \mid k_{e}+\right.$ $\left.k_{e+2}+\cdots+k_{a-2}+k_{a}=s\right\}$ with $\bar{\Lambda}_{0}=0$ and $e \equiv a(\bmod 2), e=0$ or 1 . In the computation of $M_{\infty}\left(W_{s}^{(a)}, k_{e} \bar{\Lambda}_{e}+k_{e+2} \bar{\Lambda}_{e+2}+\cdots+k_{a} \bar{\Lambda}_{a}, q^{-1}\right)$, the only choice of $\left\{m_{j}^{(c)}\right\}$ such that $\forall p_{i}^{(c)} \geq 0$ is the following:

if $a=2 u$ is even, then

$$
\left\{\begin{array}{ll}
m_{j}^{(2 c-1)}=\sum_{b=1}^{\min (c-1, u)} \delta_{j, l_{b}}+\sum_{b=1}^{\min (c, u)} \delta_{j, l_{b}} & (c \geq 1,2 c-1 \leq n-1, j \geq 1) \\
m_{j}^{(2 c)}=2 \sum_{b=1}^{\min (c, u)} \delta_{j, l_{b}} & (c \geq 1,2 c \leq n-1, j \geq 1) \\
m_{j}^{(n)}=\sum_{b=1}^{u} \delta_{j, l_{b}} & (j \geq 1)
\end{array},\right.
$$


and if $a=2 u+1$ is odd, then

$$
\begin{cases}m_{j}^{(1)}=0 & (j \geq 1) \\ m_{j}^{(2 c)}=\sum_{b=1}^{\min (c-1, u)} \delta_{j, l_{b}}+\sum_{b=1}^{\min (c, u)} \delta_{j, l_{b}} & (c \geq 1,2 c \leq n-1, j \geq 1) \\ m_{j}^{(2 c+1)}=2 \sum_{b=1}^{\min (c, u)} \delta_{j, l_{b}} & (c \geq 1,2 c+1 \leq n-1, j \geq 1) \\ m_{j}^{(n)}=\sum_{b=1}^{u} \delta_{j, l_{b}} & (j \geq 1)\end{cases}
$$

where $l_{b}=s-\left(k_{a}+k_{a-2}+\cdots+k_{2 b+e}\right)$ for the both cases.

$\mathfrak{g}=A_{2 n}^{(2)}$ :

$$
\mathcal{W}_{s}^{(a)}=\sum_{\lambda} q^{\left(\bar{\Lambda}_{n} \mid s \bar{\Lambda}_{a}-\lambda\right)} V(\lambda) \quad(1 \leq a \leq n)
$$

where the sum $\sum_{\lambda}$ is taken over $\lambda \in\left\{k_{1} \bar{\Lambda}_{1}+\cdots+k_{a} \bar{\Lambda}_{a} \in \bar{P}^{+} \mid k_{1}+\cdots+k_{a} \leq s\right\}$. In the computation of $M_{\infty}\left(W_{s}^{(a)}, k_{1} \bar{\Lambda}_{1}+\cdots+k_{a} \bar{\Lambda}_{a}, q^{-1}\right)$, the only choice of $\left\{m_{j}^{(c)}\right\}$ such that $\forall p_{i}^{(c)} \geq 0$ is the following:

$$
m_{j}^{(c)}=\sum_{b=1}^{\min (c, a)} \delta_{j, l_{b}} \quad(1 \leq c \leq n, j \geq 1),
$$

where $l_{b}=s-\left(k_{b}+k_{b+1}+\cdots+k_{a}\right)$.

$\mathfrak{g}=D_{n+1}^{(2)}:$

$$
\mathcal{W}_{s}^{(a)}=\left\{\begin{array}{ll}
\sum_{\lambda} q^{\left(\bar{\Lambda}_{n} \mid s \bar{\Lambda}_{a}-\lambda\right)} V(\lambda) & (1 \leq a \leq n-1) \\
V\left(s \bar{\Lambda}_{n}\right) & (a=n)
\end{array},\right.
$$

where the sum $\sum_{\lambda}$ is taken over $\lambda \in\left\{k_{1} \bar{\Lambda}_{1}+\cdots+k_{a} \bar{\Lambda}_{a} \in \bar{P}^{+} \mid k_{1}+\cdots+k_{a} \leq s\right\}$. In the computation of $M_{\infty}\left(W_{s}^{(a)}, k_{1} \bar{\Lambda}_{1}+\cdots+k_{a} \bar{\Lambda}_{a}, q^{-1}\right)$, the only choice of $\left\{m_{j}^{(c)}\right\}$ such that $\forall p_{i}^{(c)} \geq 0$ is the same as $\mathfrak{g}=A_{2 n}^{(2)}$ case. 


$$
\begin{aligned}
\mathfrak{g}=E_{6}^{(2)}: & \\
\mathcal{W}_{1}^{(1)}= & q V(0)+V\left(\bar{\Lambda}_{1}\right) \\
\mathcal{W}_{1}^{(2)}= & q^{3} V(0)+\left(q+q^{2}\right) V\left(\bar{\Lambda}_{1}\right)+q V\left(\bar{\Lambda}_{4}\right)+V\left(\bar{\Lambda}_{2}\right), \\
\mathcal{W}_{1}^{(3)}= & \left(q^{4}+q^{6}\right) V(0)+\left(2 q^{3}+q^{4}+q^{5}\right) V\left(\bar{\Lambda}_{1}\right)+\left(2 q^{2}+q^{4}\right) V\left(\bar{\Lambda}_{4}\right) \\
& +\left(q+q^{2}+q^{3}\right) V\left(\bar{\Lambda}_{2}\right)+q^{2} V\left(2 \bar{\Lambda}_{1}\right)+q V\left(\bar{\Lambda}_{1}+\bar{\Lambda}_{4}\right)+V\left(\bar{\Lambda}_{3}\right), \\
\mathcal{W}_{2}^{(3)}= & \left(q^{8}+q^{10}+q^{12}\right) V(0)+\left(2 q^{6}+4 q^{8}+q^{10}\right) V\left(\bar{\Lambda}_{4}\right) \\
& +\left(3 q^{4}+3 q^{6}+q^{8}\right) V\left(2 \bar{\Lambda}_{4}\right)+\left(2 q^{4}+q^{5}+6 q^{6}+q^{7}+q^{8}\right) V\left(\bar{\Lambda}_{3}\right) \\
& +\left(2 q^{2}+2 q^{4}\right) V\left(\bar{\Lambda}_{3}+\bar{\Lambda}_{4}\right)+V\left(2 \bar{\Lambda}_{3}\right) \\
& +\left(q^{5}+2 q^{6}+5 q^{7}+3 q^{8}+2 q^{9}\right) V\left(\bar{\Lambda}_{2}\right) \\
& +\left(2 q^{3}+3 q^{4}+5 q^{5}+2 q^{6}+q^{7}\right) V\left(\bar{\Lambda}_{2}+\bar{\Lambda}_{4}\right) \\
& +\left(q+q^{2}+q^{3}\right) V\left(\bar{\Lambda}_{2}+\bar{\Lambda}_{3}\right)+\left(q^{2}+q^{3}+3 q^{4}+q^{5}+q^{6}\right) V\left(2 \bar{\Lambda}_{2}\right) \\
& +\left(2 q^{7}+q^{8}+3 q^{9}+q^{10}+q^{11}\right) V\left(\bar{\Lambda}_{1}\right) \\
& +\left(5 q^{5}+3 q^{6}+6 q^{7}+q^{8}+q^{9}\right) V\left(\bar{\Lambda}_{1}+\bar{\Lambda}_{4}\right) \\
& +\left(2 q^{3}+q^{5}\right) V\left(\bar{\Lambda}_{1}+2 \bar{\Lambda}_{4}\right)+\left(3 q^{3}+2 q^{4}+3 q^{5}\right) V\left(\bar{\Lambda}_{1}+\bar{\Lambda}_{3}\right) \\
& +q V\left(\bar{\Lambda}_{1}+\bar{\Lambda}_{3}+\bar{\Lambda}_{4}\right)+\left(2 q^{4}+5 q^{5}+5 q^{6}+3 q^{7}+q^{8}\right) V\left(\bar{\Lambda}_{1}+\bar{\Lambda}_{2}\right) \\
& +\left(q^{2}+2 q^{3}+q^{4}\right) V\left(\bar{\Lambda}_{1}+\bar{\Lambda}_{2}+\bar{\Lambda}_{4}\right) \\
& +\left(4 q^{6}+2 q^{7}+4 q^{8}+q^{9}+q^{10}\right) V\left(2 \bar{\Lambda}_{1}\right) \\
& +\left(4 q^{4}+q^{5}+2 q^{6}\right) V\left(2 \bar{\Lambda}_{1}+\bar{\Lambda}_{4}\right)+q^{2} V\left(2 \bar{\Lambda}_{1}+2 \bar{\Lambda}_{4}\right)+q^{2} V\left(2 \bar{\Lambda}_{1}+\bar{\Lambda}_{3}\right) \\
& +\left(q^{3}+q^{4}+q^{5}\right) V\left(2 \bar{\Lambda}_{1}+\bar{\Lambda}_{2}\right)+\left(2 q^{5}+q^{6}+q^{7}\right) V\left(3 \bar{\Lambda}_{1}\right) \\
& +q^{3} V\left(3 \bar{\Lambda}_{1}+\bar{\Lambda}_{4}\right)+q^{4} V\left(4 \bar{\Lambda}_{1}\right), \\
\mathcal{W}_{1}^{(4)}= & q^{2} V(0)+q V\left(\bar{\Lambda}_{1}\right)+V\left(\bar{\Lambda}_{4}\right) . \\
&
\end{aligned}
$$

In addition we have a conjecture for $\mathcal{W}_{s}^{(a)}(a=1,2,4)$ :

$$
\begin{aligned}
\mathcal{W}_{s}^{(1)}= & \sum_{k=0}^{s} q^{s-k} V\left(k \bar{\Lambda}_{1}\right) \\
\mathcal{W}_{s}^{(2)}= & \sum_{\substack{j_{1}+j_{2}+2 j_{3}+j_{4} \leq s \\
j_{1}, j_{2}, j_{3}, j_{4} \in \mathbb{Z} \geq 0}} \min \left(1+j_{2}, 1+s-j_{1}-j_{2}-2 j_{3}-j_{4}\right) q^{3 s-2 j_{1}-3 j_{2}-4 j_{3}-2 j_{4}} \\
& \times\left[\begin{array}{c}
j_{1}+1 \\
1
\end{array}\right]_{q} V\left(j_{1} \bar{\Lambda}_{1}+j_{2} \bar{\Lambda}_{2}+j_{3} \bar{\Lambda}_{3}+j_{4} \bar{\Lambda}_{4}\right) \\
\mathcal{W}_{s}^{(4)}= & \sum_{\substack{j_{1}+j_{4} \leq s \\
j_{1}, j_{4} \in \mathbb{Z}_{\geq 0}}} q^{2 s-j_{1}-2 j_{4}} V\left(j_{1} \bar{\Lambda}_{1}+j_{4} \bar{\Lambda}_{4}\right)
\end{aligned}
$$

The conjecture has been checked for $1 \leq s \leq 8$. In addition, we have observed that the support for $V\left(j_{1} \bar{\Lambda}_{1}+j_{2} \bar{\Lambda}_{2}+j_{3} \bar{\Lambda}_{3}+j_{4} \bar{\Lambda}_{4}\right)$ in $\mathcal{W}_{s}^{(3)}$ is given by the condition $j_{1}+2 j_{2}+2 j_{3}+j_{4} \leq 2 s,\left[\left(j_{2}+1\right) / 2\right]+j_{3}+j_{4} \leq s, j_{1}, j_{2}, j_{3}, j_{4} \in \mathbb{Z}_{\geq 0}$ 
at least for $1 \leq s \leq 7$ 19.

$\mathfrak{g}=D_{4}^{(3)}:$

$$
\begin{aligned}
& \mathcal{W}_{1}^{(1)}=q V(0)+V\left(\bar{\Lambda}_{1}\right), \\
& \mathcal{W}_{1}^{(2)}=q^{3} V(0)+\left(q+q^{2}\right) V\left(\bar{\Lambda}_{1}\right)+V\left(\bar{\Lambda}_{2}\right) .
\end{aligned}
$$

In addition we have a conjecture for $\mathcal{W}_{s}^{(a)}$ :

$$
\begin{aligned}
\mathcal{W}_{s}^{(1)}= & \sum_{k=0}^{s} q^{s-k} V\left(k \bar{\Lambda}_{1}\right) \\
\mathcal{W}_{s}^{(2)}= & \sum_{\substack{j_{1}+j_{2} \leq s \\
j_{1}, j_{2} \in \mathbb{Z}_{\geq 0}}} \min \left(1+j_{2}, 1+s-j_{1}-j_{2}\right) q^{3 s-2 j_{1}-3 j_{2}} \\
& \times\left[\begin{array}{c}
j_{1}+1 \\
1
\end{array}\right]_{q} V\left(j_{1} \bar{\Lambda}_{1}+j_{2} \bar{\Lambda}_{2}\right) .
\end{aligned}
$$

The conjecture has been checked for $1 \leq s \leq 20$. This is consistent with the $q=1$ result $(6.11$.

\section{B Explicit form of Proposition 4.10}

Let us write down 4.27 ) explicitly for each $\mathfrak{g}$. In describing the data $\nu^{*}(4.29$ ), we use the symbols:

$$
\begin{aligned}
& \nu=\left\{\nu_{k}\right\}_{k \geq 1}, \quad s \nu=\left\{s \nu_{k}\right\}_{k \geq 1} \quad(s=2,3), \\
& \xi_{2}=\left\{\nu_{\frac{k}{2}}\right\}_{k \geq 1}, \quad \xi_{3}=\left\{\nu_{\frac{k}{3}}\right\}_{k \geq 1} \quad\left(\nu_{\text {non-integer }}=0\right), \\
& \eta_{2}=\left\{2 \nu_{2 k}+\nu_{2 k-1}+\nu_{2 k+1}\right\}_{k \geq 1}, \\
& \eta_{3}=\left\{3 \nu_{3 k}+2\left(\nu_{3 k-1}+\nu_{3 k+1}\right)+\nu_{3 k-2}+\nu_{3 k+2}\right\}_{k \geq 1} .
\end{aligned}
$$

To simplify the presentation we find it convenient to introduce the fermionic

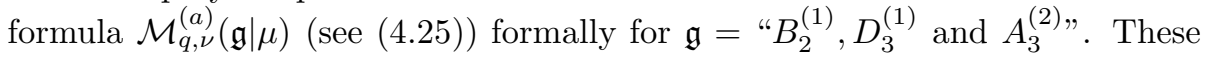
algebras were out of the list in the beginning of Section 2.1, but it is natural to set

$$
\begin{aligned}
& \mathcal{M}_{q, \nu}^{(a)}\left(B_{2}^{(1)} \mid \sum_{c=1}^{2} \mu_{c} \alpha_{c}\right)=\mathcal{M}_{q, \nu}^{(3-a)}\left(C_{2}^{(1)} \mid \sum_{c=1}^{2} \mu_{3-c} \alpha_{c}\right) \quad a=1,2, \\
& \mathcal{M}_{q, \nu}^{(a)}\left(D_{3}^{(1)} \mid \sum_{c=1}^{3} \mu_{c} \alpha_{c}\right)=\mathcal{M}_{q, \nu}^{\left(a^{\prime}\right)}\left(A_{3}^{(1)} \mid \sum_{c=1}^{3} \mu_{c^{\prime}} \alpha_{c}\right) \quad 1 \leq a \leq 3,\left(1^{\prime}, 2^{\prime}, 3^{\prime}\right)=(2,1,3), \\
& \mathcal{M}_{q, \nu}^{(a)}\left(A_{3}^{(2)} \mid \sum_{c=1}^{2} \mu_{c} \alpha_{c}\right)=\mathcal{M}_{q, \nu}^{(3-a)}\left(D_{3}^{(2)} \mid \sum_{c=1}^{2} \mu_{3-c} \alpha_{c}\right) \quad a=1,2 .
\end{aligned}
$$

\footnotetext{
${ }^{19}$ Unlike $\lceil x\rceil$ in Conjecture $2.1(1)$, the symbol $[x]$ here denotes the greatest integer not exceeding $x$.
} 
These relations are relevant to $(\mathfrak{g}, b)=\left(B_{n}^{(1)}, n-2\right),\left(D_{n}^{(1)}, n-3\right)$ and $\left(A_{2 n-1}^{(2)}, n-\right.$ 2 ) in the following. In view of (4.21), there is a symmetry

$$
\mathcal{M}_{q, \nu}^{(a)}(\mathfrak{g} \mid \mu)=\mathcal{M}_{q, \nu}^{(\sigma(a))}(\mathfrak{g} \mid \sigma(\mu)),
$$

for the algebras $\mathfrak{g}=A_{n}^{(1)}, D_{n}^{(1)}$ and $E_{6}^{(2)}$. Therefore the factor $\mathcal{M}_{q, \nu}^{(2)}\left(A_{2}^{(1)} \mid \mu_{1} \alpha_{1}+\right.$ $\left.\mu_{2} \alpha_{2}\right)$ for example in $(\mathfrak{g}, b)=\left(E_{6}^{(1)}, 3\right)$ can also be written as $\mathcal{M}_{q, \nu}^{(1)}\left(A_{2}^{(1)} \mid \mu_{2} \alpha_{1}+\right.$ $\left.\mu_{1} \alpha_{2}\right)$. There are several places in the following results where one has a freedom to make such replacements. Finally we employ the convention $\mathcal{M}_{q, \nu}^{(a)}\left(A_{0}^{(1)} \mid \mu\right)=1$. $\mathfrak{g}=A_{n}^{(1)}$ :

$$
\sum_{\nu} \frac{q^{\psi}}{\prod_{i}(q)_{\nu_{i}}} \mathcal{M}_{q, \nu}^{(b-1)}\left(A_{b-1}^{(1)} \mid \sum_{j=1}^{b-1} \mu_{j} \alpha_{j}\right) \mathcal{M}_{q, \nu}^{(1)}\left(A_{n-b}^{(1)} \mid \sum_{j=1}^{n-b} \mu_{b+j} \alpha_{j}\right) .
$$

$\mathfrak{g}=B_{n}^{(1)}$ :

$$
\begin{array}{ll}
b \leq n-2, \quad & \sum_{\nu} \frac{q^{\psi}}{\prod_{i}(q)_{\nu_{i}}} \mathcal{M}_{q, \nu}^{(b-1)}\left(A_{b-1}^{(1)} \mid \sum_{j=1}^{b-1} \mu_{j} \alpha_{j}\right) \mathcal{M}_{q, \nu}^{(1)}\left(B_{n-b}^{(1)} \mid \sum_{j=1}^{n-b} \mu_{b+j} \alpha_{j}\right), \\
b=n-1, & \sum_{\nu} \frac{q^{\psi}}{\prod_{i}(q)_{\nu_{i}}} \mathcal{M}_{q, \nu}^{(n-2)}\left(A_{n-2}^{(1)} \mid \sum_{j=1}^{n-2} \mu_{j} \alpha_{j}\right) \mathcal{M}_{q, \xi_{2}}^{(1)}\left(A_{1}^{(1)} \mid \mu_{n} \alpha_{1}\right), \\
b=n, & \sum_{\nu} \frac{q^{\psi}}{\prod_{i}(q)_{\nu_{i}}} \mathcal{M}_{q, \eta_{2}}^{(n-1)}\left(A_{n-1}^{(1)} \mid \sum_{j=1}^{n-1} \mu_{j} \alpha_{j}\right) .
\end{array}
$$

$\mathfrak{g}=C_{n}^{(1)}:$

$$
\begin{array}{ll}
b \leq n-2, & \sum_{\nu} \frac{q^{\psi}}{\prod_{i}(q)_{\nu_{i}}} \mathcal{M}_{q, \nu}^{(b-1)}\left(A_{b-1}^{(1)} \mid \sum_{j=1}^{b-1} \mu_{j} \alpha_{j}\right) \mathcal{M}_{q, \nu}^{(1)}\left(C_{n-b}^{(1)} \mid \sum_{j=1}^{n-b} \mu_{b+j} \alpha_{j}\right), \\
b=n-1, \quad \sum_{\nu} \frac{q^{\psi}}{\prod_{i}(q)_{\nu_{i}}} \mathcal{M}_{q, \nu}^{(n-2)}\left(A_{n-2}^{(1)} \mid \sum_{j=1}^{n-2} \mu_{j} \alpha_{j}\right) \mathcal{M}_{q, \eta_{2}}^{(1)}\left(A_{1}^{(1)} \mid \mu_{n} \alpha_{1}\right), \\
b=n, \quad \sum_{\nu} \frac{q^{\psi}}{\prod_{i}(q)_{\nu_{i}}} \mathcal{M}_{q, \xi_{2}}^{(n-1)}\left(A_{n-1}^{(1)} \mid \sum_{j=1}^{n-1} \mu_{j} \alpha_{j}\right) .
\end{array}
$$

$\mathfrak{g}=D_{n}^{(1)}:$

$$
\begin{aligned}
& b \leq n-3, \quad \sum_{\nu} \frac{q^{\psi}}{\prod_{i}(q)_{\nu_{i}}} \mathcal{M}_{q, \nu}^{(b-1)}\left(A_{b-1}^{(1)} \mid \sum_{j=1}^{b-1} \mu_{j} \alpha_{j}\right) \mathcal{M}_{q, \nu}^{(1)}\left(D_{n-b}^{(1)} \mid \sum_{j=1}^{n-b} \mu_{b+j} \alpha_{j}\right), \\
& b=n-2, \quad \sum_{\nu} \frac{q^{\psi}}{\prod_{i}(q)_{\nu_{i}}} \mathcal{M}_{q, \nu}^{(n-3)}\left(A_{n-3}^{(1)} \mid \sum_{j=1}^{n-3} \mu_{j} \alpha_{j}\right) \mathcal{M}_{q, \nu}^{(1)}\left(A_{1}^{(1)} \mid \mu_{n-1} \alpha_{1}\right) \mathcal{M}_{q, \nu}^{(1)}\left(A_{1}^{(1)} \mid \mu_{n} \alpha_{1}\right), \\
& b=n-1, n, \sum_{\nu} \frac{q^{\psi}}{\prod_{i}(q)_{\nu_{i}}} \mathcal{M}_{q, \nu}^{(n-2)}\left(A_{n-1}^{(1)} \mid \sum_{j=1}^{n-2} \mu_{j} \alpha_{j}+\mu_{\bar{b}} \alpha_{n-1}\right),
\end{aligned}
$$


where in the last line, $\bar{b}$ is determined by $\{b, \bar{b}\}=\{n-1, n\}$.

$\mathfrak{g}=E_{6}^{(1)}:$

$b=1,5, \quad \sum_{\nu} \frac{q^{\psi}}{\prod_{i}(q)_{\nu_{i}}} \mathcal{M}_{q, \nu}^{(4)}\left(D_{5}^{(1)} \mid \sum_{j=1}^{4} \mu_{((b-3) j-3(b-5)) / 2} \alpha_{j}+\mu_{6} \alpha_{5}\right)$,
$b=2,4, \quad \sum_{\nu} \frac{q^{\psi}}{\prod_{i}(q)_{\nu_{i}}} \mathcal{M}_{q, \nu}^{(1)}\left(A_{1}^{(1)} \mid \mu_{2 b-3} \alpha_{1}\right) \mathcal{M}_{q, \nu}^{(3)}\left(A_{4}^{(1)} \mid \sum_{j=1}^{3} \mu_{(b-3) j-3(b-4)} \alpha_{j}+\mu_{6} \alpha_{4}\right)$,

$b=3, \quad \sum_{\nu} \frac{q^{\psi}}{\prod_{i}(q)_{\nu_{i}}} \mathcal{M}_{q, \nu}^{(2)}\left(A_{2}^{(1)} \mid \mu_{1} \alpha_{1}+\mu_{2} \alpha_{2}\right) \mathcal{M}_{q, \nu}^{(1)}\left(A_{2}^{(1)} \mid \mu_{4} \alpha_{1}+\mu_{5} \alpha_{2}\right) \mathcal{M}_{q, \nu}^{(1)}\left(A_{1}^{(1)} \mid \mu_{6} \alpha_{1}\right)$,

$b=6, \quad \sum_{\nu} \frac{q^{\psi}}{\prod_{i}(q)_{\nu_{i}}} \mathcal{M}_{q, \nu}^{(3)}\left(A_{5}^{(1)} \mid \sum_{j=1}^{5} \mu_{j} \alpha_{j}\right)$.

$\mathfrak{g}=E_{7}^{(1)}:$

$b=1, \quad \sum_{\nu} \frac{q^{\psi}}{\prod_{i}(q)_{\nu_{i}}} \mathcal{M}_{q, \nu}^{(5)}\left(D_{6}^{(1)} \mid \sum_{j=1}^{5} \mu_{7-j} \alpha_{j}+\mu_{7} \alpha_{6}\right)$,

$b=2, \quad \sum_{\nu} \frac{q^{\psi}}{\prod_{i}(q)_{\nu_{i}}} \mathcal{M}_{q, \nu}^{(1)}\left(A_{1}^{(1)} \mid \mu_{1} \alpha_{1}\right) \mathcal{M}_{q, \nu}^{(2)}\left(A_{5}^{(1)} \mid \mu_{7} \alpha_{1}+\sum_{j=2}^{5} \mu_{j+1} \alpha_{j}\right)$,

$b=3, \quad \sum_{\nu} \frac{q^{\psi}}{\prod_{i}(q)_{\nu_{i}}} \mathcal{M}_{q, \nu}^{(2)}\left(A_{2}^{(1)} \mid \mu_{1} \alpha_{1}+\mu_{2} \alpha_{2}\right) \mathcal{M}_{q, \nu}^{(1)}\left(A_{3}^{(1)} \mid \sum_{j=1}^{3} \mu_{j+3} \alpha_{j}\right) \mathcal{M}_{q, \nu}^{(1)}\left(A_{1}^{(1)} \mid \mu_{7} \alpha_{1}\right)$,

$b=4, \quad \sum_{\nu} \frac{q^{\psi}}{\prod_{i}(q)_{\nu_{i}}} \mathcal{M}_{q, \nu}^{(3)}\left(A_{4}^{(1)} \mid \sum_{j=1}^{3} \mu_{j} \alpha_{j}+\mu_{7} \alpha_{4}\right) \mathcal{M}_{q, \nu}^{(1)}\left(A_{2}^{(1)} \mid \mu_{5} \alpha_{1}+\mu_{6} \alpha_{2}\right)$,

$b=5, \quad \sum_{\nu} \frac{q^{\psi}}{\prod_{i}(q)_{\nu_{i}}} \mathcal{M}_{q, \nu}^{(4)}\left(D_{5}^{(1)} \mid \sum_{j=1}^{4} \mu_{j} \alpha_{j}+\mu_{7} \alpha_{5}\right) \mathcal{M}_{q, \nu}^{(1)}\left(A_{1}^{(1)} \mid \mu_{6} \alpha_{1}\right)$,

$b=6, \quad \sum_{\nu} \frac{q^{\psi}}{\prod_{i}(q)_{\nu_{i}}} \mathcal{M}_{q, \nu}^{(5)}\left(E_{6}^{(1)} \mid \sum_{j=1}^{5} \mu_{j} \alpha_{j}+\mu_{7} \alpha_{6}\right)$,

$b=7, \quad \sum_{\nu} \frac{q^{\psi}}{\prod_{i}(q)_{\nu_{i}}} \mathcal{M}_{q, \nu}^{(3)}\left(A_{6}^{(1)} \mid \sum_{j=1}^{6} \mu_{j} \alpha_{j}\right)$. 


$$
\begin{aligned}
& \mathfrak{g}=E_{8}^{(1)}: \\
& b=1, \quad \sum_{\nu} \frac{q^{\psi}}{\prod_{i}(q)_{\nu_{i}}} \mathcal{M}_{q, \nu}^{(6)}\left(E_{7}^{(1)} \mid \sum_{j=1}^{6} \mu_{8-j} \alpha_{j}+\mu_{8} \alpha_{7}\right), \\
& b=2, \quad \sum_{\nu} \frac{q^{\psi}}{\prod_{i}(q)_{\nu_{i}}} \mathcal{M}_{q, \nu}^{(1)}\left(A_{1}^{(1)} \mid \mu_{1} \alpha_{1}\right) \mathcal{M}_{q, \nu}^{(1)}\left(E_{6}^{(1)} \mid \sum_{j=1}^{5} \mu_{j+2} \alpha_{j}+\mu_{8} \alpha_{6}\right), \\
& b=3, \quad \sum_{\nu} \frac{q^{\psi}}{\prod_{i}(q)_{\nu_{i}}} \mathcal{M}_{q, \nu}^{(2)}\left(A_{2}^{(1)} \mid \mu_{1} \alpha_{1}+\mu_{2} \alpha_{2}\right) \mathcal{M}_{q, \nu}^{(4)}\left(D_{5}^{(1)} \mid \sum_{j=1}^{4} \mu_{8-j} \alpha_{j}+\mu_{8} \alpha_{5}\right), \\
& b=4, \quad \sum_{\nu} \frac{q^{\psi}}{\prod_{i}(q)_{\nu_{i}}} \mathcal{M}_{q, \nu}^{(3)}\left(A_{3}^{(1)} \mid \sum_{j=1}^{3} \mu_{j} \alpha_{j}\right) \mathcal{M}_{q, \nu}^{(2)}\left(A_{4}^{(1)} \mid \mu_{8} \alpha_{1}+\sum_{j=2}^{4} \mu_{j+3} \alpha_{j}\right), \\
& b=5, \quad \sum_{\nu} \frac{q^{\psi}}{\prod_{i}(q)_{\nu_{i}}} \mathcal{M}_{q, \nu}^{(4)}\left(A_{4}^{(1)} \mid \sum_{j=1}^{4} \mu_{j} \alpha_{j}\right) \mathcal{M}_{q, \nu}^{(1)}\left(A_{1}^{(1)} \mid \mu_{8} \alpha_{1}\right) \mathcal{M}_{q, \nu}^{(1)}\left(A_{2}^{(1)} \mid \mu_{6} \alpha_{1}+\mu_{7} \alpha_{2}\right), \\
& b=6, \quad \sum_{\nu} \frac{q^{\psi}}{\prod_{i}(q)_{\nu_{i}}} \mathcal{M}_{q, \nu}^{(5)}\left(A_{6}^{(1)} \mid \sum_{j=1}^{5} \mu_{j} \alpha_{j}+\mu_{8} \alpha_{6}\right) \mathcal{M}_{q, \nu}^{(1)}\left(A_{1}^{(1)} \mid \mu_{7} \alpha_{1}\right), \\
& b=7, \quad \sum_{\nu} \frac{q^{\psi}}{\prod_{i}(q)_{\nu_{i}}} \mathcal{M}_{q, \nu}^{(6)}\left(D_{7}^{(1)} \mid \sum_{j=1}^{6} \mu_{j} \alpha_{j}+\mu_{8} \alpha_{7}\right), \\
& b=8, \quad \sum_{\nu} \frac{q^{\psi}}{\prod_{i}(q)_{\nu_{i}}} \mathcal{M}_{q, \nu}^{(5)}\left(A_{7}^{(1)} \mid \sum_{j=1}^{7} \mu_{j} \alpha_{j}\right) . \\
& \mathfrak{g}=F_{4}^{(1)}: \\
& b=1, \quad \sum_{\nu} \frac{q^{\psi}}{\prod_{i}(q)_{\nu_{i}}} \mathcal{M}_{q, \nu}^{(3)}\left(C_{3}^{(1)} \mid \sum_{j=1}^{3} \mu_{5-j} \alpha_{j}\right), \\
& b=2, \quad \sum_{\nu} \frac{q^{\psi}}{\prod_{i}(q)_{\nu_{i}}} \mathcal{M}_{q, \nu}^{(1)}\left(A_{1}^{(1)} \mid \mu_{1} \alpha_{1}\right) \mathcal{M}_{q, \xi_{2}}^{(1)}\left(A_{2}^{(1)} \mid \mu_{3} \alpha_{1}+\mu_{4} \alpha_{2}\right), \\
& b=3, \quad \sum_{\nu} \frac{q^{\psi}}{\prod_{i}(q)_{\nu_{i}}} \mathcal{M}_{q, \eta_{2}}^{(2)}\left(A_{2}^{(1)} \mid \mu_{1} \alpha_{1}+\mu_{2} \alpha_{2}\right) \mathcal{M}_{q, \nu}^{(1)}\left(A_{1}^{(1)} \mid \mu_{4} \alpha_{1}\right), \\
& b=4, \quad \sum_{\nu} \frac{q^{\psi}}{\prod_{i}(q)_{\nu_{i}}} \mathcal{M}_{q, \nu}^{(3)}\left(B_{3}^{(1)} \mid \sum_{j=1}^{3} \mu_{j} \alpha_{j}\right) . \\
& \mathfrak{g}=G_{2}^{(1)}: \\
& b=1, \quad \sum_{\nu} \frac{q^{\psi}}{\prod_{i}(q)_{\nu_{i}}} \mathcal{M}_{q, \xi_{3}}^{(1)}\left(A_{1}^{(1)} \mid \mu_{2} \alpha_{1}\right), \\
& b=2, \quad \sum_{\nu} \frac{q^{\psi}}{\prod_{i}(q)_{\nu_{i}}} \mathcal{M}_{q, \eta_{3}}^{(1)}\left(A_{1}^{(1)} \mid \mu_{1} \alpha_{1}\right) .
\end{aligned}
$$




$$
\begin{aligned}
& \mathfrak{g}=A_{2 n}^{(2)} \\
& b \leq n-1, \quad \sum_{\nu} \frac{q^{2 \psi}}{\prod_{i}\left(q^{2}\right)_{\nu_{i}}} \mathcal{M}_{q^{2}, \nu}^{(b-1)}\left(A_{b-1}^{(1)} \mid \sum_{j=1}^{b-1} \mu_{j} \alpha_{j}\right) \mathcal{M}_{q, \nu}^{(1)}\left(A_{2 n-2 b}^{(2)} \mid \sum_{j=1}^{n-b} \mu_{b+j} \alpha_{j}\right), \\
& b=n, \quad \sum_{\nu} \frac{q^{\psi}}{\prod_{i}\left(q^{2}\right)_{\nu_{i}}} \mathcal{M}_{q^{2}, \nu}^{(n-1)}\left(A_{n-1}^{(1)} \mid \sum_{j=1}^{n-1} \mu_{j} \alpha_{j}\right) . \\
& \mathfrak{g}=A_{2 n-1}^{(2)}: \\
& b \leq n-2, \quad \sum_{\nu} \frac{q^{\psi}}{\prod_{i}(q)_{\nu_{i}}} \mathcal{M}_{q, \nu}^{(b-1)}\left(A_{b-1}^{(1)} \mid \sum_{j=1}^{b-1} \mu_{j} \alpha_{j}\right) \mathcal{M}_{q, \nu}^{(1)}\left(A_{2 n-2 b-1}^{(2)} \mid \sum_{j=1}^{n-b} \mu_{b+j} \alpha_{j}\right), \\
& b=n-1, \quad \sum_{\nu} \frac{q^{\psi}}{\prod_{i}(q)_{\nu_{i}}} \mathcal{M}_{q, \nu}^{(n-2)}\left(A_{n-2}^{(1)} \mid \sum_{j=1}^{n-2} \mu_{j} \alpha_{j}\right) \mathcal{M}_{q^{2}, \nu}^{(1)}\left(A_{1}^{(1)} \mid \mu_{n} \alpha_{1}\right), \\
& b=n, \quad \sum_{\nu} \frac{q^{2 \psi}}{\prod_{i}\left(q^{2}\right)_{\nu_{i}}} \mathcal{M}_{q, 2 \nu}^{(n-1)}\left(A_{n-1}^{(1)} \mid \sum_{j=1}^{n-1} \mu_{j} \alpha_{j}\right) . \\
& \mathfrak{g}=D_{n+1}^{(2)}: \\
& b \leq n-2, \quad \sum_{\nu} \frac{q^{2 \psi}}{\prod_{i}\left(q^{2}\right)_{\nu_{i}}} \mathcal{M}_{q^{2}, \nu}^{(b-1)}\left(A_{b-1}^{(1)} \mid \sum_{j=1}^{b-1} \mu_{j} \alpha_{j}\right) \mathcal{M}_{q, \nu}^{(1)}\left(D_{n-b+1}^{(2)} \mid \sum_{j=1}^{n-b} \mu_{b+j} \alpha_{j}\right), \\
& b=n-1, \quad \sum_{\nu} \frac{q^{2 \psi}}{\prod_{i}\left(q^{2}\right)_{\nu_{i}}} \mathcal{M}_{q^{2}, \nu}^{(n-2)}\left(A_{n-2}^{(1)} \mid \sum_{j=1}^{n-2} \mu_{j} \alpha_{j}\right) \mathcal{M}_{q, 2 \nu}^{(1)}\left(A_{1}^{(1)} \mid \mu_{n} \alpha_{1}\right), \\
& b=n, \quad \sum_{\nu} \frac{q^{\psi}}{\prod_{i}(q)_{\nu_{i}}} \mathcal{M}_{q^{2}, \nu}^{(n-1)}\left(A_{n-1}^{(1)} \mid \sum_{j=1}^{n-1} \mu_{j} \alpha_{j}\right) . \\
& \mathfrak{g}=E_{6}^{(2)}: \\
& b=1, \quad \sum_{\nu} \frac{q^{\psi}}{\prod_{i}(q)_{\nu_{i}}} \mathcal{M}_{q, \nu}^{(3)}\left(D_{4}^{(2)} \mid \sum_{j=1}^{3} \mu_{5-j} \alpha_{j}\right), \\
& b=2, \quad \sum_{\nu} \frac{q^{\psi}}{\prod_{i}(q)_{\nu_{i}}} \mathcal{M}_{q, \nu}^{(1)}\left(A_{1}^{(1)} \mid \mu_{1} \alpha_{1}\right) \mathcal{M}_{q^{2}, \nu}^{(1)}\left(A_{2}^{(1)} \mid \mu_{3} \alpha_{1}+\mu_{4} \alpha_{2}\right), \\
& b=3, \quad \sum_{\nu} \frac{q^{2 \psi}}{\prod_{i}\left(q^{2}\right)_{\nu_{i}}} \mathcal{M}_{q, 2 \nu}^{(2)}\left(A_{2}^{(1)} \mid \mu_{1} \alpha_{1}+\mu_{2} \alpha_{2}\right) \mathcal{M}_{q^{2}, \nu}^{(1)}\left(A_{1}^{(1)} \mid \mu_{4} \alpha_{1}\right), \\
& b=4, \quad \sum_{\nu} \frac{q^{2 \psi}}{\prod_{i}\left(q^{2}\right)_{\nu_{i}}} \mathcal{M}_{q, \nu}^{(3)}\left(A_{5}^{(2)} \mid \sum_{j=1}^{3} \mu_{j} \alpha_{j}\right) .
\end{aligned}
$$


$\mathfrak{g}=D_{4}^{(3)}:$

$$
\begin{aligned}
& b=1, \quad \sum_{\nu} \frac{q^{\psi}}{\prod_{i}(q)_{\nu_{i}}} \mathcal{M}_{q^{3}, \nu}^{(1)}\left(A_{1}^{(1)} \mid \mu_{2} \alpha_{1}\right), \\
& b=2, \quad \sum_{\nu} \frac{q^{3 \psi}}{\prod_{i}\left(q^{3}\right)_{\nu_{i}}} \mathcal{M}_{q, 3 \nu}^{(1)}\left(A_{1}^{(1)} \mid \mu_{1} \alpha_{1}\right) .
\end{aligned}
$$

The decomposition into subalgebras for nontwisted cases exhibits the same pattern as the Bethe ansatz calculation of central charges in "regime II" RSOS models. See eq.(3.9) in [Ku].

\section{Solution to $B_{n}^{(1)} Q$-system by $D_{n}$ characters}

Here we present a solution of the unrestricted $Q$-system (6.1) for $\mathfrak{g}=B_{n}^{(1)}$ in terms of the character of a classical subalgebra $\mathfrak{g}^{\prime}=D_{n} \subset B_{n}^{(1)}$. If $\mathfrak{g}$ is nontwisted and non-exceptional, i.e., if $\mathfrak{g}=X_{n}^{(1)}=A_{n}^{(1)}, B_{n}^{(1)}, C_{n}^{(1)}, D_{n}^{(1)}$, this is the unique case where the classical subalgebra $\mathfrak{g}^{\prime} \subset \mathfrak{g}$ obtained by removing an end point of the Dynkin diagram differs from $X_{n}$. A result for $\left(\mathfrak{g}, \mathfrak{g}^{\prime}\right)=$ $\left(B_{n}^{(1)}, B_{n}\right)$ case is available in [KR2, HKOTY1]. Let us introduce the linear combination of characters:

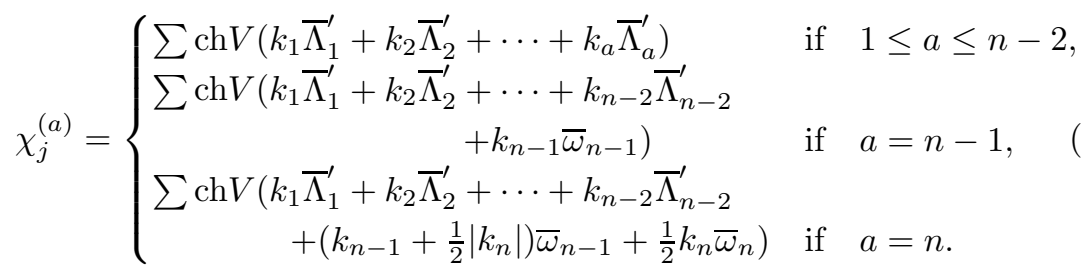

Here $\operatorname{ch} V(\lambda)$ denotes the irreducible $D_{n}$ character with highest weight $\lambda . \bar{\Lambda}_{a}^{\prime}(1 \leq$ $a \leq n)$ are the fundamental weights of $D_{n}$. We have also used the convention $\bar{\Lambda}_{0}^{\prime}=0$ and $\bar{\omega}_{n-1}=\bar{\Lambda}_{n-1}^{\prime}+\bar{\Lambda}_{n}^{\prime}, \bar{\omega}_{n}=-\bar{\Lambda}_{n-1}^{\prime}+\bar{\Lambda}_{n}^{\prime}$. The sums in (C.1) are taken as follows.

$$
\begin{aligned}
& \text { For } \quad a \leq n-1: k_{1}, \ldots, k_{a} \in \mathbb{Z}_{\geq 0}, k_{1} \cdots+k_{a} \leq j, \\
& \text { For } \quad a=n: k_{1}, \ldots, k_{n-1} \in \mathbb{Z}_{\geq 0}, k_{n} \in \mathbb{Z}, 2\left(k_{1}+\cdots+k_{n-1}\right)+\left|k_{n}\right| \leq j, \\
& k_{n}-j \in 2 \mathbb{Z} .
\end{aligned}
$$

If one depicts the highest weights in $(\mathrm{C} .1)_{a \leq n-2}$ with Young diagrams as usual, they correspond to those obtained from the $a \times j$ rectangle by successively removing $1 \times 1$ pieces. We note that $\chi_{j}^{(n-1)}=\operatorname{ch} V\left(j \bar{\Lambda}_{n-1}^{\prime}+j \bar{\Lambda}_{n}^{\prime}\right)+\cdots$ and $\chi_{j}^{(n)}=\operatorname{ch} V\left(j \bar{\Lambda}_{n-1}^{\prime}\right)+\operatorname{ch} V\left(j \bar{\Lambda}_{n-1}^{\prime}\right)+\cdots$.

The $D_{n}$ character $\operatorname{ch} V(\lambda)$ belongs to $\mathbb{Z}\left[x_{1}^{ \pm 1}, \ldots, x_{n}^{ \pm 1}\right]$, where $x_{a}=e^{\bar{\Lambda}_{a}^{\prime}}$ is a complex variable. One can show Theorem 6.2 (B) for $\left(\mathfrak{g}, \mathfrak{g}^{\prime}\right)=\left(B_{n}^{(1)}, D_{n}\right)$ for 
the $\chi_{j}^{(a)}$ given by (C.1). However, (A) and (C) no longer hold. For example instead of $(\mathrm{C})$, one has

$$
\lim _{j \rightarrow \infty}\left(\frac{\chi_{j}^{(a)}}{\chi_{j+1}^{(a)}}\right)= \begin{cases}x_{a}^{-1} & \text { if } 1 \leq a \leq n-2, \\ \left(x_{n-1} x_{n}\right)^{-1} & \text { if } a=n-1, \\ x_{n-1}^{-1} & \text { if } a=n \text { and }\left|e^{\alpha_{n-1}^{\prime}}\right|>\left|e^{\alpha_{n}^{\prime}}\right| \\ x_{n}^{-1} & \text { if } a=n \text { and }\left|e^{\alpha_{n-1}^{\prime}}\right|<\left|e^{\alpha_{n}^{\prime}}\right|\end{cases}
$$

in the domain $\left|e^{\alpha_{1}^{\prime}}\right|, \ldots,\left|e^{\alpha_{n}^{\prime}}\right|>1$, where $\alpha_{a}^{\prime}$ denotes a simple root of $D_{n}$.

The fact that the $D_{n}$ character $\chi_{j}^{(a)}$ satisfies the $Q$-system of type $\mathfrak{g}=B_{n}^{(1)}$ can be shown by using Theorem 7.1 (B) for $B_{n}^{(1)}$ in HKOTY1, a branching rule for $O(2 n+1) \downarrow O(2 n)$ (see for example, [M]) and $O(n) \downarrow S O(n)$ (see for example, p.471 of [KT]).

\section{Examples of combinatorial $\boldsymbol{R}$ matrices}

In this Appendix we provide explicit data of the isomorphism $B^{1, s_{1}} \otimes B^{1, s_{2}} \simeq$ $B^{1, s_{2}} \otimes B^{1, s_{1}}\left(1 \leq s_{2} \leq s_{1} \leq 2\right)$ and energy function for $\mathfrak{g}=B_{3}^{(1)}, C_{2}^{(1)}, D_{4}^{(1)}, A_{3}^{(2)}$, $A_{4}^{(2)}, D_{3}^{(2)}$ and $D_{4}^{(3)}$. Suppose $b \otimes c \simeq \tilde{c} \otimes \tilde{b}$ and $H(b \otimes c)=h$ under the isomorphism $B^{1, s_{1}} \otimes B^{1, s_{2}} \simeq B^{1, s_{2}} \otimes B^{1, s_{1}}$. For each $B^{1, s_{1}} \otimes B^{1, s_{2}}$, we list the data in a table whose row is labeled with $b$ and column with $c$. At the position corresponding to the $b$-th row and $c$-th column, we put $\tilde{c} \cdot \tilde{b}_{h}$ if $s_{1} \neq s_{2}$. If $s_{1}=s_{2}$, the isomorphism is trivial in that $b=\tilde{c}$ and $c=\tilde{b}$, therefore we just put $h$. Due to the limitation of space, tables for $B^{1,2} \otimes B^{1,2}$ for $B_{3}^{(1)}, D_{4}^{(1)}$ and $D_{4}^{(3)}$ are not included.

Let us comment on the relevant earlier results. For $\mathfrak{g}=A_{n}^{(1)}$, there is a simple algorithm to find these data in NY1]. For $\mathfrak{g}$ non-exceptional type other than $A_{n}^{(1)}$, an algorithm is available for general $s_{1}, s_{2}$ based on an insertion scheme [HKOT1, HKOT2]. If $\mathfrak{g}$ is non-exceptional and $s_{1}=s_{2}$, there is a piecewise linear formula to find $H(b \otimes c)$ for general $s_{1}$ in $\mathrm{KKM}$. (Although for $C_{n}^{(1)}$ [KKM only treats $s_{1}$ even case, the results therein are valid also for odd $s_{1}$ under an appropriate adjustment.)

In [KKM], elements of crystals $B^{1, s}$ are labeled by the vectors with nonnegative integer coordinates $\left(x_{1}, \ldots, x_{n}, \bar{x}_{n}, \ldots, \bar{x}_{1}\right)\left(\mathfrak{g}=C_{n}^{(1)}, D_{n}^{(1)}, A_{2 n}^{(2)}, A_{2 n-1}^{(2)}\right)$ or $\left(x_{1}, \ldots, x_{n}, x_{0}, \bar{x}_{n}, \ldots, \bar{x}_{1}\right)\left(\mathfrak{g}=B_{n}^{(1)}, D_{n+1}^{(1)}\right)$. Here we represent them as $1^{x_{1}} \ldots n^{x_{n}}\left(0^{x_{0}}\right) \bar{n}^{\bar{x}_{n}} \ldots \overline{1}^{\bar{x}_{1}}$ if not all of the coordinates are zero. In case they are all zero, such a crystal element is denoted by $\phi$. All the tables given below are typeset by automatically transforming the output from a computer. 
Table 3: $B^{1,1} \otimes B^{1,1}$ for $B_{3}^{(1)}$

\begin{tabular}{l|lllllll}
\hline \multirow{2}{*}{$b$} & & & & $c$ & & & \\
& 0 & 1 & 2 & 3 & $\overline{3}$ & $\overline{2}$ & $\overline{1}$ \\
\hline 0 & 1 & 2 & 2 & 2 & 1 & 1 & 1 \\
1 & 1 & 2 & 1 & 1 & 1 & 1 & 0 \\
2 & 1 & 2 & 2 & 1 & 1 & 1 & 1 \\
3 & 1 & 2 & 2 & 2 & 1 & 1 & 1 \\
$\overline{3}$ & 2 & 2 & 2 & 2 & 2 & 1 & 1 \\
$\overline{2}$ & 2 & 2 & 2 & 2 & 2 & 2 & 1 \\
$\overline{1}$ & 2 & 2 & 2 & 2 & 2 & 2 & 2 \\
\hline
\end{tabular}

Table 4: $B^{1,1} \otimes B^{1,1}$ for $D_{4}^{(1)}$

\begin{tabular}{c|cccccccc}
\hline \multirow{2}{*}{$b$} & \multicolumn{1}{|c}{$c$} & \multicolumn{1}{c}{} \\
& 1 & 2 & 3 & 4 & $\overline{4}$ & $\overline{3}$ & $\overline{2}$ & $\overline{1}$ \\
\hline 1 & 2 & 1 & 1 & 1 & 1 & 1 & 1 & 0 \\
2 & 2 & 2 & 1 & 1 & 1 & 1 & 1 & 1 \\
3 & 2 & 2 & 2 & 1 & 1 & 1 & 1 & 1 \\
4 & 2 & 2 & 2 & 2 & 1 & 1 & 1 & 1 \\
$\overline{4}$ & 2 & 2 & 2 & 1 & 2 & 1 & 1 & 1 \\
$\overline{3}$ & 2 & 2 & 2 & 2 & 2 & 2 & 1 & 1 \\
$\overline{2}$ & 2 & 2 & 2 & 2 & 2 & 2 & 2 & 1 \\
$\overline{1}$ & 2 & 2 & 2 & 2 & 2 & 2 & 2 & 2 \\
\hline
\end{tabular}


Table 5: $B^{1,2} \otimes B^{1,1}$ for $B_{3}^{(1)}$

\begin{tabular}{|c|c|c|c|c|c|c|c|}
\hline \multirow[b]{2}{*}{$b$} & \multicolumn{7}{|c|}{$c$} \\
\hline & 0 & 1 & 2 & 3 & $\overline{3}$ & $\overline{2}$ & $\overline{1}$ \\
\hline 10 & $\overline{0 \cdot 10_{1}}$ & $0 \cdot 11_{2}$ & $1 \cdot 20_{1}$ & $1 \cdot 30_{1}$ & $0 \cdot 1 \overline{3}_{1}$ & $0 \cdot 1 \overline{2}_{1}$ & $1 \cdot 0 \overline{1}_{0}$ \\
\hline 20 & $0 \cdot 20_{1}$ & $0 \cdot 12_{2}$ & $0 \cdot 22_{2}$ & $2 \cdot 30_{1}$ & $0 \cdot 2 \overline{3}_{1}$ & $0 \cdot 1 \overline{1}_{1}$ & $0 \cdot 2 \overline{1}_{1}$ \\
\hline 30 & $0 \cdot 30_{1}$ & $0 \cdot 13_{2}$ & $0 \cdot 23_{2}$ & $0 \cdot 33_{2}$ & $0 \cdot 2 \overline{2}_{1}$ & $0 \cdot 3 \overline{2}_{1}$ & $0 \cdot 3 \overline{1}_{1}$ \\
\hline $0 \overline{3}$ & $0 \cdot 0 \overline{3}_{1}$ & $\overline{3} \cdot 10_{2}$ & $\overline{3} \cdot 20_{2}$ & $\overline{3} \cdot 30_{2}$ & $0 \cdot \overline{33}_{1}$ & $\overline{3} \cdot 0 \overline{2}_{1}$ & $\overline{3} \cdot 0 \overline{1}_{1}$ \\
\hline $0 \overline{2}$ & $0 \cdot 0 \overline{2}_{1}$ & $\overline{2} \cdot 10_{2}$ & $\overline{2} \cdot 20_{2}$ & $\overline{2} \cdot 30_{2}$ & $0 \cdot \overline{32}_{1}$ & $0 \cdot \overline{22}_{1}$ & $\overline{2} \cdot 0 \overline{1}_{1}$ \\
\hline $0 \overline{1}$ & $0 \cdot 0 \overline{1}_{1}$ & $\overline{1} \cdot 10_{2}$ & $\overline{1} \cdot 20_{2}$ & $\overline{1} \cdot 30_{2}$ & $0 \cdot \overline{31}_{1}$ & $0 \cdot \overline{21}_{1}$ & $0 \cdot \overline{11}_{1}$ \\
\hline 11 & $1 \cdot 10_{1}$ & $1 \cdot 11_{2}$ & $1 \cdot 12_{1}$ & $1 \cdot 13_{1}$ & $1 \cdot 1 \overline{3}_{1}$ & $1 \cdot 1 \overline{2}_{1}$ & $1 \cdot 1 \overline{1}_{0}$ \\
\hline 12 & $2 \cdot 10_{1}$ & $2 \cdot 11_{2}$ & $1 \cdot 22_{1}$ & $2 \cdot 13_{1}$ & $2 \cdot 1 \overline{3}_{1}$ & $2 \cdot 1 \overline{2}_{1}$ & $1 \cdot 2 \overline{1}_{0}$ \\
\hline 13 & $3 \cdot 10_{1}$ & $3 \cdot 11_{2}$ & $1 \cdot 23_{1}$ & $1 \cdot 33_{1}$ & $3 \cdot 1 \overline{3}_{1}$ & $3 \cdot 1 \overline{2}_{1}$ & $1 \cdot 3 \overline{1}_{0}$ \\
\hline $1 \overline{3}$ & $1 \cdot 0 \overline{3}_{1}$ & $\overline{3} \cdot 11_{2}$ & $1 \cdot 2 \overline{3}_{1}$ & $1 \cdot 3 \overline{3}_{1}$ & $1 \cdot \overline{33}_{1}$ & $\overline{3} \cdot 1 \overline{2}_{1}$ & $1 \cdot \overline{31}_{0}$ \\
\hline $1 \overline{2}$ & $1 \cdot 0 \overline{2}_{1}$ & $\overline{2} \cdot 11_{2}$ & $1 \cdot 2 \overline{2}_{1}$ & $1 \cdot 3 \overline{2}_{1}$ & $1 \cdot \overline{32}_{1}$ & $1 \cdot \overline{22}_{1}$ & $1 \cdot \overline{21}_{0}$ \\
\hline $1 \overline{1}$ & $2 \cdot 0 \overline{2}_{1}$ & $\overline{1} \cdot 11_{2}$ & $2 \cdot 2 \overline{2}_{1}$ & $2 \cdot 3 \overline{2}_{1}$ & $2 \cdot \overline{32}_{1}$ & $2 \cdot \overline{22}_{1}$ & $1 \cdot \overline{11}_{0}$ \\
\hline 22 & $2 \cdot 20_{1}$ & $2 \cdot 12_{2}$ & $2 \cdot 22_{2}$ & $2 \cdot 23_{1}$ & $2 \cdot 2 \overline{3}_{1}$ & $2 \cdot 1 \overline{1}_{1}$ & $2 \cdot 2 \overline{1}_{1}$ \\
\hline 23 & $3 \cdot 20_{1}$ & $3 \cdot 12_{2}$ & $3 \cdot 22_{2}$ & $2 \cdot 33_{1}$ & $3 \cdot 2 \overline{3}_{1}$ & $3 \cdot 1 \overline{1}_{1}$ & $3 \cdot 2 \overline{1}_{1}$ \\
\hline $2 \overline{3}$ & $2 \cdot 0 \overline{3}_{1}$ & $\overline{3} \cdot 12_{2}$ & $\overline{3} \cdot 22_{2}$ & $2 \cdot 3 \overline{3}_{1}$ & $2 \cdot \overline{33}_{1}$ & $\overline{3} \cdot 1 \overline{1}_{1}$ & $\overline{3} \cdot 2 \overline{1}_{1}$ \\
\hline $2 \overline{2}$ & $3 \cdot 0 \overline{3}_{1}$ & $\overline{2} \cdot 12_{2}$ & $\overline{2} \cdot 22_{2}$ & $3 \cdot 3 \overline{3}_{1}$ & $3 \cdot \overline{33}_{1}$ & $\overline{2} \cdot 1 \overline{1}_{1}$ & $\overline{2} \cdot 2 \overline{1}_{1}$ \\
\hline $2 \overline{1}$ & $2 \cdot 0 \overline{1}_{1}$ & $\overline{1} \cdot 12_{2}$ & $\overline{1} \cdot 22_{2}$ & $2 \cdot 3 \overline{1}_{1}$ & $2 \cdot \overline{31}_{1}$ & $2 \cdot \overline{21}_{1}$ & $2 \cdot \overline{11}_{1}$ \\
\hline 33 & $3 \cdot 30_{1}$ & $3 \cdot 13_{2}$ & $3 \cdot 23_{2}$ & $3 \cdot 33_{2}$ & $3 \cdot 2 \overline{2}_{1}$ & $3 \cdot 3 \overline{2}_{1}$ & $3 \cdot 3 \overline{1}_{1}$ \\
\hline $3 \overline{3}$ & $0 \cdot 3 \overline{3}_{1}$ & $\overline{3} \cdot 13_{2}$ & $\overline{3} \cdot 23_{2}$ & $\overline{3} \cdot 33_{2}$ & $\overline{3} \cdot 2 \overline{2}_{1}$ & $\overline{3} \cdot 3 \overline{2}_{1}$ & $\overline{3} \cdot 3 \overline{1}_{1}$ \\
\hline $3 \overline{2}$ & $3 \cdot 0 \overline{2}_{1}$ & $\overline{2} \cdot 13_{2}$ & $\overline{2} \cdot 23_{2}$ & $\overline{2} \cdot 33_{2}$ & $3 \cdot \overline{32}_{1}$ & $3 \cdot \overline{22}_{1}$ & $\overline{2} \cdot 3 \overline{1}_{1}$ \\
\hline $3 \overline{1}$ & $3 \cdot 0 \overline{1}_{1}$ & $\overline{1} \cdot 13_{2}$ & $\overline{1} \cdot 23_{2}$ & $\overline{1} \cdot 33_{2}$ & $3 \cdot \overline{31}_{1}$ & $3 \cdot \overline{21}_{1}$ & $3 \cdot \overline{11}_{1}$ \\
\hline$\overline{33}$ & $\overline{3} \cdot 0 \overline{3}_{2}$ & $\overline{3} \cdot 1 \overline{3}_{2}$ & $\overline{3} \cdot 2 \overline{3}_{2}$ & $\overline{3} \cdot 3 \overline{3}_{2}$ & $\overline{3} \cdot \overline{33}_{2}$ & $\overline{3} \cdot \overline{32}_{1}$ & $\overline{3} \cdot \overline{31}_{1}$ \\
\hline$\overline{32}$ & $\overline{2} \cdot 0 \overline{3}_{2}$ & $\overline{2} \cdot 1 \overline{3}_{2}$ & $\overline{2} \cdot 2 \overline{3}_{2}$ & $\overline{2} \cdot 3 \overline{3}_{2}$ & $\overline{2} \cdot \overline{33}_{2}$ & $\overline{3} \cdot \overline{22}_{1}$ & $\overline{2} \cdot \overline{31}_{1}$ \\
\hline$\overline{31}$ & $\overline{1} \cdot 0 \overline{3}_{2}$ & $\overline{1} \cdot 1 \overline{3}_{2}$ & $\overline{1} \cdot 2 \overline{3}_{2}$ & $\overline{1} \cdot 3 \overline{3}_{2}$ & $\overline{1} \cdot \overline{33}_{2}$ & $\overline{3} \cdot \overline{21}_{1}$ & $\overline{3} \cdot \overline{11}_{1}$ \\
\hline$\overline{22}$ & $\overline{2} \cdot 0 \overline{2}_{2}$ & $\overline{2} \cdot 1 \overline{2}_{2}$ & $\overline{2} \cdot 2 \overline{2}_{2}$ & $\overline{2} \cdot 3 \overline{2}_{2}$ & $\overline{2} \cdot \overline{32}_{2}$ & $\overline{2} \cdot \overline{22}_{2}$ & $\overline{2} \cdot \overline{21}_{1}$ \\
\hline$\overline{21}$ & $\overline{1} \cdot 0 \overline{2}_{2}$ & $\overline{1} \cdot 1 \overline{2}_{2}$ & $\overline{1} \cdot 2 \overline{2}_{2}$ & $\overline{1} \cdot 3 \overline{2}_{2}$ & $\overline{1} \cdot \overline{32}_{2}$ & $\overline{1} \cdot \overline{22}_{2}$ & $\overline{2} \cdot \overline{11}_{1}$ \\
\hline$\overline{11}$ & $\overline{1} \cdot 0 \overline{1}_{2}$ & $\overline{1} \cdot 1 \overline{1}_{2}$ & $\overline{1} \cdot 2 \overline{1}_{2}$ & $\overline{1} \cdot 3 \overline{1}_{2}$ & $\overline{1} \cdot \overline{31}_{2}$ & $\overline{1} \cdot \overline{21}_{2}$ & $\overline{1} \cdot \overline{11}_{2}$ \\
\hline
\end{tabular}

Table 6: $B^{1,1} \otimes B^{1,1}$ for $C_{2}^{(1)}$

\begin{tabular}{c|cccc}
\hline \multirow{2}{*}{$b$} & \multicolumn{4}{|c}{$c$} \\
& 1 & 2 & $\overline{2}$ & $\overline{1}$ \\
\hline 1 & 1 & 0 & 0 & 0 \\
2 & 1 & 1 & 0 & 0 \\
$\overline{2}$ & 1 & 1 & 1 & 0 \\
$\overline{1}$ & 1 & 1 & 1 & 1 \\
\hline
\end{tabular}


Table 7: $B^{1,2} \otimes B^{1,1}$ for $C_{2}^{(1)}$

\begin{tabular}{c|cccc}
\hline$b$ & \multicolumn{4}{|c}{$c$} \\
& 1 & 2 & $\overline{2}$ & $\overline{1}$ \\
\hline$\phi$ & $1 \cdot 1 \overline{1}_{0}$ & $1 \cdot 2 \overline{1}_{0}$ & $1 \cdot \overline{21}_{0}$ & $1 \cdot \overline{11}_{0}$ \\
11 & $1 \cdot 11_{1}$ & $1 \cdot 12_{0}$ & $1 \cdot 1 \overline{2}_{0}$ & $1 \cdot \phi_{0}$ \\
12 & $2 \cdot 11_{1}$ & $1 \cdot 22_{0}$ & $2 \cdot 1 \overline{2}_{0}$ & $2 \cdot \phi_{0}$ \\
$1 \overline{2}$ & $\overline{2} \cdot 11_{1}$ & $1 \cdot 2 \overline{2}_{0}$ & $1 \cdot \overline{22}_{0}$ & $\overline{2} \cdot \phi_{0}$ \\
$1 \overline{1}$ & $\overline{1} \cdot 11_{1}$ & $2 \cdot 2 \overline{2}_{0}$ & $2 \cdot \overline{22}_{0}$ & $\overline{1} \cdot \phi_{0}$ \\
22 & $2 \cdot 12_{1}$ & $2 \cdot 22_{1}$ & $2 \cdot 1 \overline{1}_{0}$ & $2 \cdot 2 \overline{1}_{0}$ \\
$2 \overline{2}$ & $\overline{2} \cdot 12_{1}$ & $\overline{2} \cdot 22_{1}$ & $\overline{2} \cdot 1 \overline{1}_{0}$ & $\overline{2} \cdot 2 \overline{1}_{0}$ \\
$2 \overline{1}$ & $\overline{1} \cdot 12_{1}$ & $\overline{1} \cdot 22_{1}$ & $2 \cdot \overline{21}_{0}$ & $2 \cdot \overline{11}_{0}$ \\
$\overline{22}$ & $\overline{2} \cdot 1 \overline{2}_{1}$ & $\overline{2} \cdot 2 \overline{2}_{1}$ & $\overline{2} \cdot \overline{22}_{1}$ & $\overline{2} \cdot \overline{21}_{0}$ \\
$\overline{21}$ & $\overline{1} \cdot 1 \overline{2}_{1}$ & $\overline{1} \cdot 2 \overline{2}_{1}$ & $\overline{1} \cdot \overline{22}_{1}$ & $\overline{2} \cdot \overline{11}_{0}$ \\
$\overline{11}$ & $\overline{1} \cdot 1 \overline{1}_{1}$ & $\overline{1} \cdot 2 \overline{1}_{1}$ & $\overline{1} \cdot \overline{21}_{1}$ & $\overline{1} \cdot \overline{11}_{1}$ \\
\hline
\end{tabular}

Table 8: $B^{1,2} \otimes B^{1,2}$ for $C_{2}^{(1)}$

\begin{tabular}{|c|c|c|c|c|c|c|c|c|c|c|c|}
\hline \multirow[b]{2}{*}{$b$} & \multicolumn{11}{|c|}{$c$} \\
\hline & $\phi$ & 11 & 12 & $1 \overline{2}$ & $1 \overline{1}$ & 22 & $2 \overline{2}$ & $2 \overline{1}$ & $\overline{22}$ & $\overline{21}$ & $\overline{11}$ \\
\hline$\phi$ & $\overline{0}$ & 1 & 1 & 1 & 1 & 1 & 1 & 1 & 1 & 1 & 1 \\
\hline 11 & 1 & 2 & 1 & 1 & 0 & 0 & 0 & 0 & 0 & 0 & 0 \\
\hline 12 & 1 & 2 & 1 & 1 & 0 & 1 & 0 & 0 & 0 & 0 & 0 \\
\hline $1 \overline{2}$ & 1 & 2 & 1 & 1 & 0 & 1 & 1 & 0 & 1 & 0 & 0 \\
\hline $1 \overline{1}$ & 1 & 2 & 1 & 1 & 0 & 1 & 1 & 0 & 1 & 0 & 0 \\
\hline 22 & 1 & 2 & 2 & 1 & 1 & 2 & 0 & 1 & 0 & 0 & 0 \\
\hline $2 \overline{2}$ & 1 & 2 & 2 & 1 & 1 & 2 & 0 & 1 & 0 & 0 & 0 \\
\hline $2 \overline{1}$ & 1 & 2 & 2 & 1 & 1 & 2 & 1 & 1 & 1 & 1 & 1 \\
\hline$\overline{22}$ & 1 & 2 & 2 & 2 & 1 & 2 & 2 & 1 & 2 & 1 & 0 \\
\hline$\overline{21}$ & 1 & 2 & 2 & 2 & 1 & 2 & 2 & 1 & 2 & 1 & 1 \\
\hline$\overline{11}$ & 1 & 2 & 2 & 2 & 2 & 2 & 2 & 2 & 2 & 2 & 2 \\
\hline
\end{tabular}


Table 9: $B^{1,2} \otimes B^{1,1}$ for $D_{4}^{(1)}$

\begin{tabular}{|c|c|c|c|c|c|c|c|c|}
\hline \multirow{2}{*}{$b$} & \multicolumn{8}{|c|}{$c$} \\
\hline & 1 & 2 & 3 & 4 & $\overline{4}$ & $\overline{3}$ & $\overline{2}$ & $\overline{1}$ \\
\hline 11 & $1 \cdot 11_{2}$ & $1 \cdot 12_{1}$ & $1 \cdot 13_{1}$ & $1 \cdot 14_{1}$ & $1 \cdot 1 \overline{4}_{1}$ & $1 \cdot 1 \overline{3}_{1}$ & $1 \cdot 1 \overline{2}_{1}$ & $1 \cdot 1 \overline{1}_{0}$ \\
\hline 12 & $2 \cdot 11_{2}$ & $1 \cdot 22_{1}$ & $2 \cdot 13_{1}$ & $2 \cdot 14_{1}$ & $2 \cdot 1 \overline{4}_{1}$ & $2 \cdot 1 \overline{3}_{1}$ & $2 \cdot 1 \overline{2}_{1}$ & $1 \cdot 2 \overline{1}_{0}$ \\
\hline 13 & $3 \cdot 11_{2}$ & $1 \cdot 23_{1}$ & $1 \cdot 33_{1}$ & $3 \cdot 14_{1}$ & $3 \cdot 1 \overline{4}_{1}$ & $3 \cdot 1 \overline{3}_{1}$ & $3 \cdot 1 \overline{2}_{1}$ & $1 \cdot 3 \overline{1}_{0}$ \\
\hline 14 & $4 \cdot 11_{2}$ & $1 \cdot 24_{1}$ & $1 \cdot 34_{1}$ & $1 \cdot 44_{1}$ & $4 \cdot 1 \overline{4}_{1}$ & $4 \cdot 1 \overline{3}_{1}$ & $4 \cdot 1 \overline{2}_{1}$ & $1 \cdot 4 \overline{1}_{0}$ \\
\hline $1 \overline{4}$ & $\overline{4} \cdot 11_{2}$ & $1 \cdot 2 \overline{4}_{1}$ & $1 \cdot 3 \overline{4}_{1}$ & $\overline{4} \cdot 14_{1}$ & $1 \cdot \overline{44}_{1}$ & $\overline{4} \cdot 1 \overline{3}_{1}$ & $\overline{4} \cdot 1 \overline{2}_{1}$ & $1 \cdot \overline{41}_{0}$ \\
\hline $1 \overline{3}$ & $\overline{3} \cdot 11_{2}$ & $1 \cdot 2 \overline{3}_{1}$ & $1 \cdot 3 \overline{3}_{1}$ & $1 \cdot 4 \overline{3}_{1}$ & $1 \cdot \overline{43}_{1}$ & $1 \cdot \overline{33}_{1}$ & $\overline{3} \cdot 1 \overline{2}_{1}$ & $1 \cdot \overline{31}_{0}$ \\
\hline $1 \overline{2}$ & $\overline{2} \cdot 11_{2}$ & $1 \cdot 2 \overline{2}_{1}$ & $1 \cdot 3 \overline{2}_{1}$ & $1 \cdot 4 \overline{2}_{1}$ & $1 \cdot \overline{42}_{1}$ & $1 \cdot \overline{32}_{1}$ & $1 \cdot \overline{22}_{1}$ & $1 \cdot \overline{21}_{0}$ \\
\hline $1 \overline{1}$ & $\overline{1} \cdot 11_{2}$ & $2 \cdot 2 \overline{2}_{1}$ & $2 \cdot 3 \overline{2}_{1}$ & $2 \cdot 4 \overline{2}_{1}$ & $2 \cdot \overline{42}_{1}$ & $2 \cdot \overline{32}_{1}$ & $2 \cdot \overline{22}_{1}$ & $1 \cdot \overline{11}_{0}$ \\
\hline 22 & $2 \cdot 12_{2}$ & $2 \cdot 22_{2}$ & $2 \cdot 23_{1}$ & $2 \cdot 24_{1}$ & $2 \cdot 2 \overline{4}_{1}$ & $2 \cdot 2 \overline{3}_{1}$ & $2 \cdot 1 \overline{1}_{1}$ & $2 \cdot 2 \overline{1}_{1}$ \\
\hline 23 & $3 \cdot 12_{2}$ & $3 \cdot 22_{2}$ & $2 \cdot 33_{1}$ & $3 \cdot 24_{1}$ & $3 \cdot 2 \overline{4}_{1}$ & $3 \cdot 2 \overline{3}_{1}$ & $3 \cdot 1 \overline{1}_{1}$ & $3 \cdot 2 \overline{1}_{1}$ \\
\hline 24 & $4 \cdot 12_{2}$ & $4 \cdot 22_{2}$ & $2 \cdot 34_{1}$ & $2 \cdot 44_{1}$ & $4 \cdot 2 \overline{4}_{1}$ & $4 \cdot 2 \overline{3}_{1}$ & $4 \cdot 1 \overline{1}_{1}$ & $4 \cdot 2 \overline{1}_{1}$ \\
\hline $2 \overline{4}$ & $\overline{4} \cdot 12_{2}$ & $\overline{4} \cdot 22_{2}$ & $2 \cdot 3 \overline{4}_{1}$ & $\overline{4} \cdot 24_{1}$ & $2 \cdot \overline{44}_{1}$ & $\overline{4} \cdot 2 \overline{3}_{1}$ & $\overline{4} \cdot 1 \overline{1}_{1}$ & $\overline{4} \cdot 2 \overline{1}_{1}$ \\
\hline $2 \overline{3}$ & $\overline{3} \cdot 12_{2}$ & $\overline{3} \cdot 22_{2}$ & $2 \cdot 3 \overline{3}_{1}$ & $2 \cdot 4 \overline{3}_{1}$ & $2 \cdot \overline{43}_{1}$ & $2 \cdot \overline{33}_{1}$ & $\overline{3} \cdot 1 \overline{1}_{1}$ & $\overline{3} \cdot 2 \overline{1}_{1}$ \\
\hline $2 \overline{2}$ & $\overline{2} \cdot 12_{2}$ & $\overline{2} \cdot 22_{2}$ & $3 \cdot 3 \overline{3}_{1}$ & $3 \cdot 4 \overline{3}_{1}$ & $3 \cdot \overline{43}_{1}$ & $3 \cdot \overline{33}_{1}$ & $\overline{2} \cdot 1 \overline{1}_{1}$ & $\overline{2} \cdot 2 \overline{1}_{1}$ \\
\hline $2 \overline{1}$ & $\overline{1} \cdot 12_{2}$ & $\overline{1} \cdot 22_{2}$ & $2 \cdot 3 \overline{1}_{1}$ & $2 \cdot 4 \overline{1}_{1}$ & $2 \cdot \overline{41}_{1}$ & $2 \cdot \overline{31}_{1}$ & $2 \cdot \overline{21}_{1}$ & $2 \cdot \overline{11}_{1}$ \\
\hline 33 & $3 \cdot 13_{2}$ & $3 \cdot 23_{2}$ & $3 \cdot 33_{2}$ & $3 \cdot 34_{1}$ & $3 \cdot 3 \overline{4}_{1}$ & $3 \cdot 2 \overline{2}_{1}$ & $3 \cdot 3 \overline{2}_{1}$ & $3 \cdot 3 \overline{1}_{1}$ \\
\hline 34 & $4 \cdot 13_{2}$ & $4 \cdot 23_{2}$ & $4 \cdot 33_{2}$ & $3 \cdot 44_{1}$ & $4 \cdot 3 \overline{4}_{1}$ & $4 \cdot 2 \overline{2}_{1}$ & $4 \cdot 3 \overline{2}_{1}$ & $4 \cdot 3 \overline{1}_{1}$ \\
\hline $3 \overline{4}$ & $\overline{4} \cdot 13_{2}$ & $\overline{4} \cdot 23_{2}$ & $\overline{4} \cdot 33_{2}$ & $\overline{4} \cdot 34_{1}$ & $3 \cdot \overline{44}_{1}$ & $\overline{4} \cdot 2 \overline{2}_{1}$ & $\overline{4} \cdot 3 \overline{2}_{1}$ & $\overline{4} \cdot 3 \overline{1}_{1}$ \\
\hline $3 \overline{3}$ & $\overline{3} \cdot 13_{2}$ & $\overline{3} \cdot 23_{2}$ & $\overline{3} \cdot 33_{2}$ & $\overline{4} \cdot 44_{1}$ & $4 \cdot \overline{44}_{1}$ & $\overline{3} \cdot 2 \overline{2}_{1}$ & $\overline{3} \cdot 3 \overline{2}_{1}$ & $\overline{3} \cdot 3 \overline{1}_{1}$ \\
\hline $3 \overline{2}$ & $\overline{2} \cdot 13_{2}$ & $\overline{2} \cdot 23_{2}$ & $\overline{2} \cdot 33_{2}$ & $3 \cdot 4 \overline{2}_{1}$ & $3 \cdot \overline{42}_{1}$ & $3 \cdot \overline{32}_{1}$ & $3 \cdot \overline{22}_{1}$ & $\overline{2} \cdot 3 \overline{1}_{1}$ \\
\hline $3 \overline{1}$ & $\overline{1} \cdot 13_{2}$ & $\overline{1} \cdot 23_{2}$ & $\overline{1} \cdot 33_{2}$ & $3 \cdot 4 \overline{1}_{1}$ & $3 \cdot \overline{41}_{1}$ & $3 \cdot \overline{31}_{1}$ & $3 \cdot \overline{21}_{1}$ & $3 \cdot \overline{11}_{1}$ \\
\hline 44 & $4 \cdot 14_{2}$ & $4 \cdot 24_{2}$ & $4 \cdot 34_{2}$ & $4 \cdot 44_{2}$ & $4 \cdot 3 \overline{3}_{1}$ & $4 \cdot 4 \overline{3}_{1}$ & $4 \cdot 4 \overline{2}_{1}$ & $4 \cdot 4 \overline{1}_{1}$ \\
\hline $4 \overline{3}$ & $\overline{3} \cdot 14_{2}$ & $\overline{3} \cdot 24_{2}$ & $\overline{3} \cdot 34_{2}$ & $\overline{3} \cdot 44_{2}$ & $4 \cdot \overline{43}_{1}$ & $4 \cdot \overline{33}_{1}$ & $\overline{3} \cdot 4 \overline{2}_{1}$ & $\overline{3} \cdot 4 \overline{1}_{1}$ \\
\hline $4 \overline{2}$ & $\overline{2} \cdot 14_{2}$ & $\overline{2} \cdot 24_{2}$ & $\overline{2} \cdot 34_{2}$ & $\overline{2} \cdot 44_{2}$ & $4 \cdot \overline{42}_{1}$ & $4 \cdot \overline{32}_{1}$ & $4 \cdot \overline{22}_{1}$ & $\overline{2} \cdot 4 \overline{1}_{1}$ \\
\hline $4 \overline{1}$ & $\overline{1} \cdot 14_{2}$ & $\overline{1} \cdot 24_{2}$ & $\overline{1} \cdot 34_{2}$ & $\overline{1} \cdot 44_{2}$ & $4 \cdot \overline{41}_{1}$ & $4 \cdot \overline{31}_{1}$ & $4 \cdot \overline{21}_{1}$ & $4 \cdot \overline{11}_{1}$ \\
\hline$\overline{44}$ & $\overline{4} \cdot 1 \overline{4}_{2}$ & $\overline{4} \cdot 2 \overline{4}_{2}$ & $\overline{4} \cdot 3 \overline{4}_{2}$ & $\overline{4} \cdot 3 \overline{3}_{1}$ & $\overline{4} \cdot \overline{44}_{2}$ & $\overline{4} \cdot \overline{43}_{1}$ & $\overline{4} \cdot \overline{42}_{1}$ & $\overline{4} \cdot \overline{41}_{1}$ \\
\hline$\overline{43}$ & $\overline{3} \cdot 1 \overline{4}_{2}$ & $\overline{3} \cdot 2 \overline{4}_{2}$ & $\overline{3} \cdot 3 \overline{4}_{2}$ & $\overline{4} \cdot 4 \overline{3}_{1}$ & $\overline{3} \cdot \overline{44}_{2}$ & $\overline{4} \cdot \overline{33}_{1}$ & $\overline{3} \cdot \overline{42}_{1}$ & $\overline{3} \cdot \overline{41}_{1}$ \\
\hline$\overline{42}$ & $\overline{2} \cdot 1 \overline{4}_{2}$ & $\overline{2} \cdot 2 \overline{4}_{2}$ & $\overline{2} \cdot 3 \overline{4}_{2}$ & $\overline{4} \cdot 4 \overline{2}_{1}$ & $\overline{2} \cdot \overline{44}_{2}$ & $\overline{4} \cdot \overline{32}_{1}$ & $\overline{4} \cdot \overline{22}_{1}$ & $\overline{2} \cdot \overline{41}_{1}$ \\
\hline$\overline{41}$ & $\overline{1} \cdot 1 \overline{4}_{2}$ & $\overline{1} \cdot 2 \overline{4}_{2}$ & $\overline{1} \cdot 3 \overline{4}_{2}$ & $\overline{4} \cdot 4 \overline{1}_{1}$ & $\overline{1} \cdot \overline{44}_{2}$ & $\overline{4} \cdot \overline{31}_{1}$ & $\overline{4} \cdot \overline{21}_{1}$ & $\overline{4} \cdot \overline{11}_{1}$ \\
\hline$\overline{33}$ & $\overline{3} \cdot 1 \overline{3}_{2}$ & $\overline{3} \cdot 2 \overline{3}_{2}$ & $\overline{3} \cdot 3 \overline{3}_{2}$ & $\overline{3} \cdot 4 \overline{3}_{2}$ & $\overline{3} \cdot \overline{43}_{2}$ & $\overline{3} \cdot \overline{33}_{2}$ & $\overline{3} \cdot \overline{32}_{1}$ & $\overline{3} \cdot \overline{31}_{1}$ \\
\hline$\overline{32}$ & $\overline{2} \cdot 1 \overline{3}_{2}$ & $\overline{2} \cdot 2 \overline{3}_{2}$ & $\overline{2} \cdot 3 \overline{3}_{2}$ & $\overline{2} \cdot 4 \overline{3}_{2}$ & $\overline{2} \cdot \overline{43}_{2}$ & $\overline{2} \cdot \overline{33}_{2}$ & $\overline{3} \cdot \overline{22}_{1}$ & $\overline{2} \cdot \overline{31}_{1}$ \\
\hline$\overline{31}$ & $\overline{1} \cdot 1 \overline{3}_{2}$ & $\overline{1} \cdot 2 \overline{3}_{2}$ & $\overline{1} \cdot 3 \overline{3}_{2}$ & $\overline{1} \cdot 4 \overline{3}_{2}$ & $\overline{1} \cdot \overline{43}_{2}$ & $\overline{1} \cdot \overline{33}_{2}$ & $\overline{3} \cdot \overline{21}_{1}$ & $\overline{3} \cdot \overline{11}_{1}$ \\
\hline$\overline{22}$ & $\overline{2} \cdot 1 \overline{2}_{2}$ & $\overline{2} \cdot 2 \overline{2}_{2}$ & $\overline{2} \cdot 3 \overline{2}_{2}$ & $\overline{2} \cdot 4 \overline{2}_{2}$ & $\overline{2} \cdot \overline{42}_{2}$ & $\overline{2} \cdot \overline{32}_{2}$ & $\overline{2} \cdot \overline{22}_{2}$ & $\overline{2} \cdot \overline{21}_{1}$ \\
\hline$\overline{21}$ & $\overline{1} \cdot 1 \overline{2}_{2}$ & $\overline{1} \cdot 2 \overline{2}_{2}$ & $\overline{1} \cdot 3 \overline{2}_{2}$ & $\overline{1} \cdot 4 \overline{2}_{2}$ & $\overline{1} \cdot \overline{42}_{2}$ & $\overline{1} \cdot \overline{32}_{2}$ & $\overline{1} \cdot \overline{22}_{2}$ & $\overline{2} \cdot \overline{11}_{1}$ \\
\hline$\overline{11}$ & $\overline{1} \cdot 1 \overline{1}_{2}$ & $\overline{1} \cdot 2 \overline{1}_{2}$ & $\overline{1} \cdot 3 \overline{1}_{2}$ & $\overline{1} \cdot 4 \overline{1}_{2}$ & $\overline{1} \cdot \overline{41}_{2}$ & $\overline{1} \cdot \overline{31}_{2}$ & $\overline{1} \cdot \overline{21}_{2}$ & $\overline{1} \cdot \overline{11}_{2}$ \\
\hline
\end{tabular}


Table $10: B^{1,1} \otimes B^{1,1}$ for $A_{3}^{(2)}$

\begin{tabular}{c|cccc}
\hline \multirow{2}{*}{$b$} & \multicolumn{4}{|c}{$c$} \\
& 1 & 2 & $\overline{2}$ & $\overline{1}$ \\
\hline 1 & 2 & 1 & 1 & 0 \\
2 & 2 & 2 & 1 & 1 \\
$\overline{2}$ & 2 & 2 & 2 & 1 \\
$\overline{1}$ & 2 & 2 & 2 & 2 \\
\hline
\end{tabular}

Table 11: $B^{1,2} \otimes B^{1,1}$ for $A_{3}^{(2)}$

\begin{tabular}{c|cccc}
\hline$b$ & \multicolumn{4}{|c}{$c$} \\
& 1 & 2 & $\overline{2}$ & $\overline{1}$ \\
\hline 11 & $1 \cdot 11_{2}$ & $1 \cdot 12_{1}$ & $1 \cdot 1 \overline{2}_{1}$ & $1 \cdot 1 \overline{1}_{0}$ \\
12 & $2 \cdot 11_{2}$ & $1 \cdot 22_{1}$ & $2 \cdot 1 \overline{2}_{1}$ & $1 \cdot 2 \overline{1}_{0}$ \\
$1 \overline{2}$ & $\overline{2} \cdot 11_{2}$ & $1 \cdot 2 \overline{2}_{1}$ & $1 \cdot \overline{22}_{1}$ & $1 \cdot \overline{21}_{0}$ \\
$1 \overline{1}$ & $\overline{1} \cdot 11_{2}$ & $2 \cdot 2 \overline{2}_{1}$ & $2 \cdot \overline{22}_{1}$ & $1 \cdot \overline{11}_{0}$ \\
22 & $2 \cdot 12_{2}$ & $2 \cdot 22_{2}$ & $2 \cdot 1 \overline{1}_{1}$ & $2 \cdot 2 \overline{1}_{1}$ \\
$2 \overline{2}$ & $\overline{2} \cdot 12_{2}$ & $\overline{2} \cdot 22_{2}$ & $\overline{2} \cdot 1 \overline{1}_{1}$ & $\overline{2} \cdot 2 \overline{1}_{1}$ \\
$2 \overline{1}$ & $\overline{1} \cdot 12_{2}$ & $\overline{1} \cdot 22_{2}$ & $2 \cdot \overline{21}_{1}$ & $2 \cdot \overline{11}_{1}$ \\
$\overline{22}$ & $\overline{2} \cdot 1 \overline{2}_{2}$ & $\overline{2} \cdot 2 \overline{2}_{2}$ & $\overline{2} \cdot \overline{22}_{2}$ & $\overline{2} \cdot \overline{21}_{1}$ \\
$\overline{21}$ & $\overline{1} \cdot 1 \overline{2}_{2}$ & $\overline{1} \cdot 2 \overline{2}_{2}$ & $\overline{1} \cdot \overline{22}_{2}$ & $\overline{2} \cdot \overline{11}_{1}$ \\
$\overline{11}$ & $\overline{1} \cdot 1 \overline{1}_{2}$ & $\overline{1} \cdot 2 \overline{1}_{2}$ & $\overline{1} \cdot \overline{21}_{2}$ & $\overline{1} \cdot \overline{11}_{2}$ \\
\hline
\end{tabular}

Table $12: B^{1,2} \otimes B^{1,2}$ for $A_{3}^{(2)}$

\begin{tabular}{c|cccccccccc}
\hline \multirow{2}{*}{$b$} & \multicolumn{1}{|c}{${ }^{1}$} & 12 & $1 \overline{2}$ & $1 \overline{1}$ & 22 & $2 \overline{2}$ & $2 \overline{1}$ & $\overline{22}$ & $\overline{21}$ & $\overline{11}$ \\
\hline 11 & 4 & 3 & 3 & 2 & 2 & 2 & 1 & 2 & 1 & 0 \\
12 & 4 & 3 & 3 & 2 & 3 & 2 & 2 & 2 & 1 & 1 \\
$1 \overline{2}$ & 4 & 3 & 3 & 2 & 3 & 3 & 2 & 3 & 2 & 1 \\
$1 \overline{1}$ & 4 & 3 & 3 & 2 & 3 & 3 & 2 & 3 & 2 & 2 \\
22 & 4 & 4 & 3 & 3 & 4 & 2 & 3 & 2 & 2 & 2 \\
$2 \overline{2}$ & 4 & 4 & 3 & 3 & 4 & 2 & 3 & 2 & 2 & 2 \\
$2 \overline{1}$ & 4 & 4 & 3 & 3 & 4 & 3 & 3 & 3 & 3 & 3 \\
$\overline{22}$ & 4 & 4 & 4 & 3 & 4 & 4 & 3 & 4 & 3 & 2 \\
$\overline{21}$ & 4 & 4 & 4 & 3 & 4 & 4 & 3 & 4 & 3 & 3 \\
$\overline{11}$ & 4 & 4 & 4 & 4 & 4 & 4 & 4 & 4 & 4 & 4 \\
\hline
\end{tabular}


Table 13: $B^{1,1} \otimes B^{1,1}$ for $A_{4}^{(2)}$

\begin{tabular}{c|ccccc}
\hline \multirow{2}{*}{$b$} & & & $c$ & & \\
& $\phi$ & 1 & 2 & $\overline{2}$ & $\overline{1}$ \\
\hline$\phi$ & 0 & 1 & 1 & 1 & 1 \\
1 & 1 & 2 & 0 & 0 & 0 \\
2 & 1 & 2 & 2 & 0 & 0 \\
$\overline{2}$ & 1 & 2 & 2 & 2 & 0 \\
$\overline{1}$ & 1 & 2 & 2 & 2 & 2 \\
\hline
\end{tabular}

Table 14: $B^{1,2} \otimes B^{1,1}$ for $A_{4}^{(2)}$

\begin{tabular}{c|ccccc}
\hline$b$ & & & $c$ & & \\
& $\phi$ & 1 & 2 & $\overline{2}$ & $\overline{1}$ \\
\hline$\phi$ & $\phi \cdot \phi_{0}$ & $1 \cdot 1 \overline{1}_{0}$ & $1 \cdot 2 \overline{1}_{0}$ & $1 \cdot \overline{21}_{0}$ & $1 \cdot \overline{1}_{0}$ \\
1 & $\phi \cdot 1_{0}$ & $\phi \cdot 11_{1}$ & $1 \cdot 2_{0}$ & $1 \cdot \overline{2}_{0}$ & $1 \cdot \overline{1}_{0}$ \\
2 & $\phi \cdot 2_{0}$ & $\phi \cdot 12_{1}$ & $\phi \cdot 22_{1}$ & $2 \cdot \overline{2}_{0}$ & $2 \cdot \overline{1}_{0}$ \\
$\overline{2}$ & $\phi \cdot \overline{2}_{0}$ & $\phi \cdot 1 \overline{2}_{1}$ & $\phi \cdot 2 \overline{2}_{1}$ & $\phi \cdot \overline{22}_{1}$ & $\overline{2} \cdot \overline{1}_{0}$ \\
$\overline{1}$ & $\phi \cdot \overline{1}_{0}$ & $\phi \cdot 1 \overline{1}_{1}$ & $\phi \cdot 2 \overline{1}_{1}$ & $\phi \cdot \overline{21}_{1}$ & $\phi \cdot \overline{11}_{1}$ \\
11 & $1 \cdot 1_{1}$ & $1 \cdot 11_{2}$ & $1 \cdot 12_{0}$ & $1 \cdot 1 \overline{2}_{0}$ & $1 \cdot \phi_{0}$ \\
12 & $2 \cdot 1_{1}$ & $2 \cdot 11_{2}$ & $1 \cdot 22_{0}$ & $2 \cdot 1 \overline{2}_{0}$ & $2 \cdot \phi_{0}$ \\
$1 \overline{2}$ & $\overline{2} \cdot 1_{1}$ & $\overline{2} \cdot 11_{2}$ & $1 \cdot 2 \overline{2}_{0}$ & $1 \cdot \overline{22}_{0}$ & $\overline{2} \cdot \phi_{0}$ \\
$1 \overline{1}$ & $\overline{1} \cdot 1_{1}$ & $\overline{1} \cdot 11_{2}$ & $2 \cdot 2 \overline{2}_{0}$ & $2 \cdot \overline{22}_{0}$ & $\overline{1} \cdot \phi_{0}$ \\
22 & $2 \cdot 2_{1}$ & $2 \cdot 12_{2}$ & $2 \cdot 22_{2}$ & $2 \cdot 1 \overline{1}_{0}$ & $2 \cdot 2 \overline{1}_{0}$ \\
$2 \overline{2}$ & $\overline{2} \cdot 2_{1}$ & $\overline{2} \cdot 12_{2}$ & $\overline{2} \cdot 22_{2}$ & $\overline{2} \cdot 1 \overline{1}_{0}$ & $\overline{2} \cdot 2 \overline{1}_{0}$ \\
$2 \overline{1}$ & $\overline{1} \cdot 2_{1}$ & $\overline{1} \cdot 12_{2}$ & $\overline{1} \cdot 22_{2}$ & $2 \cdot \overline{21}_{0}$ & $2 \cdot \overline{11}_{0}$ \\
$\overline{22}$ & $\overline{2} \cdot \overline{2}_{1}$ & $\overline{2} \cdot 1 \overline{2}_{2}$ & $\overline{2} \cdot 2 \overline{2}_{2}$ & $\overline{2} \cdot \overline{22}_{2}$ & $\overline{2} \cdot \overline{21}_{0}$ \\
$\overline{21}$ & $\overline{1} \cdot \overline{2}_{1}$ & $\overline{1} \cdot 1 \overline{2}_{2}$ & $\overline{1} \cdot 2 \overline{2}_{2}$ & $\overline{1} \cdot \overline{22}_{2}$ & $\overline{2} \cdot \overline{11}_{0}$ \\
$\overline{11}$ & $\overline{1} \cdot \overline{1}_{1}$ & $\overline{1} \cdot 1 \overline{1}_{2}$ & $\overline{1} \cdot 2 \overline{1}_{2}$ & $\overline{1} \cdot \overline{21}_{2}$ & $\overline{1} \cdot \overline{11}_{2}$ \\
\hline
\end{tabular}


Table $15: B^{1,2} \otimes B^{1,2}$ for $A_{4}^{(2)}$

\begin{tabular}{c|ccccccccccccccc}
\hline \multirow{2}{*}{$b$} & $\phi$ & 1 & 2 & $\overline{2}$ & $\overline{1}$ & 11 & 12 & $1 \overline{2}$ & $1 \overline{1}$ & 22 & $2 \overline{2}$ & $2 \overline{1}$ & $\overline{22}$ & $\overline{21}$ & $\overline{11}$ \\
\hline$\phi$ & 0 & 1 & 1 & 1 & 1 & 2 & 2 & 2 & 2 & 2 & 2 & 2 & 2 & 2 & 2 \\
1 & 1 & 2 & 0 & 0 & 0 & 3 & 1 & 1 & 1 & 1 & 1 & 1 & 1 & 1 & 1 \\
2 & 1 & 2 & 2 & 0 & 0 & 3 & 3 & 1 & 1 & 3 & 1 & 1 & 1 & 1 & 1 \\
$\overline{2}$ & 1 & 2 & 2 & 2 & 0 & 3 & 3 & 3 & 1 & 3 & 3 & 1 & 3 & 1 & 1 \\
$\overline{1}$ & 1 & 2 & 2 & 2 & 2 & 3 & 3 & 3 & 3 & 3 & 3 & 3 & 3 & 3 & 3 \\
11 & 2 & 3 & 1 & 1 & 1 & 4 & 2 & 2 & 0 & 0 & 0 & 0 & 0 & 0 & 0 \\
12 & 2 & 3 & 1 & 1 & 1 & 4 & 2 & 2 & 0 & 2 & 0 & 0 & 0 & 0 & 0 \\
$1 \overline{2}$ & 2 & 3 & 1 & 1 & 1 & 4 & 2 & 2 & 0 & 2 & 2 & 0 & 2 & 0 & 0 \\
$1 \overline{1}$ & 2 & 3 & 1 & 1 & 1 & 4 & 2 & 2 & 0 & 2 & 2 & 0 & 2 & 0 & 0 \\
22 & 2 & 3 & 3 & 1 & 1 & 4 & 4 & 2 & 2 & 4 & 0 & 2 & 0 & 0 & 0 \\
$2 \overline{2}$ & 2 & 3 & 3 & 1 & 1 & 4 & 4 & 2 & 2 & 4 & 0 & 2 & 0 & 0 & 0 \\
$2 \overline{1}$ & 2 & 3 & 3 & 1 & 1 & 4 & 4 & 2 & 2 & 4 & 2 & 2 & 2 & 2 & 2 \\
$\overline{22}$ & 2 & 3 & 3 & 3 & 1 & 4 & 4 & 4 & 2 & 4 & 4 & 2 & 4 & 2 & 0 \\
$\overline{21}$ & 2 & 3 & 3 & 3 & 1 & 4 & 4 & 4 & 2 & 4 & 4 & 2 & 4 & 2 & 2 \\
$\overline{11}$ & 2 & 3 & 3 & 3 & 3 & 4 & 4 & 4 & 4 & 4 & 4 & 4 & 4 & 4 & 4 \\
\hline
\end{tabular}

Table 16: $B^{1,1} \otimes B^{1,1}$ for $D_{3}^{(2)}$

\begin{tabular}{c|cccccc}
\hline \multirow{2}{*}{$b$} & \multicolumn{6}{|c}{$c$} \\
& 0 & 1 & 2 & $\overline{2}$ & $\overline{1}$ & $\phi$ \\
\hline 0 & 0 & 2 & 2 & 0 & 0 & 1 \\
1 & 0 & 2 & 0 & 0 & 0 & 1 \\
2 & 0 & 2 & 2 & 0 & 0 & 1 \\
$\overline{2}$ & 2 & 2 & 2 & 2 & 0 & 1 \\
$\overline{1}$ & 2 & 2 & 2 & 2 & 2 & 1 \\
$\phi$ & 1 & 1 & 1 & 1 & 1 & 0 \\
\hline
\end{tabular}


Table $17: B^{1,2} \otimes B^{1,1}$ for $D_{3}^{(2)}$

\begin{tabular}{c|cccccc}
\hline \multirow{2}{*}{$b$} & 0 & 1 & 2 & $\overline{2}$ & $\overline{1}$ & $\phi$ \\
\hline 10 & $0 \cdot 10_{0}$ & $0 \cdot 11_{2}$ & $1 \cdot 20_{0}$ & $0 \cdot 1 \overline{2}_{0}$ & $0 \cdot \phi_{0}$ & $0 \cdot 1_{1}$ \\
20 & $0 \cdot 20_{0}$ & $0 \cdot 12_{2}$ & $0 \cdot 22_{2}$ & $0 \cdot 1 \overline{1}_{0}$ & $0 \cdot 2 \overline{1}_{0}$ & $0 \cdot 2_{1}$ \\
$0 \overline{2}$ & $0 \cdot 0 \overline{2}_{0}$ & $\overline{2} \cdot 10_{2}$ & $\overline{2} \cdot 20_{2}$ & $0 \cdot \overline{22}_{0}$ & $\overline{2} \cdot 0 \overline{1}_{0}$ & $\overline{2} \cdot 0_{1}$ \\
$0 \overline{1}$ & $0 \cdot 0 \overline{1}_{0}$ & $\overline{1} \cdot 10_{2}$ & $\overline{1} \cdot 20_{2}$ & $0 \cdot \overline{21}_{0}$ & $0 \cdot \overline{11}_{0}$ & $\overline{1} \cdot 0_{1}$ \\
11 & $1 \cdot 10_{0}$ & $1 \cdot 11_{2}$ & $1 \cdot 12_{0}$ & $1 \cdot 1 \overline{2}_{0}$ & $1 \cdot \phi_{0}$ & $1 \cdot 1_{1}$ \\
12 & $2 \cdot 10_{0}$ & $2 \cdot 11_{2}$ & $1 \cdot 22_{0}$ & $2 \cdot 1 \overline{2}_{0}$ & $2 \cdot \phi_{0}$ & $2 \cdot 1_{1}$ \\
$1 \overline{2}$ & $1 \cdot 0 \overline{2}_{0}$ & $\overline{2} \cdot 11_{2}$ & $1 \cdot 2 \overline{2}_{0}$ & $1 \cdot \overline{22}_{0}$ & $\overline{2} \cdot \phi_{0}$ & $\overline{2} \cdot 1_{1}$ \\
$1 \overline{1}$ & $2 \cdot 0 \overline{2}_{0}$ & $\overline{1} \cdot 11_{2}$ & $2 \cdot 2 \overline{2}_{0}$ & $2 \cdot \overline{22}_{0}$ & $\overline{1} \cdot \phi_{0}$ & $\overline{1} \cdot 1_{1}$ \\
22 & $2 \cdot 20_{0}$ & $2 \cdot 12_{2}$ & $2 \cdot 22_{2}$ & $2 \cdot 1 \overline{1}_{0}$ & $2 \cdot 2 \overline{1}_{0}$ & $2 \cdot 2_{1}$ \\
$2 \overline{2}$ & $0 \cdot 2 \overline{2}_{0}$ & $\overline{2} \cdot 12_{2}$ & $\overline{2} \cdot 22_{2}$ & $\overline{2} \cdot 1 \overline{1}_{0}$ & $\overline{2} \cdot 2 \overline{1}_{0}$ & $\overline{2} \cdot 2_{1}$ \\
$2 \overline{1}$ & $2 \cdot 0 \overline{1}_{0}$ & $\overline{1} \cdot 12_{2}$ & $\overline{1} \cdot 22_{2}$ & $2 \cdot \overline{21}_{0}$ & $2 \cdot \overline{11}_{0}$ & $\overline{1} \cdot 2_{1}$ \\
$\overline{22}$ & $\overline{2} \cdot 0 \overline{2}_{2}$ & $\overline{2} \cdot 1 \overline{2}_{2}$ & $\overline{2} \cdot 2 \overline{2}_{2}$ & $\overline{2} \cdot \overline{22}_{2}$ & $\overline{2} \cdot \overline{21}_{0}$ & $\overline{2} \cdot \overline{2}_{1}$ \\
$\overline{21}$ & $\overline{1} \cdot 0 \overline{2}_{2}$ & $\overline{1} \cdot 1 \overline{2}_{2}$ & $\overline{1} \cdot 2 \overline{2}_{2}$ & $\overline{1} \cdot \overline{22}_{2}$ & $\overline{2} \cdot \overline{11}_{0}$ & $\overline{1} \cdot \overline{2}_{1}$ \\
$\overline{11}$ & $\overline{1} \cdot 0 \overline{1}_{2}$ & $\overline{1} \cdot 1 \overline{1}_{2}$ & $\overline{1} \cdot 2 \overline{1}_{2}$ & $\overline{1} \cdot \overline{21}_{2}$ & $\overline{1} \cdot \overline{11}_{2}$ & $\overline{1} \cdot \overline{1}_{1}$ \\
0 & $0 \cdot 0_{0}$ & $\phi \cdot 10_{1}$ & $\phi \cdot 20_{1}$ & $0 \cdot \overline{2}_{0}$ & $0 \cdot \overline{1}_{0}$ & $\phi \cdot 0_{0}$ \\
1 & $1 \cdot 0_{0}$ & $\phi \cdot 11_{1}$ & $1 \cdot 2_{0}$ & $1 \cdot \overline{2}_{0}$ & $1 \cdot \overline{1}_{0}$ & $\phi \cdot 1_{0}$ \\
2 & $2 \cdot 0_{0}$ & $\phi \cdot 12_{1}$ & $\phi \cdot 22_{1}$ & $2 \cdot \overline{2}_{0}$ & $2 \cdot \overline{1}_{0}$ & $\phi \cdot 2_{0}$ \\
$\overline{2}$ & $\phi \cdot 0 \overline{2}_{1}$ & $\phi \cdot 1 \overline{2}_{1}$ & $\phi \cdot 2 \overline{2}_{1}$ & $\phi \cdot \overline{22}_{1}$ & $\overline{2} \cdot \overline{1}_{0}$ & $\phi \cdot \overline{2}_{0}$ \\
$\overline{1}$ & $\phi \cdot 0 \overline{1}_{1}$ & $\phi \cdot 1 \overline{1}_{1}$ & $\phi \cdot 2 \overline{1}_{1}$ & $\phi \cdot \overline{21}_{1}$ & $\phi \cdot \overline{11}_{1}$ & $\phi \cdot \overline{1}_{0}$ \\
$\phi$ & $1 \cdot 0 \overline{1}_{0}$ & $1 \cdot 1 \overline{1}_{0}$ & $1 \cdot 2 \overline{1}_{0}$ & $1 \cdot \overline{21}_{0}$ & $1 \cdot \overline{11}_{0}$ & $\phi \cdot \phi_{0}$ \\
\hline
\end{tabular}


Table 18: $B^{1,2} \otimes B^{1,2}$ for $D_{3}^{(2)}$

\begin{tabular}{c|ccccccccccccccccccccc}
\hline \multirow{2}{*}{$b$} & 10 & 20 & $0 \overline{2}$ & $0 \overline{1}$ & 11 & 12 & $1 \overline{2}$ & $1 \overline{1}$ & 22 & $2 \overline{2}$ & $2 \overline{1}$ & $\overline{22}$ & $\overline{21}$ & $\overline{11}$ & 0 & 1 & 2 & $\overline{2}$ & $\overline{1}$ & $\phi$ \\
\hline 10 & 2 & 0 & 0 & 0 & 4 & 2 & 2 & 0 & 2 & 0 & 0 & 0 & 0 & 0 & 1 & 3 & 1 & 1 & 1 & 2 \\
20 & 2 & 2 & 0 & 0 & 4 & 4 & 2 & 2 & 4 & 0 & 2 & 0 & 0 & 0 & 1 & 3 & 3 & 1 & 1 & 2 \\
$0 \overline{2}$ & 2 & 2 & 2 & 0 & 4 & 4 & 2 & 2 & 4 & 2 & 2 & 2 & 0 & 0 & 1 & 3 & 3 & 1 & 1 & 2 \\
$0 \overline{1}$ & 2 & 2 & 2 & 2 & 4 & 4 & 2 & 2 & 4 & 2 & 2 & 2 & 2 & 2 & 1 & 3 & 3 & 1 & 1 & 2 \\
11 & 2 & 0 & 0 & 0 & 4 & 2 & 2 & 0 & 0 & 0 & 0 & 0 & 0 & 0 & 1 & 3 & 1 & 1 & 1 & 2 \\
12 & 2 & 0 & 0 & 0 & 4 & 2 & 2 & 0 & 2 & 0 & 0 & 0 & 0 & 0 & 1 & 3 & 1 & 1 & 1 & 2 \\
$1 \overline{2}$ & 2 & 2 & 2 & 0 & 4 & 2 & 2 & 0 & 2 & 2 & 0 & 2 & 0 & 0 & 1 & 3 & 1 & 1 & 1 & 2 \\
$1 \overline{1}$ & 2 & 2 & 2 & 0 & 4 & 2 & 2 & 0 & 2 & 2 & 0 & 2 & 0 & 0 & 1 & 3 & 1 & 1 & 1 & 2 \\
22 & 2 & 2 & 0 & 0 & 4 & 4 & 2 & 2 & 4 & 0 & 2 & 0 & 0 & 0 & 1 & 3 & 3 & 1 & 1 & 2 \\
$2 \overline{2}$ & 2 & 2 & 0 & 0 & 4 & 4 & 2 & 2 & 4 & 0 & 2 & 0 & 0 & 0 & 1 & 3 & 3 & 1 & 1 & 2 \\
$2 \overline{1}$ & 2 & 2 & 2 & 2 & 4 & 4 & 2 & 2 & 4 & 2 & 2 & 2 & 2 & 2 & 1 & 3 & 3 & 1 & 1 & 2 \\
$\overline{22}$ & 4 & 4 & 4 & 2 & 4 & 4 & 4 & 2 & 4 & 4 & 2 & 4 & 2 & 0 & 3 & 3 & 3 & 3 & 1 & 2 \\
$\overline{21}$ & 4 & 4 & 4 & 2 & 4 & 4 & 4 & 2 & 4 & 4 & 2 & 4 & 2 & 2 & 3 & 3 & 3 & 3 & 1 & 2 \\
$\overline{11}$ & 4 & 4 & 4 & 4 & 4 & 4 & 4 & 4 & 4 & 4 & 4 & 4 & 4 & 4 & 3 & 3 & 3 & 3 & 3 & 2 \\
0 & 1 & 1 & 1 & 1 & 3 & 3 & 1 & 1 & 3 & 1 & 1 & 1 & 1 & 1 & 0 & 2 & 2 & 0 & 0 & 1 \\
1 & 1 & 1 & 1 & 1 & 3 & 1 & 1 & 1 & 1 & 1 & 1 & 1 & 1 & 1 & 0 & 2 & 0 & 0 & 0 & 1 \\
2 & 1 & 1 & 1 & 1 & 3 & 3 & 1 & 1 & 3 & 1 & 1 & 1 & 1 & 1 & 0 & 2 & 2 & 0 & 0 & 1 \\
$\overline{2}$ & 3 & 3 & 3 & 1 & 3 & 3 & 3 & 1 & 3 & 3 & 1 & 3 & 1 & 1 & 2 & 2 & 2 & 2 & 0 & 1 \\
$\overline{1}$ & 3 & 3 & 3 & 3 & 3 & 3 & 3 & 3 & 3 & 3 & 3 & 3 & 3 & 3 & 2 & 2 & 2 & 2 & 2 & 1 \\
$\phi$ & 2 & 2 & 2 & 2 & 2 & 2 & 2 & 2 & 2 & 2 & 2 & 2 & 2 & 2 & 1 & 1 & 1 & 1 & 1 & 0 \\
\hline
\end{tabular}

Table 19: $B^{1,1} \otimes B^{1,1}$ for $D_{4}^{(3)}$

\begin{tabular}{|c|c|c|c|c|c|c|c|c|}
\hline \multirow[b]{2}{*}{$b$} & \multicolumn{8}{|c|}{$c$} \\
\hline & $\phi$ & $\overline{1}$ & $\overline{2}$ & $\overline{3}$ & 0 & 3 & 2 & 1 \\
\hline$\phi$ & 0 & 1 & 1 & 1 & 1 & 1 & 1 & 1 \\
\hline$\overline{1}$ & 1 & 2 & 2 & 2 & 2 & 2 & 2 & 2 \\
\hline$\overline{2}$ & 1 & 1 & 2 & 2 & 2 & 2 & 2 & 2 \\
\hline$\overline{3}$ & 1 & 1 & 1 & 2 & 2 & 2 & 2 & 2 \\
\hline 0 & 1 & 0 & 1 & 1 & 1 & 2 & 2 & 2 \\
\hline 3 & 1 & 0 & 1 & 1 & 1 & 2 & 2 & 2 \\
\hline 2 & 1 & 0 & 0 & 1 & 1 & 1 & 2 & 2 \\
\hline 1 & 1 & 0 & 0 & 0 & 0 & 1 & 1 & 2 \\
\hline
\end{tabular}


Table 20: $B^{1,2} \otimes B^{1,1}$ for $D_{4}^{(3)}$

\begin{tabular}{|c|c|c|c|c|c|c|c|c|}
\hline \multirow[b]{2}{*}{$b$} & \multicolumn{8}{|c|}{$c$} \\
\hline & $\phi$ & $\overline{1}$ & $\overline{2}$ & $\overline{3}$ & 0 & 3 & 2 & 1 \\
\hline$\overline{11}$ & $\overline{1} \cdot \overline{1}_{1}$ & $\overline{1} \cdot \overline{11}_{2}$ & $\overline{1} \cdot \overline{21}_{2}$ & $\overline{1} \cdot \overline{31}_{2}$ & $\overline{1} \cdot 0 \overline{1}_{2}$ & $\overline{1} \cdot 3 \overline{1}_{2}$ & $\overline{1} \cdot 2 \overline{1}_{2}$ & $\overline{1} \cdot 1 \overline{1}_{2}$ \\
\hline$\overline{33}$ & $\overline{3} \cdot \overline{3}_{1}$ & $\overline{3} \cdot \overline{31}_{1}$ & $\overline{3} \cdot 0 \overline{1}_{1}$ & $\overline{3} \cdot \overline{33}_{2}$ & $\overline{3} \cdot 0 \overline{3}_{2}$ & $\overline{3} \cdot 3 \overline{3}_{2}$ & $\overline{3} \cdot 2 \overline{3}_{2}$ & $\overline{3} \cdot 1 \overline{3}_{2}$ \\
\hline 20 & $0 \cdot 2_{1}$ & $0 \cdot \overline{3}_{0}$ & $0 \cdot 0_{0}$ & $0 \cdot 2 \overline{3}_{1}$ & $0 \cdot 1 \overline{3}_{1}$ & $0 \cdot 10_{1}$ & $0 \cdot 22_{2}$ & $0 \cdot 12_{2}$ \\
\hline$\overline{22}$ & $\overline{2} \cdot \overline{2}_{1}$ & $\overline{2} \cdot \overline{21}_{1}$ & $\overline{2} \cdot \overline{22}_{2}$ & $\overline{2} \cdot \overline{32}_{2}$ & $\overline{2} \cdot 0 \overline{2}_{2}$ & $\overline{2} \cdot 3 \overline{2}_{2}$ & $\overline{2} \cdot 2 \overline{2}_{2}$ & $\overline{2} \cdot 1 \overline{2}_{2}$ \\
\hline$\overline{32}$ & $\overline{2} \cdot \overline{3}_{1}$ & $\overline{2} \cdot \overline{31}_{1}$ & $\overline{2} \cdot 0 \overline{1}_{1}$ & $\overline{2} \cdot \overline{33}_{2}$ & $\overline{2} \cdot 0 \overline{3}_{2}$ & $\overline{2} \cdot 3 \overline{3}_{2}$ & $\overline{2} \cdot 2 \overline{3}_{2}$ & $\overline{2} \cdot 1 \overline{3}_{2}$ \\
\hline $0 \overline{2}$ & $\overline{2} \cdot 0_{1}$ & $\overline{2} \cdot \overline{1}_{0}$ & $\overline{2} \cdot 3 \overline{1}_{1}$ & $\overline{2} \cdot 2 \overline{1}_{1}$ & $\overline{2} \cdot 1 \overline{1}_{1}$ & $\overline{2} \cdot 30_{2}$ & $\overline{2} \cdot 20_{2}$ & $\overline{2} \cdot 10_{2}$ \\
\hline $0 \overline{3}$ & $\overline{3} \cdot 0_{1}$ & $\overline{3} \cdot \overline{1}_{0}$ & $\overline{3} \cdot 3 \overline{1}_{1}$ & $\overline{3} \cdot 2 \overline{1}_{1}$ & $\overline{3} \cdot 1 \overline{1}_{1}$ & $\overline{3} \cdot 30_{2}$ & $\overline{3} \cdot 20_{2}$ & $\overline{3} \cdot 10_{2}$ \\
\hline $3 \overline{3}$ & $\overline{3} \cdot 3_{1}$ & $\overline{3} \cdot \overline{2}_{0}$ & $\overline{3} \cdot 3 \overline{2}_{1}$ & $\overline{3} \cdot 2 \overline{2}_{1}$ & $\overline{3} \cdot 1 \overline{2}_{1}$ & $\overline{3} \cdot 33_{2}$ & $\overline{3} \cdot 23_{2}$ & $\overline{3} \cdot 13_{2}$ \\
\hline 30 & $0 \cdot 3_{1}$ & $0 \cdot \overline{2}_{0}$ & $0 \cdot 3 \overline{2}_{1}$ & $0 \cdot 2 \overline{2}_{1}$ & $0 \cdot 1 \overline{2}_{1}$ & $0 \cdot 33_{2}$ & $0 \cdot 23_{2}$ & $0 \cdot 13_{2}$ \\
\hline 33 & $3 \cdot 3_{1}$ & $3 \cdot \overline{2}_{0}$ & $3 \cdot 3 \overline{2}_{1}$ & $3 \cdot 2 \overline{2}_{1}$ & $3 \cdot 1 \overline{2}_{1}$ & $3 \cdot 33_{2}$ & $3 \cdot 23_{2}$ & $3 \cdot 13_{2}$ \\
\hline 23 & $3 \cdot 2_{1}$ & $3 \cdot \overline{3}_{0}$ & $3 \cdot 0_{0}$ & $3 \cdot 2 \overline{3}_{1}$ & $3 \cdot 1 \overline{3}_{1}$ & $3 \cdot 10_{1}$ & $3 \cdot 22_{2}$ & $3 \cdot 12_{2}$ \\
\hline 22 & $2 \cdot 2_{1}$ & $2 \cdot \overline{3}_{0}$ & $2 \cdot 0_{0}$ & $2 \cdot 2 \overline{3}_{1}$ & $2 \cdot 1 \overline{3}_{1}$ & $2 \cdot 10_{1}$ & $2 \cdot 22_{2}$ & $2 \cdot 12_{2}$ \\
\hline $3 \overline{2}$ & $\overline{2} \cdot 3_{1}$ & $3 \cdot \overline{21}_{0}$ & $3 \cdot \overline{22}_{1}$ & $3 \cdot \overline{32}_{1}$ & $3 \cdot 0 \overline{2}_{1}$ & $\overline{2} \cdot 33_{2}$ & $\overline{2} \cdot 23_{2}$ & $\overline{2} \cdot 13_{2}$ \\
\hline $2 \overline{2}$ & $\overline{2} \cdot 2_{1}$ & $3 \cdot \overline{31}_{0}$ & $3 \cdot 0 \overline{1}_{0}$ & $3 \cdot \overline{33}_{1}$ & $3 \cdot 0 \overline{3}_{1}$ & $3 \cdot 3 \overline{3}_{1}$ & $\overline{2} \cdot 22_{2}$ & $\overline{2} \cdot 12_{2}$ \\
\hline $2 \overline{3}$ & $\overline{3} \cdot 2_{1}$ & $2 \cdot \overline{31}_{0}$ & $2 \cdot 0 \overline{1}_{0}$ & $2 \cdot \overline{33}_{1}$ & $2 \cdot 0 \overline{3}_{1}$ & $2 \cdot 3 \overline{3}_{1}$ & $\overline{3} \cdot 22_{2}$ & $\overline{3} \cdot 12_{2}$ \\
\hline$\overline{21}$ & $\overline{1} \cdot \overline{2}_{1}$ & $\overline{2} \cdot \overline{11}_{1}$ & $\overline{1} \cdot \overline{22}_{2}$ & $\overline{1} \cdot \overline{32}_{2}$ & $\overline{1} \cdot 0 \overline{2}_{2}$ & $\overline{1} \cdot 3 \overline{2}_{2}$ & $\overline{1} \cdot 2 \overline{2}_{2}$ & $\overline{1} \cdot 1 \overline{2}_{2}$ \\
\hline$\overline{31}$ & $\overline{1} \cdot \overline{3}_{1}$ & $\overline{3} \cdot \overline{11}_{1}$ & $\overline{3} \cdot \overline{21}_{1}$ & $\overline{1} \cdot \overline{33}_{2}$ & $\overline{1} \cdot 0 \overline{3}_{2}$ & $\overline{1} \cdot 3 \overline{3}_{2}$ & $\overline{1} \cdot 2 \overline{3}_{2}$ & $\overline{1} \cdot 1 \overline{3}_{2}$ \\
\hline $0 \overline{1}$ & $\overline{1} \cdot 0_{1}$ & $0 \cdot \overline{11}_{0}$ & $\overline{3} \cdot \overline{22}_{1}$ & $\overline{3} \cdot \overline{32}_{1}$ & $\overline{3} \cdot 0 \overline{2}_{1}$ & $\overline{1} \cdot 30_{2}$ & $\overline{1} \cdot 20_{2}$ & $\overline{1} \cdot 10_{2}$ \\
\hline $3 \overline{1}$ & $\overline{1} \cdot 3_{1}$ & $0 \cdot \overline{21}_{0}$ & $0 \cdot \overline{22}_{1}$ & $0 \cdot \overline{32}_{1}$ & $0 \cdot 0 \overline{2}_{1}$ & $\overline{1} \cdot 33_{2}$ & $\overline{1} \cdot 23_{2}$ & $\overline{1} \cdot 13_{2}$ \\
\hline $2 \overline{1}$ & $\overline{1} \cdot 2_{1}$ & $0 \cdot \overline{31}_{0}$ & $0 \cdot 0 \overline{1}_{0}$ & $0 \cdot \overline{33}_{1}$ & $0 \cdot 0 \overline{3}_{1}$ & $0 \cdot 3 \overline{3}_{1}$ & $\overline{1} \cdot 22_{2}$ & $\overline{1} \cdot 12_{2}$ \\
\hline $1 \overline{1}$ & $\overline{1} \cdot 1_{1}$ & $\overline{1} \cdot \phi_{0}$ & $0 \cdot 3 \overline{1}_{0}$ & $0 \cdot 2 \overline{1}_{0}$ & $0 \cdot 1 \overline{1}_{0}$ & $0 \cdot 30_{1}$ & $0 \cdot 20_{1}$ & $\overline{1} \cdot 11_{2}$ \\
\hline $1 \overline{2}$ & $\overline{2} \cdot 1_{1}$ & $\overline{2} \cdot \phi_{0}$ & $3 \cdot 3 \overline{1}_{0}$ & $3 \cdot 2 \overline{1}_{0}$ & $3 \cdot 1 \overline{1}_{0}$ & $3 \cdot 30_{1}$ & $3 \cdot 20_{1}$ & $\overline{2} \cdot 11_{2}$ \\
\hline $1 \overline{3}$ & $\overline{3} \cdot 1_{1}$ & $\overline{3} \cdot \phi_{0}$ & $2 \cdot 3 \overline{1}_{0}$ & $2 \cdot 2 \overline{1}_{0}$ & $2 \cdot 1 \overline{1}_{0}$ & $2 \cdot 30_{1}$ & $2 \cdot 20_{1}$ & $\overline{3} \cdot 11_{2}$ \\
\hline 10 & $0 \cdot 1_{1}$ & $0 \cdot \phi_{0}$ & $2 \cdot 3 \overline{2}_{0}$ & $2 \cdot 2 \overline{2}_{0}$ & $2 \cdot 1 \overline{2}_{0}$ & $2 \cdot 33_{1}$ & $2 \cdot 23_{1}$ & $0 \cdot 11_{2}$ \\
\hline 13 & $3 \cdot 1_{1}$ & $3 \cdot \phi_{0}$ & $1 \cdot 3 \overline{2}_{0}$ & $1 \cdot 2 \overline{2}_{0}$ & $1 \cdot 1 \overline{2}_{0}$ & $1 \cdot 33_{1}$ & $1 \cdot 23_{1}$ & $3 \cdot 11_{2}$ \\
\hline 12 & $2 \cdot 1_{1}$ & $2 \cdot \phi_{0}$ & $2 \cdot 3_{0}$ & $1 \cdot 2 \overline{3}_{0}$ & $1 \cdot 1 \overline{3}_{0}$ & $2 \cdot 13_{1}$ & $1 \cdot 22_{1}$ & $2 \cdot 11_{2}$ \\
\hline 11 & $1 \cdot 1_{1}$ & $1 \cdot \phi_{0}$ & $1 \cdot 3_{0}$ & $1 \cdot 2_{0}$ & $1 \cdot 10_{0}$ & $1 \cdot 13_{1}$ & $1 \cdot 12_{1}$ & $1 \cdot 11_{2}$ \\
\hline$\phi$ & $\phi \cdot \phi_{0}$ & $1 \cdot \overline{11}_{0}$ & $1 \cdot \overline{21}_{0}$ & $1 \cdot \overline{31}_{0}$ & $1 \cdot 0 \overline{1}_{0}$ & $1 \cdot 3 \overline{1}_{0}$ & $1 \cdot 2 \overline{1}_{0}$ & $1 \cdot 1 \overline{1}_{0}$ \\
\hline$\overline{1}$ & $\phi \cdot \overline{1}_{0}$ & $\phi \cdot \overline{11}_{1}$ & $\phi \cdot \overline{21}_{1}$ & $\phi \cdot \overline{31}_{1}$ & $\phi \cdot 0 \overline{1}_{1}$ & $\phi \cdot 3 \overline{1}_{1}$ & $\phi \cdot 2 \overline{1}_{1}$ & $\phi \cdot 1 \overline{1}_{1}$ \\
\hline$\overline{2}$ & $\phi \cdot \overline{2}_{0}$ & $3 \cdot \overline{11}_{0}$ & $\phi \cdot \overline{22}_{1}$ & $\phi \cdot \overline{32}_{1}$ & $\phi \cdot 0 \overline{2}_{1}$ & $\phi \cdot 3 \overline{2}_{1}$ & $\phi \cdot 2 \overline{2}_{1}$ & $\phi \cdot 1 \overline{2}_{1}$ \\
\hline$\overline{3}$ & $\phi \cdot \overline{3}_{0}$ & $2 \cdot \overline{11}_{0}$ & $2 \cdot \overline{21}_{0}$ & $\phi \cdot \overline{33}_{1}$ & $\phi \cdot 0 \overline{3}_{1}$ & $\phi \cdot 3 \overline{3}_{1}$ & $\phi \cdot 2 \overline{3}_{1}$ & $\phi \cdot 1 \overline{3}_{1}$ \\
\hline 0 & $\phi \cdot 0_{0}$ & $0 \cdot \overline{1}_{0}$ & $2 \cdot \overline{22}_{0}$ & $2 \cdot \overline{32}_{0}$ & $2 \cdot 0 \overline{2}_{0}$ & $\phi \cdot 30_{1}$ & $\phi \cdot 20_{1}$ & $\phi \cdot 10_{1}$ \\
\hline 3 & $\phi \cdot 3_{0}$ & $3 \cdot \overline{1}_{0}$ & $1 \cdot \overline{22}_{0}$ & $1 \cdot \overline{32}_{0}$ & $1 \cdot 0 \overline{2}_{0}$ & $\phi \cdot 33_{1}$ & $\phi \cdot 23_{1}$ & $\phi \cdot 13_{1}$ \\
\hline 2 & $\phi \cdot 2_{0}$ & $2 \cdot \overline{1}_{0}$ & $2 \cdot \overline{2}_{0}$ & $1 \cdot \overline{33}_{0}$ & $1 \cdot 0 \overline{3}_{0}$ & $1 \cdot 3 \overline{3}_{0}$ & $\phi \cdot 22_{1}$ & $\phi \cdot 12_{1}$ \\
\hline 1 & $\phi \cdot 1_{0}$ & $1 \cdot \overline{1}_{0}$ & $1 \cdot \overline{2}_{0}$ & $1 \cdot \overline{3}_{0}$ & $1 \cdot 0_{0}$ & $1 \cdot 30_{0}$ & $1 \cdot 20_{0}$ & $\phi \cdot 11_{1}$ \\
\hline
\end{tabular}




\section{References}

[AM] S. R. Aladim and M. J. Martins, Critical behaviour of integrable mixed spin chains, J. Phys. A26 (1993) L529-L534.

$[\mathrm{ABF}]$ G. E. Andrews, R. J. Baxter and P. J. Forrester, Eight vertex SOS model and generalized Rogers-Ramanujan-type identities, J. Stat. Phys. 35, (1984) $193-266$.

[AI] K. Aomoto and K. Iguchi, Wu's equations and quasi-hypergeometric functions, preprint.

[ANOT] T. Arakawa, T. Nakanishi, K. Oshima and A. Tsuchiya, Spectral decomposition of path space in solvable lattice model, Commun. Math. Phys. 181 (1996) 159-182.

[B] R.J. Baxter, Exactly solved models in statistical mechanics, Academic Press, London (1982).

[Ber] A. Berkovich, Fermionic counting of RSOS-states and Virasoro character formulas for the unitary minimal series $M(\nu, \nu+1)$. Exact results, Nucl. Phys. B431 (1994) 315-348.

[BM] A. Berkovich, B. M. McCoy, The universal chiral partition function for exclusion statistics, in Statistical physics on the eve of the 21st century, Ed. by M. T. Batchelor and L.T. Wille, Ser. Adv. Stat. Mech. 14 World Sci. Publ. River Edge, NJ (1999) 240-256.

[BMS] A. Berkovich, B. M. McCoy and A. Schilling, Rogers-Schur-Ramanujan type identities for the $M\left(p, p^{\prime}\right)$ minimal models of conformal field theory, Commun.Math.Phys. 191 (1998) 325-395.

[BMSW] A. Berkovich, B.M. McCoy, A. Schilling and S.O. Warnaar, Bailey flows and Bose-Fermi identities for the conformal coset models $\left(A_{1}^{(1)}\right)_{N} \times$ $\left(A_{1}^{(1)}\right)_{N^{\prime}} /\left(A_{1}^{(1)}\right)_{N+N^{\prime}}$, Nucl.Phys. B499 (1997) 621-649.

[BPS] D. Bernard, V. Pasquier and D. Serban, Spinons in Conformal Field Theory, Nucl. Phys. B428 (1994) 612-628.

[Be] H. A. Bethe, Zur Theorie der Metalle, I. Eigenwerte und Eigenfunktionen der linearen Atomkette, Z. Physik 71 (1931) 205-231.

[BLS1] P. Bouwknegt, A.W.W. Ludwig and K. Schoutens, Spinon Bases, Yangian Symmetry and Fermionic Representations of Virasoro Characters in Conformal Field Theory, Phys. Lett. B338 (1994) 448-456.

[BLS2] P. Bouwknegt, A.W.W. Ludwig and K. Schoutens, Spinon basis for higher level SU(2) WZW models, Phys.Lett. B359 (1995) 304-312. 
[BS1] P. Bouwknegt and K. Schoutens, Spinon decomposition and Yangian structure of $\widehat{s l}_{n}$ modules, in "Geometric Analysis and Lie Theory in Mathematics and Physics", Austral. Math. Soc. Lect. Ser. 11, Cambridge Univ. Press, Cambridge (1998) 105-131.

[BS2] P. Bouwknegt and K. Schoutens, Exclusion statistics in conformal field theory - generalized fermions and spinons for level-1 WZW theories, Nucl. Phys. B547 (1999) 501-537.

[C1] V. Chari, On the fermionic formula and the Kirillov-Reshetikhin conjecture, math.QA/0006090.

[C2] V. Chari, Tensor products of level zero representations, math.QA/0012116.

[CP1] V. Chari and A. Pressley, Quantum affine algebras and their representations, in Representations of groups, CMS Conf. Proc. 16, Amer. Math. Soc., Providence, RI (1995) 59-78.

[CP2] V. Chari and A. Pressley, Twisted quantum affine algebras, Commun. Math. Phys. 196 (1998) 461-476.

[DKKMM] S. Dasmahapatra, R. Kedem, T.R. Klassen, B.M. McCoy and E. Melzer, Quasi-Particles, Conformal Field Theory, and q-Series, Int. J. Mod. Phys. B7 (1993) 3617-3648.

[DJKMO1] E. Date, M. Jimbo, A. Kuniba, T. Miwa and M. Okado, Exactly solvable SOS models II: Proof of the star-triangle relation and combinatorial identities, Adv. Stud. Pure Math. 16 (1988) 17-122.

[DJKMO2] E. Date, M. Jimbo, A. Kuniba, T. Miwa and M. Okado, One dimensional configuration sums in vertex models and affine Lie algebra characters, Lett. Math. Phys. 17 (1989) 69-77.

[DJO] E. Date, M. Jimbo and M. Okado, Crystal base and q-vertex operators, Commun. Math. Phys. 155 (1993) 47-69.

[DMN] H. J. de Vega, L. Mezincescu, and R. I. Nepomechie, Thermodynamics of integrable chains with alternating spins, Phys. Rev. B49 (1994) 1322313226 .

[FKLMM] B. Feigin R. Kedem, S. Loktev, T. Miwa and E. Mukhin, Combinatorics of the $\widehat{s l}_{2}$ spaces of coinvariants III, math.QA/0012190.

[FS] B. L. Feigin and A. V. Stoyanovsky, Quasi-particle models for the representations of Lie algebras and geometry of flag manifold, hep-th/9308079.

[FLOTW] O. Foda, B. Leclerc, M. Okado, J.-Y. Thibon and T. Welsh, Combinatorics of solvable lattice models, and modular representations of Hecke algebras, in "Geometric Analysis and Lie Theory in Mathematics and Physics", Austral. Math. Soc. Lect. Ser. 11, Cambridge Univ. Press, Cambridge (1998) 243-290. 
[FOW] O. Foda, M. Okado and O. Warnaar, A proof of polynomial identities of type $\widehat{s l(n)_{1}} \otimes \widehat{s l(n)_{1}} / \widehat{s l(n)_{2}}$, J. Math. Phys. 37 (1996) 965-986.

[FW1] O. Foda and T. A. Welsh, Melzer's identities revisited, Contemporary Math. 248 (1999) 207-234.

[FW2] O. Foda and T. A. Welsh, On the combinatorics of Forrester-Baxter models, in "Physical Combinatorics" Prog. in Math. (M. Kashiwara and T. Miwa ed. Birkhäuser, 2000) 49-103.

[FB] P. J. Forrester and R. J. Baxter, Further exact solutions of the eight-vertex SOS model and generalizations of the Rogers-Ramanujan identities, J. Stat. Phys. 38 (1985) 435-472.

[G] G. Georgiev, Combinatorial constructions of modules for infinitedimensional Lie algebras, II. Parafermionic space, q-alg/9504024.

[HKKOT] G. Hatayama, Y. Koga, A. Kuniba, M. Okado and T. Takagi, Finite crystals and paths, Adv. Stud. in Pure Math. 28 (2000) 113-132.

[HKKOTY] G. Hatayama, A. N. Kirillov, A. Kuniba, M. Okado, T. Takagi, Y. Yamada, Character formulae of $\widehat{s l}_{n}$-modules and inhomogeneous paths, Nucl. Phys. B536 [PM] (1999) 575-616.

[HKOT1] G. Hatayama, A. Kuniba, M. Okado and T. Takagi, Combinatorial $R$ matrices for a family of crystals: $C_{n}^{(1)}$ and $A_{2 n-1}^{(2)}$ cases, in "Physical Combinatorics" Prog. in Math. (M. Kashiwara and T. Miwa ed. Birkhäuser, 2000) 105-139.

[HKOT2] G. Hatayama, A. Kuniba, M. Okado and T. Takagi, Combinatorial $R$ matrices for a family of crystals : $B_{n}^{(1)}, D_{n}^{(1)}, A_{2 n}^{(2)}$ and $D_{n+1}^{(2)}$ cases, math.QA/0012247.

[HKOTY1] G. Hatayama, A. Kuniba, M. Okado, T. Takagi, Y. Yamada, Remarks on fermionic formula, Contemporary Math. 248 (1999) 243-291.

[HKOTY2] G. Hatayama, A. Kuniba, M. Okado, T. Takagi, Y. Yamada, Scattering rules in soliton cellular automata associated with crystal bases, math.QA/0007175, to appear in Contemporary Math.

[HKMW] J. Hong, S-J. Kang, T. Miwa, R. Weston, Mixing of ground states in vertex models, J. Phys. A31 (1998) L515-L525.

[JMMO] M. Jimbo, K. C. Misra, T. Miwa and M. Okado, Combinatorics of representations of $U_{q}(\widehat{s l}(n))$ at $q=0$, Commun. Math. Phys. 136 (1991) $543-566$.

[JMO] M. Jimbo, T. Miwa and M. Okado, Local state probabilities of solvable lattice models: an $A_{n-1}^{(1)}$ family, Nucl. Phys. B300[FS22] (1988) 74-108. 
[JnMO] N. Jing, K. C. Misra and M. Okado, q-wedge modules for quantized enveloping algebras of classical type, J. of Alg. 230 (2000) 518-539.

[Kac] V. G. Kac, Infinite dimensional Lie algebras, 3rd edition, Cambridge Univ. Press, Cambridge (1990).

[KK] S-J. Kang and M. Kashiwara, Quantized affine algebras and crystals with core, Commun. Math. Phys. 195 (1998) 725-740.

[KKM] S-J. Kang, M. Kashiwara and K. C. Misra, Crystal bases of Verma modules for quantum affine Lie algebras, Compositio Math. 92 (1994) 299325 .

[KMN1] S-J. Kang, M. Kashiwara, K. C. Misra, T. Miwa, T. Nakashima and A. Nakayashiki, Affine crystals and vertex models, Int. J. Mod. Phys. A7 (suppl. 1A), (1992) 449-484.

[KMN2] S-J. Kang, M. Kashiwara, K. C. Misra, T. Miwa, T. Nakashima and A. Nakayashiki, Perfect crystals of quantum affine Lie algebras, Duke Math. J. 68 (1992) 499-607.

[Ka1] M. Kashiwara, On crystal bases of the q-analogue of universal enveloping algebras, Duke Math. J. 63 (1991) 465-516.

[Ka2] M. Kashiwara, The crystal base and Littelmann's refined Demazure character formula, Duke Math. 71 (1993) 839-858.

[Ka3] M. Kashiwara, On level zero representations of quantized affine algebras, math.QA/0010293.

[KMM] R. Kedem, B.M. McCoy and E. Melzer, The sums of Rogers, Schur and Ramanujan and the Bose-Fermi correspondence in $(1+1)$-dimensional quantum field theory, in Recent progress in statistical mechanics and quantum field theory, World Sci. Publ. River Edge, NJ (1995) 195-219.

[KKMM1] R. Kedem, T.R. Klassen, B.M. McCoy, E. Melzer, Fermionic quasiparticle representations for characters of $\left(G^{(1)}\right)_{1} \times\left(G^{(1)}\right)_{1} /\left(G^{(1)}\right)_{2}$, Phys. Lett. B304 (1993) 263-270.

[KKMM2] R. Kedem, T.R. Klassen, B.M. McCoy, E. Melzer, Fermionic sum representations for conformal field theory characters, Phys. Lett. B307 (1993) 68-76.

[KM] R. Kedem and B. M. McCoy, Construction of Modular Branching Functions from Bethe's Equations in the 3-State Potts Chain, J. Stat. Phys. 71 (1993) 865-901.

[KKR] S. V. Kerov, A. N. Kirillov and N. Yu. Reshetikhin, Combinatorics, the Bethe ansatz and representations of the symmetric group, Zap.Nauchn. Sem. (LOMI) 155 (1986) 50-64. (English translation: J. Sov. Math. 41 (1988) 916-924.) 
[Ki1] A. N. Kirillov, Combinatorial identities and completeness of states for the Heisenberg magnet, J. Sov. Math. 30 (1985) 2298-3310.

[Ki2] A. N. Kirillov, Completeness of states of the generalized Heisenberg magnet, J. Sov. Math. 36 (1987) 115-128.

[Ki3] A. N. Kirillov, Identities for the Rogers dilogarithm function connected with simple Lie algebras, J. Sov. Math. 47 (1989) 2450-2459.

[Ki4] A. N. Kirillov, Dilogarithm identities, Lectures in Mathematical Sciences 7, The University of Tokyo (1995).

[KKN] A. N. Kirillov, A. Kuniba and T. Nakanishi, Skew Young diagram method in spectral decomposition of integrable lattice models, Commun. Math. Phys. 185 (1997) 441-465.

[KR1] A. N. Kirillov and N. Yu. Reshetikhin, The Bethe ansatz and the combinatorics of Young tableaux, J. Sov. Math. 41 (1988) 925-955.

[KR2] A. N. Kirillov and N. Yu. Reshetikhin, Representations of Yangians and multiplicity of occurrence of the irreducible components of the tensor product of representations of simple Lie algebras, J. Sov. Math. 52 (1990) 3156-3164.

[KSS] A. N. Kirillov, A. Schilling and M. Shimozono, A bijection between Littlewood-Richardson tableaux and rigged configurations, math.CO/9901037.

[Kl] M. Kleber, Combinatorial structure of finite-dimensional representations of Yangians: the simply-laced case, Int. Math. Res. Not. 7 (1997) 187-201.

[KT] K.Koike, I.Terada, Young-Diagrammatic Methods for the Representation Theory of the Classical Groups of Type $B_{n}, C_{n}, D_{n}$, J. of Alg. 107 (1987) $466-511$.

$[\mathrm{Ku}]$ A. Kuniba, Thermodynamics of $U_{q}\left(X_{r}^{(1)}\right)$ Bethe ansatz system with $q$ a root of unity, Nucl. Phys. B389 (1993) 209-244.

[KMOTU] A. Kuniba, K. C. Misra, M. Okado, T. Takagi and J. Uchiyama, Characters of Demazure modules and solvable lattice models, Nucl. Phys. B510 [PM] (1998) 555-576.

$[\mathrm{KN}]$ A. Kuniba and T. Nakanishi, Bethe equation at $q=0$, Möbius inversion formula, and weight multiplicities: II. $X_{n}$ case, math.QA/0008047, J. of Alg. in press.

[KNS] A. Kuniba, T. Nakanishi and J. Suzuki, Characters in conformal field theories from thermodynamic Bethe ansatz, Mod. Phys. Lett. A8 (1993) $1649-1659$.

[KNT] A. Kuniba, T. Nakanishi, Z. Tsuboi, in preparation. 
[KS] A. Kuniba, J. Suzuki, Functional relations and analytic Bethe ansatz for twisted quantum affine algebras, J. Phys. . A28 (1995) 711-722.

[LLT] A. Lascoux, B. Leclerc, J.-Y. Thibon, Ribbon Tableaux, Hall-Littlewood Functions, Quantum Affine Algebras and Unipotent Varieties, J. Math. Phys. 38 (1997) 1041-1068.

[LT] B. Leclerc and J.-Y. Thibon, Littlewood-Richardson coefficients and Kazhdan-Lusztig polynomials, Adv. Stud. in Pure Math. 28 (2000) 155220 .

[LP] J. Lepowsky and M. Primc, Structure of the standard modules for the affine Lie algebra $A_{n}^{(1)}$, Contemporary Math. 46 AMS, Providence (1985).

[L] D. E. Littlewood, The theory of group characters and matrix representations of groups, 2nd edition, Oxford Univ. Press, Oxford (1950).

[Lu] G. Lusztig, Fermionic form and Betti numbers, math.QA/0005010.

[M] B.M. McCoy, Quasi-particles and the generalized Rogers-Ramanujan identities, XIIth International Congress of Mathematical Physics (ICMP '97) (Brisbane), Internat. Press, Cambridge, MA (1999) 350-356.

[Me] E. Melzer, Fermionic character sums and the corner transfer matrix, Int. J. Mod. Phys. A9 (1994) 1115-1136.

$[\mathrm{N}]$ H. Nakajima, t-analogue of the q-characters of finite dimensional representations of quantum affine algebras, math.QA/0009231.

[NY1] A. Nakayashiki and Y. Yamada, Kostka polynomials and energy functions in solvable lattice models, Selecta Mathematica, New Ser. 3 (1997) 547-599.

[NY2] A. Nakayashiki and Y. Yamada, On spinon character formulas, in "Frontiers in Quantum Field Theory" (Itoyama et. al. ed. World Scientific, 1996) $367-371$.

[NY3] A. Nakayashiki and Y. Yamada, Crystallizing the spinon basis, Commun. Math. Phys. 178 (1996) 179-200.

[NY4] A. Nakayashiki and Y. Yamada, Crystalline spinon basis for RSOS models, Int. J. Mod. Phys. A11 (1996) 395-408.

[OPW] D. L. O'Brien, P. A. Pearce and S. O. Warnaar, Analytic calculation of conformal partition functions: Tricritical hard squares with fixed boundaries, Nucl. Phys. B501 [FS] (1997) 773-799.

[OW] E. Ogievetsky and P. Wiegmann, Factorized S-matrix and the Bethe ansatz for simple Lie groups, Phys. Lett. B168 (1986) 360-366.

[RS] B. Richmond and G. Szekeres, Some formulas related to dilogarithms, the zeta function and the Andrews-Gordon identities, J. Austral. Math. Soc. (Series A) 31 (1981) 362-373. 
[RW] N. Yu. Reshetikhin and P. B. Wiegmann, Towards the classification of completely integrable quantum field theories (The Bethe-ansatz associated with Dynkin diagrams and their automorphisms), Phys. Lett. B189 (1987) $125-131$.

[Sc] A. Schilling, Polynomial Fermionic Forms for the Branching Functions of the Rational Coset Conformal Field Theories $\widehat{s u}(2)_{M} \times \widehat{s u}(2)_{N} / \widehat{s u}(2)_{M+N}$ Nucl. Phys. B459 (1996) 393-436.

[SS1] A. Schilling and M. Shimozono, Fermionic formulas for levelrestricted generalized Kostka polynomials and coset branching functions, math.QA/0001114.

[SS2] A. Schilling and M. Shimozono, Bosonic formula for level-restricted paths, Adv. Stud. in Pure Math. 28 (2000) 305-325.

[SW] A. Schilling and S. O. Warnaar, Inhomogeneous lattice paths, generalized Kostka polynomials and $A_{n-1}$ supernomials, Commun. Math. Phys. 202 (1999) 359-401.

[S] M. Shimozono, Affine type A crystal structure on tensor products of rectangles, Demazure characters, and nilpotent varieties, math.QA/9804039.

[Ta] K. Takemura, The decomposition of level-1 irreducible highest-weight modules with respect to the level-0 actions of the quantum affine algebra, J. Phys. A31 (1998) 1467-1485.

[T] Z. Tsuboi, Analytic Bethe ansatz and functional relations related to tensorlike representations of type-II Lie superalgebras $B(r \mid s)$ and $D(r \mid s)$, J. Phys.. A32 (1999) 7175-7206.

[Wa] S. O. Warnaar, Fermionic solution of the Andrews-Baxter-Forrester model I: unification of TBA and CTM methods, J. Stat. Phys. 82 (1996) 657-685.

[W] M. L. Whippman, Branching Rules for Simple Lie Groups, J. Math. Phys. 6 (1965) 1534-1539.

[Wy] B. G. Wybourne Internal labeling and the group $G_{2}$, J. Math. Phys. 13 (1972) 455-457. 